\title{
THE GAUGE GROUP OF A NONCOMMUTATIVE PRINCIPAL BUNDLE AND TWIST DEFORMATIONS
}

\author{
PAOLO ASCHIERI, GIOVANNI LANDI, CHIARA PAGANI
}

\begin{abstract}
We study noncommutative principal bundles (Hopf-Galois extensions) in the context of coquasitriangular Hopf algebras and their monoidal category of comodule algebras. When the total space is quasi-commutative, and thus the base space subalgebra is central, we define the gauge group as the group of vertical automorphisms or equivalently as the group of equivariant algebra maps. We study Drinfeld twist (2-cocycle) deformations of Hopf-Galois extensions and show that the gauge group of the twisted extension is isomorphic to the gauge group of the initial extension. In particular, noncommutative principal bundles arising via twist deformation of commutative principal bundles have classical gauge group. We illustrate the theory with a few examples.
\end{abstract}

\section{Contents}

1. Introduction

1.1. Background material

2. Hopf-Galois extensions for coquasitriangular Hopf algebras

2.1. Hopf-Galois extensions

2.2. Coquasitriangular Hopf algebras

2.3. Hopf-Galois extensions for coquasitriangular Hopf algebras

3. The gauge group

3.1. The gauge group of equivariant algebra maps

3.2. The gauge group of vertical automorphisms

3.3. Equivalence of the gauge groups

4. Deformations by 2-cocycles

4.1. Twisting comodule algebras and coalgebras by 2-cocycles

4.2. The coquasitriangular case

4.3. Twisting Hopf-Galois extensions

4.4. Twisting gauge groups

4.5. Tensoring Hopf-Galois extensions

Appendix A. The canonical map as a morphism of relative Hopf modules

References

\section{INTRODUCTION}

Noncommutative gauge theories have emerged in different contexts in mathematics and physics. The present study aims at a better understanding of the geometric structures underlying these theories. The relevant framework is that of noncommutative principal bundles that we approach from the algebraic perspective of Hopf-Galois extensions. These first emerged as a generalization of classical Galois field extensions and were later recognised to be suitable for a description of principality of actions in algebraic and noncommutative geometry. Aiming at the noncommutative differential geometry of HopfGalois extensions, with a theory of connections and their moduli spaces, in this paper we 
study the notion of group of noncommutative gauge transformations.

For Hopf-Galois extensions the group of gauge transformations was considered in [4] and further studied in [3], (see also [9]). An unusual feature of these works is that the group there defined is bigger than one would expect. Classically, the group of gauge transformations of a principal $G$-bundle $P \rightarrow M$ is the group of vertical bundle automorphisms $P \rightarrow P$ or of $G$-equivariant maps $P \rightarrow G$, for the adjoint action of $G$ onto itself. The pull-back of these maps to the algebra of functions gives $O(G)$-equivariant algebra maps $\mathcal{O}(G) \rightarrow \mathcal{O}(P)$. However, with the definition of these papers, for the Hopf-Galois extension $\mathcal{O}(P)$ one would get the bigger group of $O(G)$-equivariant unital and convolution invertible linear maps $\mathcal{O}(G) \rightarrow \mathcal{O}(P)$, which are not necessarily algebra maps. This suggests for gauge transformations to retain some algebra map property.

As a way of clarification, let us consider the simplest case of the bundle $G \rightarrow\{*\}$ over a point. This is an elementary example of a Galois object, that is a Hopf-Galois extension of the ground field [19, Def.7.11]. Then, gauge transformations, as $O(G)$-equivariant algebra maps $\mathcal{O}(G) \rightarrow \mathcal{O}(G)$, are a copy of $G$ itself. Thus they make a much smaller group than that of all $O(G)$-equivariant unital and convolution invertible linear maps $\mathcal{O}(G) \rightarrow \mathcal{O}(G)$. Similarly, infinitesimal gauge transformations are left invariant vector fields, giving then the Lie algebra $\mathfrak{g}$ of $G$. In the dual picture they act on $\mathcal{O}(G)$ as derivations, that is, as infinitesimal algebra maps. Without requiring infinitesimal automorphisms of $\mathcal{O}(G)$ to be derivations, one obtains the whole universal enveloping algebra $U(\mathfrak{g})$. With quantum groups one can consider their universal enveloping algebra, or construct a quantum Lie algebra of left invariant vector fields that are deformed derivations (à la Woronowicz [31]).

Quantum Lie algebras have been used for infinitesimal gauge transformations for gauge field theories for example in [5, 6]. A further independent argument in favour of a theory of noncommutative gauge groups that does not drastically depart from the classical one comes from the Seiberg-Witten map between commutative and noncommutative gauge theories [30]. This map (initially considered for noncommutative gauge theories in the context of string theory and related flux compactification) establishes a one-to-one correspondence between commutative and noncommutative gauge transformations and hence points to a noncommutative gauge group that is a deformation of the classical one.

Other studies suggesting a view on gauge transformations as (deformed) algebra maps are those on noncommutative instanton moduli spaces, for example [22] for instantons on the principal bundle on the noncommutative four sphere $S_{\theta}^{4}$ [21]. There the dimension of the moduli space survives the $\theta$-deformation (see also [2]).

In the present paper we study the group of gauge transformations as the group of equivariant algebra maps. By way of comparison, let us anticipate here our results for the case of the Galois object $\left(\mathcal{O}(G)_{\bullet_{\gamma}}, \mathcal{O}(G)_{\gamma}\right)$, with the quantum structure group $\mathcal{O}(G)_{\gamma}$ coacting on the total space algebra $\mathcal{O}(G)_{\bullet_{\gamma}}$ (in general this is not a trivial Hopf-Galois extension, but only a cleft one). We change the multiplication in $\mathcal{O}(G)_{\gamma}$ by considering the braided Hopf algebra $\mathcal{O}(G)_{\gamma}$ so that we find gauge transformations as $\mathcal{O}(G)_{\gamma}$-equivariant algebra maps $\mathcal{O}(G)_{\gamma} \overrightarrow{\rightarrow \mathcal{O}(G)} \bullet_{\bullet_{\gamma}}$. These are deformed algebra maps with respect to the initial product in $\mathcal{O}(G)_{\gamma}$.

In [1] noncommutative principal bundles were revisited and considered in a categorical perspective. Since noncommutative principal bundles with Hopf algebra (quantum structure group) $H$ are $H$-comodule algebras $A$ with a canonically given $H$-equivariant map $\chi$, required to be invertible, the basic category where to study these objects is that of $H$-(co)representations, that is that of $H$-comodules. A second category where they 
can be studied is that of $H$ and $K$-comodules, with $K$ an "external Hopf algebra of symmetries", a Hopf algebra associated with the automorphisms of a Hopf-Galois extension (classically a group acting via equivariant maps differing from the identity on the base space). In [1] Hopf Galois extensions were studied in these two categorical settings and it was shown that Drinfeld twists (Hopf algebra 2-cocycles) deform functorially Hopf-Galois extensions to Hopf-Galois extensions. Considering a twist on the Hopf algebra $H$ leads to a deformation of the fibers of the principal bundle; considering a twist on the external symmetry Hopf algebra $K$ leads to a deformation of the base space. Combining twists on $H$ and on $K$ one obtains deformations of both the fibers and the base space. Many examples were provided starting from commutative principal bundles.

In the present paper we work within the representation category of an Hopf algebra $H$, with $A$ an $H$-comodule algebra. We study gauge transformations of noncommutative principal bundles $B=A^{c o H} \subseteq A$ with quantum structure group $H$, noncommutative total space $A$ and commutative base $B$. Examples motivating the interest in this case include also quantum group gauge theory on lattices, that is related to models quantizing the algebra of observables of Chern-Simons theory [25].

In a sequel paper we consider Hopf algebras $H$ and Hopf-Galois extensions in a category of $K$-comodules. In this richer context we study gauge transformations of Hopf-Galois extensions with noncommutative bases (for instance noncommutative tori and related manifolds). A further motivation for these studies comes from the relevance of noncommutative gauge field theories for string theory and related compactifications. There $U(N)$ gauge theories on noncommutative tori naturally emerge [7]. In that context already considering simple Lie groups (like $S U(N)$ or $S O(N)$ ) is problematic, one way out being the use of the Seiberg-Witten map between commutative and noncommutative gauge theories [30, 18], another approch possibly being the Hopf-Galois one we are pursuing.

Before considering gauge transformations as algebra maps, we study conditions for the canonical map $\chi$ to be an algebra map. The natural categorical setting for addressing this question is that of coquasitriangular Hopf algebras. Indeed in this context the category of $H$-comodule algebras is a monoidal category. We show that the canonical map is a morphism in the category when the multiplication in $A$ is a morphism as well (we call such comodule algebras quasi-commutative). This implies that the base $B$ is commutative. Canonically associated with a coquasitriangular Hopf algebra $H$ we have the braided Hopf algebra $\underline{H}$. The gauge group is first defined as the set of $H$-equivariant (unital) algebra maps $\underline{H} \rightarrow A$ and then proven to be a group. A second approach is to define the gauge group as the set of $H$-equivariant algebra maps $A \rightarrow A$ that restrict to the identity on $B$. This corresponds to the classical picture of vertical authomorphisms of a principal bundle. Here too we prove that these maps form a group. These two definitions of gauge group are then shown to be equivalent, and the theory is illustrated with examples.

We study next Drinfeld twist deformations of Hopf-Galois extensions and of their gauge groups. We refine the results in [1] to the case of coquasitriangular and cotriangular Hopf algebras. A twist on $H$ induces an equivalence of the associated monoidal categories, and braided Hopf algebras are twisted to braided Hopf algebras. The equivalence of the possible different twisting procedures is proven via a map $\mathcal{Q}$. This map is related to the natural isomorphism that gives the equivalence of the categories of Hopf algebra modules and of twisted Hopf algebra modules as closed monoidal categories. These results allow us to conclude that Hopf-Galois extensions $B=A^{c o H} \subseteq A$, with canonical map $\chi$ that is 
an algebra map, are twisted to Hopf-Galois extensions $B=A_{\gamma}^{\mathrm{coH}_{\gamma}} \subseteq A_{\gamma}$ with canonical map $\chi_{\gamma}$ that is an algebra map. The twist functor is then applied to the two equivalent characterizations of the gauge group of a Hopf-Galois extension. By using again the map $\mathcal{Q}$ we show that the initial gauge group and the twisted one are isomophic. In particular cleft (but not necessarily trivial) Hopf-Galois extensions obtained twisting trivial HopfGalois extensions have isomorphic gauge groups.

Finally, we consider tensor products of noncommutative principal bundles and study the resulting gauge groups. Combining the tensor product construction and the twisting procedure we construct interesting examples. In particular we study the noncommutative principal fibration of spheres $S^{7} \times_{\gamma} S^{1} \rightarrow S^{4}$ on the commutative 4-sphere. The structure group is $U_{q}(2)$, a cotriangular deformation of the unitary group, and the gauge group of this Hopf-Galois extension is isomorphic to the direct product of the classical gauge group of the instanton bundle on the 4-sphere $S^{4}$ with the group of $U(1)$-valued functions on $S^{4}$.

We mention that the idea of using a braiding that renders the Hopf-Galois canonical map an algebra homomorphism, so as to enable one to define gauge transformations, was already put forward in [13. There it is shown that the algebra structure on the left-hand side of the canonical map, that is induced from the tensor algebra on its right-hand side, is given by a braiding of Hopf algebras. Then, for cosemisimple commutative Hopf algebras this braiding was used to define gauge transformations as algebra homomorphisms. While the braiding in [13] is written using the inverse of the canonical map, in the present paper the braiding comes from the coquasitriangular structure of the class of Hopf algebras considered.

\subsection{Background material.}

We work in the category of $\mathbb{K}$-modules, for $\mathbb{K}$ a fixed commutative field with unit $1_{\mathbb{K}}$ or the ring of formal power series in a variable $\hbar$ over a field. Much of what follows can be generalised to $\mathbb{K}$ a commutative unital ring. We denote simply by $\otimes$ the tensor product over $\mathbb{K}$. All algebras will be over $\mathbb{K}$ and assumed to be unital and associative. The product in an algebra $A$ is denoted by $m_{A}: A \otimes A \rightarrow A, a \otimes b \mapsto a b$ and the unit map by $\eta_{A}: \mathbb{K} \rightarrow A$, with $1_{A}:=\eta_{A}\left(1_{\mathbb{K}}\right)$ the unit element. Morphisms of algebras will be assumed to be unital. Analogously all coalgebras will be over $\mathbb{K}$ and assumed to be counital and coassociative. The coproduct and counit of a coalgebra $C$ are denoted by $\Delta_{C}: C \rightarrow C \otimes C$ and $\varepsilon_{C}: C \rightarrow \mathbb{K}$ respectively. We use the standard Sweedler notation for the coproduct: $\Delta_{C}(c)=c_{(1)} \otimes c_{(2)}$ (sum understood), for all $c \in C$, and for its iterations: $\Delta_{C}^{n}=\left(\mathrm{id} \otimes \Delta_{C}\right) \circ \Delta_{C}^{n-1}: c \mapsto c_{(1)} \otimes c_{(2)} \otimes \cdots \otimes c_{(n+1)}, n>1$. We denote by $*$ the convolution product in the dual $\mathbb{K}$-module $C^{\prime}:=\operatorname{Hom}(C, \mathbb{K}),(f * g)(c):=f\left(c_{(1)}\right) g\left(c_{(2)}\right)$, for all $c \in C$, $f, g \in C^{\prime}$. For a Hopf algebra $H$, we denote by $S_{H}: H \rightarrow H$ its antipode. For all these maps we will omit the subscripts which refer to the co/algebras involved when no risk of confusion can occur. We simply write $V \in \mathcal{C}$ for an object $V$ in a category $\mathcal{C}$, and $\operatorname{Hom}_{\mathcal{C}}(-,-)$ for morphisms between any two objects. Finally, all monoidal categories in this paper will have a trivial associator, hence we can unambiguously write $V_{1} \otimes V_{2} \otimes \cdots \otimes V_{n}$ for the tensor product of $n$ objects.

Given a bialgebra (or a Hopf algebra) $H$, we denote by $\mathcal{M}^{H}$ the category of right $H$ comodules: a right $H$-comodule is a $\mathbb{K}$-module $V$ with a $\mathbb{K}$-linear map $\delta^{V}: V \rightarrow V \otimes H$ (a right $H$-coaction) such that

$$
(\mathrm{id} \otimes \Delta) \circ \delta^{V}=\left(\delta^{V} \otimes \mathrm{id}\right) \circ \delta^{V}, \quad(\mathrm{id} \otimes \varepsilon) \circ \delta^{V}=\mathrm{id} .
$$


In Sweedler notation we write $\delta^{V}: V \rightarrow V \otimes H, v \mapsto \delta^{V}=v_{(0)} \otimes v_{(1)}$, and the right $H$-comodule properties (1.1) read, for all $v \in V$,

$v_{(0)} \otimes\left(v_{(1)}\right)_{(1)} \otimes\left(v_{(1)}\right)_{(2)}=\left(v_{(0)}\right)_{(0)} \otimes\left(v_{(0)}\right)_{(1)} \otimes v_{(1)}=: v_{(0)} \otimes v_{(1)} \otimes v_{(2)}, \quad v_{(0)} \varepsilon\left(v_{(1)}\right)=v$.

A morphism between $V, W \in \mathcal{M}^{H}$ is a $\mathbb{K}$-linear map $\psi: V \rightarrow W$ which is $H$-equivariant: $\delta^{W} \circ \psi=(\psi \otimes \mathrm{id}) \circ \delta^{V}$. We equivalently say that $\psi: V \rightarrow W$ is an $H$-comodule map.

In fact, $\mathcal{M}^{H}$ is a monoidal category: given $V, W \in \mathcal{M}^{H}$, the tensor product $V \otimes W$ of $\mathbb{K}$-modules is an object in $\mathcal{M}^{H}$ with the right $H$-coaction

$$
\delta^{V \otimes W}: V \otimes W \longrightarrow V \otimes W \otimes H, \quad v \otimes w \longmapsto v_{(0)} \otimes w_{(0)} \otimes v_{(1)} w_{(1)} .
$$

The unit object in $\mathcal{M}^{H}$ is $\mathbb{K}$ with coaction $\delta^{\mathbb{K}}$ given by the unit map $\eta_{H}: \mathbb{K} \rightarrow \mathbb{K} \otimes H \simeq H$.

We denote by $\mathcal{A}^{H}$ the category of right $H$-comodule algebras: a right $H$-comodule algebra is an algebra $A$ which is a right $H$-comodule such that the multiplication and unit of $A$ are morphisms of $H$-comodules. This is equivalent to requiring the coaction $\delta^{A}: A \rightarrow A \otimes H$ to be a morphism of unital algebras (where $A \otimes H$ has the usual tensor product algebra structure): for all $a, a^{\prime} \in A$,

$$
\delta^{A}\left(a a^{\prime}\right)=\delta^{A}(a) \delta^{A}\left(a^{\prime}\right) \quad, \quad \delta^{A}\left(1_{A}\right)=1_{A} \otimes 1_{H} .
$$

Morphisms in $\mathcal{A}^{H}$ are $H$-comodule maps which are also algebra maps.

We denote by $\mathcal{C}^{H}$ the category of right $H$-comodule coalgebras: a right $H$-comodule coalgebra is a coalgebra $C$ which is a right $H$-comodule and such that the coproduct and the counit are morphisms of $H$-comodules that is, for each $c \in C$

$$
\left(c_{(1)}\right)_{(0)} \otimes\left(c_{(2)}\right)_{(0)} \otimes\left(c_{(1)}\right)_{(1)}\left(c_{(2)}\right)_{(1)}=\left(c_{(0)}\right)_{(1)} \otimes\left(c_{(0)}\right)_{(2)} \otimes c_{(1)}, \quad \varepsilon\left(c_{(0)}\right) c_{(1)}=\varepsilon(c) 1_{H} .
$$

Morphisms in $\mathcal{C}^{H}$ are $H$-comodule maps which are also coalgebra maps.

Let $H$ be a bialgebra and let $A \in \mathcal{A}^{H}$. An $(A, H)$-relative Hopf module $V$ is a right $H$-comodule with a compatible left $A$-module structure, that is the left $A$-action $\triangleright_{V}$ is a morphism of $H$-comodules such that the following diagram commutes

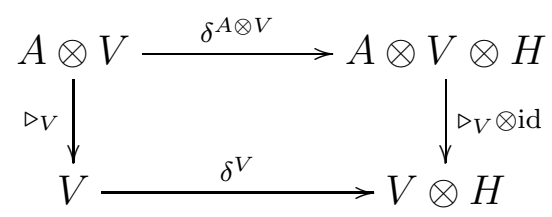

Explicitly, for all $a \in A$ and $v \in V$,

$$
\left(a \triangleright_{V} v\right)_{(0)} \otimes\left(a \triangleright_{V} v\right)_{(1)}=a_{(0)} \triangleright_{V} v_{(0)} \otimes a_{(1)} v_{(1)} .
$$

A morphism of $(A, H)$-relative Hopf modules is a morphism of right $H$-comodules which is also an $A$-linear map, that is a morphism of left $A$-modules. We denote by ${ }_{A} \mathcal{M}^{H}$ the category of $(A, H)$-relative Hopf modules. In a similar way one defines the categories of relative Hopf modules $\mathcal{M}_{A}{ }^{H}$ for $A$ acting on the right, and ${ }_{E} \mathcal{M}_{A}{ }^{H}$ for right $A$ and left $E$ compatible actions, with $E \in \mathcal{A}^{H}$.

\section{Hopf-Galois extensions for COQuasitriangular Hopf Algebras}

We consider noncommutative principal bundles as Hopf-Galois extensions. These are $H$-comodule algebras $A$ with a canonically defined map $\chi: A \otimes_{B} A \rightarrow A \otimes H$ which is required to be invertible. We first consider the category of $(A, H)$-relative Hopf modules and understand within this monoidal category the notion of Hopf-Galois extension, that is the bijectivity of the map $\chi$. This is done in $\S 2.1$, where we see that the monoidal structure forces $H$ in $A \otimes H$ to be considered as an $H$-comodule with the adjoint action Ad, denoted $\underline{H}$. In $\S 2.2$ we consider the case of $H$ coquasitriangular, where the category 
of $H$-comodule algebras is monoidal. The braided Hopf algebra $\underline{H}$ with the adjoint action Ad is an $H$-comodule algebra so that both $A \otimes A$ and $A \otimes H$ are $H$-comodule algebras. The canonical map is then proven to be an algebra map provided $A$ is quasi-commutative.

\subsection{Hopf-Galois extensions.}

Definition 2.1. Let $H$ be a Hopf algebra and let $A \in \mathcal{A}^{H}$ with coaction $\delta^{A}$. Consider the subalgebra $B:=A^{c o H}=\left\{b \in A \mid \delta^{A}(b)=b \otimes 1_{H}\right\} \subseteq A$ of coinvariant elements (elements invariant under the $H$-coaction) and let $A \otimes_{B} A:=A \otimes A /\left\langle a \otimes b a^{\prime}-a b \otimes a^{\prime}\right\rangle_{a, a^{\prime} \in A, b \in B}$ be the corresponding balanced tensor product. The extension $B \subseteq A$ is called an $H$-HopfGalois extension provided the (so-called) canonical map

$$
\chi:=(m \otimes \mathrm{id}) \circ\left(\mathrm{id} \otimes_{B} \delta^{A}\right): A \otimes_{B} A \longrightarrow A \otimes H, \quad a^{\prime} \otimes_{B} a \longmapsto a^{\prime} a_{(0)} \otimes a_{(1)}
$$

is bijective.

The canonical map $\chi$ is a morphism in the category ${ }_{A} \mathcal{M}_{A}{ }^{H}$ of relative Hopf modules [1. Both $A \otimes_{B} A$ and $A \otimes H$ are objects in ${ }_{A} \mathcal{M}_{A}{ }^{H}$. The left $A$-module structures are given by the left multiplication on the first factors while the right $A$-actions are given by

$$
\left(a \otimes_{B} a^{\prime}\right) a^{\prime \prime}:=a \otimes_{B} a^{\prime} a^{\prime \prime} \quad \text { and } \quad(a \otimes h) a^{\prime}:=a a_{(0)}^{\prime} \otimes h a_{(1)}^{\prime} .
$$

As for the $H$-comodule structure, the tensor product $A \otimes A$ has the natural right $H$ coaction induced by the monoidal structure of $\mathcal{M}^{H}$, as in (1.2),

$$
\delta^{A \otimes A}: A \otimes A \rightarrow A \otimes A \otimes H, \quad a \otimes a^{\prime} \mapsto a_{(0)} \otimes a_{(0)}^{\prime} \otimes a_{(1)} a_{(1)}^{\prime}
$$

for all $a, a^{\prime} \in A$. This descends to the quotient $A \otimes_{B} A$ because $B \subseteq A$ is the subalgebra of $H$-coinvariants. Similarly, $A \otimes H$ is endowed with the tensor product coaction, where we regard the Hopf algebra $H$ as a right $H$-comodule with the right adjoint $H$-coaction

$$
\text { Ad }: h \longmapsto h_{(2)} \otimes S\left(h_{(1)}\right) h_{(3)} \text {. }
$$

The right $H$-coaction on $A \otimes H$ is then given again as in (1.2) by

$$
\delta^{A \otimes H}(a \otimes h)=a_{(0)} \otimes h_{(2)} \otimes a_{(1)} S\left(h_{(1)}\right) h_{(3)} \in A \otimes H \otimes H
$$

for all $a \in A, h \in H$. Both $A \otimes_{B} A$ and $A \otimes H$ are shown to be objects in ${ }_{A} \mathcal{M}_{A}{ }^{H}$ with respect to these structures and $\chi$ to be a morphism in the category ${ }_{A} \mathcal{M}_{A}{ }^{H}$ of relative Hopf modules (see [1] for details, and see Appendix A for a comparison with other descriptions of the map $\chi$ as a morphism of relative Hopf modules).

Since the canonical map $\chi$ is left $A$-linear, its inverse is determined by the restriction $\tau:=\chi_{\left.\right|_{1 \otimes H}}^{-1}$, named translation map,

$$
\tau=\left.\chi^{-1}\right|_{1 \otimes H}: H \rightarrow A \otimes_{B} A, \quad h \mapsto h^{<1>} \otimes_{B} h^{<2>} .
$$

We recall for later use the following properties of the translation map (see Appendix A.1).

$$
\left(\mathrm{id} \otimes_{B} \delta^{A}\right) \circ \tau=(\tau \otimes \mathrm{id}) \Delta \quad, \quad(\tau \otimes S) \circ \text { flip } \circ \Delta=(\mathrm{id} \otimes \text { flip }) \circ\left(\delta^{A} \otimes_{B} \mathrm{id}\right) \circ \tau,
$$

that on the generic element $h \in H$ respectively read

$$
\begin{gathered}
h^{<1>} \otimes_{B} h^{<2>}{ }_{(0)} \otimes h^{<2>}{ }_{(1)}=h_{(1)}{ }^{<1>} \otimes_{B} h_{(1)}^{<2>} \otimes h_{(2)}, \\
h_{(2)}{ }^{<1>} \otimes_{B} h_{(2)}^{<2>} \otimes S\left(h_{(1)}\right)=h^{<1>}{ }_{(0)} \otimes_{B} h^{<2>} \otimes h^{<1>}{ }_{(1)} .
\end{gathered}
$$

A Hopf-Galois extension is cleft if there exists a convolution invertible morphism of $H$ comodules $j: H \rightarrow A$ (the cleaving map), where $H$ has coaction $\Delta$. This is equivalent to an isomorphism $A \simeq B \otimes H$ of left $B$-modules and right $H$-comodules, where $B \otimes H$ is a left $B$-module via multiplication on the left and a right $\mathrm{H}$-comodule via id $\otimes \Delta$. A Hopf-Galois extension is a trivial extension if the cleaving map is also an algebra map. 
Commutative Hopf-Galois extensions typically arise when considering principal $G$ bundles. Twisted versions will be described in Section 4.3 below.

Example 2.2. Let $G$ be a semisimple affine algebraic group and let $\pi: P \rightarrow P / G$ be a principal $G$-bundle with $P$ and $P / G$ affine varieties. Let $H=\mathcal{O}(G)$ be the dual coordinate Hopf algebra and $A=\mathcal{O}(P), B=\mathcal{O}(P / G)$ the corresponding coordinate algebras. Let $B \subseteq A$ be the subalgebra of functions constant on the fibers, we then have $B=A^{c o H}$ and $\mathcal{O}\left(P \times_{P / G} P\right) \simeq A \otimes_{B} A$. The bijectivity of the map $P \times G \rightarrow P \times_{P / G} P,(p, g) \mapsto(p, p g)$, characterizing principal bundles in this context, corresponds to the bijectivity of the canonical map $\chi: A \otimes_{B} A \rightarrow A \otimes H$, thus showing that $B=A^{c o H} \subseteq A$ is a HopfGalois extension (see e.g. [26, §8.5] and [10, Thm.3.1.5]). An important notion is that of the classical translation map $t: P \times_{P / G} P \rightarrow G,(p, q) \mapsto t(p, q)$ where $q=p t(p, q)$. Properties (2.5) and (2.6) then read: $t(p, q g)=t(p, q) g$ and $t(p g, q)=g^{-1} t(p, q)$.

\subsection{Coquasitriangular Hopf algebras.}

We begin by recalling basic properties of coquasitriangular Hopf algebras; for proofs we refer e.g. to [20, Ch.10] or [24, Ch.2]. We then study the monoidal category of comodule algebras $\left(\mathcal{A}^{H}, \otimes\right)$ and the braided Hopf algebra $\underline{H} \in \mathcal{A}^{H}$.

Definition 2.3. A bialgebra $H$ is called coquasitriangular (or dual quasitriangular) if it is endowed with a linear form $R: H \otimes H \rightarrow \mathbb{K}$ such that

(i) $R$ is invertible for the convolution product, with inverse denoted by $\bar{R}$;

(ii) $m_{o p}=R * m * \bar{R}$, that is, for all $h, k \in H$,

$$
k h=R\left(h_{(1)} \otimes k_{(1)}\right) h_{(2)} k_{(2)} \bar{R}\left(h_{(3)} \otimes k_{(3)}\right) ;
$$

(iii) $R \circ(m \otimes \mathrm{id})=R_{13} * R_{23} \quad$ and $\quad R \circ(\mathrm{id} \otimes m)=R_{13} * R_{12}$, where $R_{12}(h \otimes k \otimes l)=R(h \otimes k) \varepsilon(l)$ and similarly for $R_{13}$ and $R_{23}$; in components, for all $h, k, l \in H$, these conditions read

$$
R(h k \otimes l)=R\left(h \otimes l_{(1)}\right) R\left(k \otimes l_{(2)}\right) \quad \text { and } \quad R(h \otimes k l)=R\left(h_{(2)} \otimes k\right) R\left(h_{(1)} \otimes l\right) .
$$

The linear form $R$ is called a universal $R$-form of $H$. If $(H, R)$ is coquasitriangular then so is $\left(H, \bar{R}_{21}\right)$ where $R_{21}(h \otimes k):=R(k \otimes h)$ for all $h, k \in H$. A coquasitriangular bialgebra $(H, R)$ is called cotriangular if $R=\bar{R}_{21}$.

A Hopf algebra $H$ is called co(quasi)triangular if it is such as a bialgebra.

Example 2.4. Any commutative bialgebra $H$ is cotriangular with (trivial) universal $R$ form $R=\varepsilon \otimes \varepsilon$.

Note that if a coquasitriangular bialgebra $(H, R)$ is cocommutative, then it is commutative. Nonetheless, this does not imply that $R$ is trivial:

Example 2.5. Let $H=\mathbb{C} \mathbb{Z}$ be the group Hopf-algebra of the abelian group $\mathbb{Z}$. It is the commutative and cocommutative Hopf algebra generated by an invertible element $g$, $\mathbb{C Z}=\mathbb{C}\left[g, g^{-1}\right]$, with $\Delta(g)=g \otimes g, \varepsilon(g)=1, S(g)=g^{-1}$. For every complex number $q \neq 1$, this Hopf algebra is coquasitriangular with $R$-form $R_{q}\left(g^{n}, g^{m}\right)=q^{-n m}$.

Example 2.6. The FRT bialgebras $\mathcal{O}\left(G_{q}\right)$, noncommutative deformations of the coordinate algebra on the Lie groups $G$ of the $A, B, C, D$ series, are coquasitriangular [16].

Example 2.7. If $(H, R)$ is a coquasitriangular Hopf algebra and $\gamma: H \otimes H \rightarrow \mathbb{K}$ is a 2-cocycle on $H$, then the Hopf algebra $H_{\gamma}$ with twisted product and antipode (see 44.1 ) is also coquasitriangular with universal $R$-form

$$
R_{\gamma}:=\gamma_{21} * R * \bar{\gamma}: h \otimes k \longmapsto \gamma\left(k_{(1)} \otimes h_{(1)}\right) R\left(h_{(2)} \otimes k_{(2)}\right) \bar{\gamma}\left(h_{(3)} \otimes k_{(3)}\right)
$$


where $\bar{\gamma}: H \otimes H \rightarrow \mathbb{K}$ is the convolution inverse of $\gamma$. The (convolution) inverse of $R$ is $\bar{R}_{\gamma}:=\gamma * \bar{R} * \bar{\gamma}_{21}$. If $(H, R)$ is cotriangular, then $\left(H_{\gamma}, R_{\gamma}\right)$ is cotriangular.

From its definition, it follows that the $R$-form of a coquasitriangular bialgebra $(H, R)$ is normalized, that is, for all $h \in H$,

$$
R(1 \otimes h)=\varepsilon(h)=R(h \otimes 1),
$$

and that it satisfies the Yang-Baxter-Equation $R_{12} * R_{13} * R_{23}=R_{23} * R_{13} * R_{12}$, that is, for all $h, k, l \in H$,

$$
R\left(h_{(1)} \otimes k_{(1)}\right) R\left(h_{(2)} \otimes l_{(1)}\right) R\left(k_{(2)} \otimes l_{(2)}\right)=R\left(k_{(1)} \otimes l_{(1)}\right) R\left(h_{(1)} \otimes l_{(2)}\right) R\left(h_{(2)} \otimes k_{(2)}\right) .
$$

If in addition $H$ is a Hopf algebra, then for all $h, k \in H$ we have

$$
R(S(h) \otimes k)=\bar{R}(h \otimes k) ; \quad \bar{R}(h \otimes S(k))=R(h \otimes k),
$$

from which it also follows $R(S(h) \otimes S(k))=R(h \otimes k)$. Furthermore, the antipode $S$ of $H$ is invertible with inverse $S^{-1}=u_{R} * S * \bar{u}_{R}$, where

$$
u_{R}: h \longmapsto R\left(h_{(1)} \otimes S\left(h_{(2)}\right)\right) \quad ; \quad \bar{u}_{R}: h \longmapsto \bar{R}\left(S\left(h_{(1)}\right) \otimes h_{(2)}\right) .
$$

Finally, when $(H, R)$ is coquasitriangular, the monoidal category of right $H$-comodules $\mathcal{M}^{H}$ is braided monoidal with braiding given by the $H$-comodule isomorphisms

$$
\Psi_{V, W}^{R}: V \otimes W \longrightarrow W \otimes V, \quad v \otimes w \longmapsto w_{(0)} \otimes v_{(0)} R\left(v_{(1)} \otimes w_{(1)}\right) .
$$

We can now recall a key feature of coquasitriangular Hopf algebras: tensor products of comodule algebras are comodule algebras and tensor products of comodule algebra maps are again comodule algebra maps.

Proposition 2.8. Let $(H, R)$ be a coquasitriangular bialgebra. Let $\left(A, \delta^{A}\right),\left(C, \delta^{C}\right) \in \mathcal{A}^{H}$ be right $H$-comodule algebras. Then the $H$-comodule $A \otimes C$ (with tensor product coaction $\delta^{A \otimes C}: a \otimes c \mapsto a_{(0)} \otimes c_{(0)} \otimes a_{(1)} c_{(1)}$ as in (1.2) ) is a right $H$-comodule algebra when endowed with the product

$$
(a \otimes c) \cdot\left(a^{\prime} \otimes c^{\prime}\right):=a \Psi_{C, A}^{R}\left(c \otimes a^{\prime}\right) c^{\prime}=a a_{(0)}^{\prime} \otimes c_{(0)} c^{\prime} R\left(c_{(1)} \otimes a_{(1)}^{\prime}\right) .
$$

Moreover, when $\phi: A \rightarrow E$ and $\psi: C \rightarrow F$ are $H$-equivariant algebra maps, that is morphisms of $H$-comodule algebras, then so is the map $\phi \otimes \psi: A \otimes C \rightarrow E \otimes F$, $a \otimes c \mapsto \phi(a) \otimes \psi(c)$, where $A \otimes C$ and $E \otimes F$ are endowed with the -products in (2.15).

Proof. Associativity of the product in $A \otimes C$ is straighforward. The coaction $\delta^{A \otimes C}$ is also easily seen to be an algebra map because of (2.7), (an explicit proof can be found in [23], or in [20, Lem.31 §10.3]). The statement about morphisms follows by writing $\phi \otimes \psi=\left(\phi \otimes \mathrm{id}_{F}\right) \circ\left(\mathrm{id}_{A} \otimes \psi\right)$ and showing that $\mathrm{id}_{A} \otimes \phi$ and $\psi \otimes \mathrm{id}_{F}$ are both algebra maps (this is due to $H$-equivariance of $\phi$ and $\psi$ ).

The $H$-comodule algebra $(A \otimes C, \mathbf{-})$ is called the braided tensor product algebra of $A$ and $C$; we denote it by $A \otimes C$, and write $a \bigotimes c \in A \otimes C$ for $a \in A, c \in C$. Similarly, we denote by $\phi \otimes \psi:=\phi \otimes \psi: A \otimes C \rightarrow E \otimes F$ the $H$-equivariant algebra map resulting from the tensor product of the $H$-equivariant algebra maps $\phi: A \rightarrow E$ and $\psi: C \rightarrow F$.

Proposition 2.9. Let $(H, R)$ be a coquasitriangular bialgebra. The category $\mathcal{A}^{H}$ of $H$ comodule algebras endowed with the above defined tensor product $\otimes$ becomes a monoidal category, denoted $\left(\mathcal{A}^{H}, \bigotimes\right)$. 
Proof. Let $\left(A, \delta^{A}\right),\left(C, \delta^{C}\right),\left(E, \delta^{E}\right) \in \mathcal{A}^{H}$. If we forget the algebra structure the tensor product $\otimes$ becomes the associative tensor product of $H$-comodules of the monoidal category $\left(\mathcal{M}^{H}, \otimes\right)$, where $(A \otimes C) \otimes E \simeq A \otimes(C \otimes E)$ (as $\mathbb{K}$-modules). We only need to show that this isomorphism is compatible with the algebra structure, so that it is an isomorphism in $\mathcal{A}^{H}$. The equality

$$
((a \otimes c) \otimes e) \cdot\left(\left(a^{\prime} \otimes c^{\prime}\right) \otimes e^{\prime}\right)=(a \otimes(c \otimes e)) \cdot\left(a^{\prime} \otimes\left(c^{\prime} \otimes e^{\prime}\right)\right)
$$

follows from the explicit expression (2.15) for the product and the property (2.8) of the $R$-form. The units in $(A \otimes C) \otimes E$ and in $A \otimes(C \otimes E)$ trivially coincide. The unit object in $\left(\mathcal{A}^{H}, \bigotimes\right)$ is $\mathbb{K}$, seen as an $H$-comodule algebra (since $\delta^{\mathbb{K}}=\eta_{H}$ is an algebra map).

Remark 2.10. The braiding (2.14) of $\mathcal{M}^{H}$ defines a braiding

$$
\Psi_{A, C}^{R}: A \otimes C \longrightarrow C \otimes A, \quad a \otimes c \longmapsto c_{(0)} \otimes a_{(0)} R\left(a_{(1)} \otimes c_{(1)}\right)
$$

for the monoidal subcategory $\left(\mathcal{A}^{H}, \otimes\right)$ if and only if $R$ is cotriangular. Indeed the $H$ comodule isomorphisms (2.16) are algebra maps if and only if $R$ is cotriangular. Hence requiring the monoidal category $\left(\mathcal{A}^{H}, \nabla\right)$ to be braided with braidings $(2.16)$ is more specifically requiring it to be a symmetric monoidal category, that is $\left(\Psi_{A, C}^{R}\right)^{-1}=\Psi_{C, A}^{R}$.

An important role in the following is played by the right $H$-comodule $\underline{H}:=(H, \mathrm{Ad})$, with the right adjoint coaction Ad : $\underline{H} \rightarrow \underline{H} \otimes H, h \mapsto h_{(2)} \otimes S\left(h_{(1)}\right) h_{(3)}$ as defined in 92.1. The notation $\underline{H}$ is used when considering $H$ as an $H$-comodule rather than a Hopf algebra. If $H$ is coquasitriangular, one can endow $\underline{H}$ with a product that makes $\underline{H}$ an $H$-comodule algebra and a braided Hopf algebra (see e.g. [20, §10.3.2]):

Proposition 2.11. Let $(H, R)$ be a coquasitriangular Hopf algebra. The right $H$-comodule $\underline{H}=(H, \mathrm{Ad})$ becomes an $H$-comodule algebra when endowed with the product

$$
h \cdot k:=h_{(2)} k_{(2)} R\left(S\left(h_{(1)}\right) h_{(3)} \otimes S\left(k_{(1)}\right)\right)
$$

and unit $\eta: \mathbb{K} \rightarrow \underline{H}$ given, as linear map, by the unit $\eta_{H}$ of $H$.

Vice versa, the product in the Hopf algebra $H$ is recovered from that in $\underline{H}$ as

$$
h k=h_{(2)} \div k_{(2)} R\left(S\left(h_{(1)}\right) h_{(3)} \otimes k_{(1)}\right) .
$$

Given an $H$-comodule $V \in \mathcal{M}^{H}$, we denote by $\underline{\delta}^{V}: V \rightarrow V \otimes \underline{H}$ the coaction $\delta^{V}: V \rightarrow$ $V \otimes H$ thought as a linear map from $V$ to $V \otimes \underline{H}$. It is easy to show that $\underline{\delta}^{V}$ is an $H$-comodule map, that is, the commutativity of the diagram

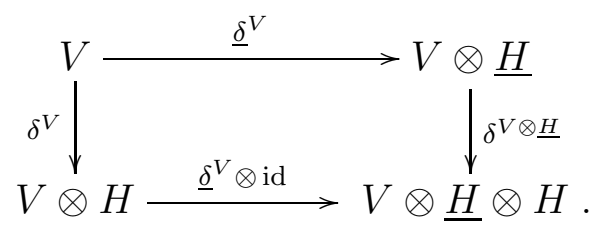

Furthermore, given an $H$-comodule algebra $A \in \mathcal{A}^{H}$, with $(H, R)$ coquasitriangular, we denote by $\underline{\delta}^{A}: A \rightarrow A \otimes \underline{H}$ the $H$-comodule map $\underline{\delta}^{A}: A \rightarrow A \otimes \underline{H}$.

Proposition 2.12. Let $(H, R)$ be a coquasitriangular Hopf algebra, $A$ an algebra in $\mathcal{M}^{H}$ with coaction $\delta^{A}: A \rightarrow A \otimes H$. The map $\underline{\delta}^{A}: A \rightarrow A \otimes \underline{H}$ is an algebra map if and only if $\delta^{A}: A \rightarrow A \otimes H$ is an algebra map. 
Proof. Let - be the product in the braided tensor product algebra $A \otimes \underline{H}$ given in (2.15). Then, for all $a, c \in A, \underline{\delta}^{A}(a) \cdot \underline{\delta}^{A}(c)=\underline{\delta}^{A}(a c) \Leftrightarrow \delta^{A}(a) \delta^{A}(c)=\delta^{A}(a c)$; indeed,

$$
\begin{aligned}
\underline{\delta}^{A}(a)-\underline{\delta}^{A}(c) & =\left(a_{(0)} \otimes a_{(1)}\right) \bullet\left(c_{(0)} \otimes c_{(1)}\right)=a_{(0)} c_{(0)} \otimes a_{(2)} \dot{-} c_{(2)} R\left(S\left(a_{(1)}\right) a_{(3)} \otimes c_{(1)}\right) \\
& =a_{(0)} c_{(0)} \otimes a_{(1)} c_{(1)}=\left(a_{(0)} \otimes a_{(1)}\right)\left(c_{(0)} \otimes c_{(1)}\right) \\
& =\delta^{A}(a) \delta^{A}(c) .
\end{aligned}
$$

Furthermore, unitality of $\underline{\delta}^{A}$ is equivalent to unitality of $\delta^{A}$ since the two maps are the same as linear maps.

Definition 2.13. Let $(H, R)$ be a coquasitriangular Hopf algebra. An $H$-comodule algebra $\left(L, m_{L}, \eta_{L}, \delta^{L}\right)$ and $H$-comodule coalgebra $\left(L, \Delta_{L}, \varepsilon_{L}, \delta^{L}\right)$ is called a braided bialgebra associated with $H$ if it is a bialgebra in the braided monoidal category $\left(\mathcal{M}^{H}, \otimes, \Psi^{R}\right)$ of $H$-comodules. That is, $\varepsilon_{L}: L \rightarrow \mathbb{K}$ is an algebra map, $\eta_{L}: \mathbb{K} \rightarrow L$ a coalgebra map and moreover $\Delta_{L}$ is an algebra map with respect to the product $m_{L}$ in $L$ and the product $m_{L \otimes L}=\left(m_{L} \otimes m_{L}\right) \circ\left(\mathrm{id}_{L} \otimes \Psi_{L, L}^{R} \otimes \mathrm{id}_{L}\right)$ in $L \otimes L($ as given in (2.15) $)$, that is

$$
\Delta_{L} \circ m_{L}=m_{L \otimes L} \circ\left(\Delta_{L} \otimes \Delta_{L}\right) .
$$

The braided biagebra $L$ is a braided Hopf algebra if there is a map $S_{L}: L \rightarrow L$, called antipode or braided antipode, that satisfies the antipode property (of being the convolution inverse of the identity id : $L \rightarrow L$ ):

$$
m_{L} \circ\left(\operatorname{id}_{L} \otimes S_{L}\right) \circ \Delta_{L}=\eta_{L} \circ \varepsilon_{L}=m_{L} \circ\left(S_{L} \otimes \operatorname{id}_{L}\right) \circ \Delta_{L},
$$

and that in addition is an $H$-comodule map.

For later use we recall that the antipode $S_{L}: L \rightarrow L$ of a braided Hopf algebra $L$ is a braided anti-algebra map and a braided anti-coalgebra map

$$
S_{L} \circ m_{L}=m_{L} \circ \Psi_{L, L}^{R} \circ\left(S_{L} \otimes S_{L}\right), \quad \Delta_{L} \circ S_{L}=\left(S_{L} \otimes S_{L}\right) \circ \Psi_{L, L}^{R} \circ \Delta_{L} .
$$

Example 2.14. The braided Hopf algebra $\underline{H}$ of a coquasitriangular Hopf algebra $(H, R)$. Recall that for any Hopf algebra $H$, the data $(H, \Delta, \varepsilon, \mathrm{Ad})$ is an $H$-comodule coalgebra and that for $H$ coquasitriangular $(\underline{H}, \dot{-}, \mathrm{Ad})$ is an $H$-comodule algebra. These two structures define the braided Hopf algebra

$$
(\underline{H}, \dot{-}, \eta, \Delta, \varepsilon, \underline{S}, \mathrm{Ad}) \text {. }
$$

Here, as $H$-comodule maps, both $\eta: \underline{H} \rightarrow \mathbb{K}$ and $\Delta: \underline{H} \rightarrow \underline{H} \otimes \underline{H}$ are the same as the counit and coproduct in $H$, with now $\Delta$ considered as an algebra map for the product . in $\underline{H}$ and the -product $m_{\underline{H} \otimes \underline{H}}$ in $\underline{H} \otimes \underline{H}$. The antipode $\underline{S}:=S_{\underline{H}}: \underline{H} \rightarrow \underline{H}$ can be shown to be given, for all $h \in H$, by

$$
\underline{S}(h):=S\left(h_{(2)}\right) R\left(S^{2}\left(h_{(3)}\right) S\left(h_{(1)}\right) \otimes h_{(4)}\right) .
$$

Lemma 2.15. The braided Hopf algebra $\underline{H}$ is braided commutative, that is, for all $h, k \in \underline{H}$, its product satisfies

$$
k_{(2)} \div h_{(2)} R\left(S\left(k_{(1)}\right) k_{(3)} \otimes h_{(1)}\right)=h_{(1)} \div k_{(2)} \bar{R}\left(h_{(2)} \otimes S\left(k_{(1)}\right) k_{(3)}\right),
$$

this equation being equivalent to (2.7). 
Proof. By substituting (2.18) in (2.7) and using the basic properties of the $R$-form $R$, one obtains:

$$
\begin{aligned}
k h & =R\left(h_{(1)} \otimes k_{(1)}\right) h_{(2)} k_{(2)} \bar{R}\left(h_{(3)} \otimes k_{(3)}\right) \\
\Longleftrightarrow & k_{(2)} \cdot h_{(2)} R\left(S\left(k_{(1)}\right) k_{(3)} \otimes h_{(1)}\right) \\
& =R\left(h_{(1)} \otimes k_{(1)}\right) h_{(3)} \cdot k_{(3)} R\left(S\left(h_{(2)}\right) h_{(4)} \otimes k_{(2)}\right) \bar{R}\left(h_{(5)} \otimes k_{(5)}\right) \\
\Longleftrightarrow & k_{(2)} \cdot h_{(2)} R\left(S\left(k_{(1)}\right) k_{(3)} \otimes h_{(1)}\right)=R\left(h_{(2)} \otimes k_{(1)}\right) h_{(1)} \cdot k_{(2)} \bar{R}\left(h_{(3)} \otimes k_{(3)}\right) \\
\Longleftrightarrow & k_{(2)} \cdot h_{(2)} R\left(S\left(k_{(1)}\right) k_{(3)} \otimes h_{(1)}\right)=h_{(1)} \div k_{(2)} \bar{R}\left(h_{(2)} \otimes S\left(k_{(1)}\right) k_{(3)}\right)
\end{aligned}
$$

where in the last passage we used the analogous properties in (2.8) for $\bar{R}$. Thus $\underline{H}$ is braided commutative.

Lemma 2.16. The following conditions are equivalent to (2.24)

$$
\begin{aligned}
& h \cdot k=k_{(3)} \cdot h_{(2)} R\left(S\left(k_{(2)}\right) k_{(4)} \otimes h_{(1)}\right) R\left(h_{(3)} \otimes S\left(k_{(1)}\right) k_{(5)}\right), \\
& h \cdot k=k_{(3)} \div h_{(3)} R\left(S\left(h_{(2)}\right) h_{(4)} \otimes S\left(k_{(1)}\right) k_{(5)}\right) Q\left(h_{(1)} \otimes S\left(k_{(2)}\right) k_{(4)}\right),
\end{aligned}
$$

where $Q$ is the convolution product $Q=R_{21} * R$.

Proof. The implication $(2.25) \Rightarrow(2.24)$ is proven by substituting in the right hand side of (2.24) the expression for $h_{(1)} \div k_{(2)}$ given by relation (2.25).

For the converse implication $(2.24) \Rightarrow(2.25)$ we compute

$$
\begin{aligned}
h \cdot k & =h_{(1)} \cdot k_{(2)} \varepsilon\left(h_{(2)}\right) \varepsilon\left(S\left(k_{(1)}\right) k_{(3)}\right) \\
& =h_{(1)} \cdot k_{(3)} \bar{R}\left(h_{(2)} \otimes S\left(k_{(2)}\right) k_{(4)}\right) R\left(h_{(3)} \otimes S\left(k_{(1)}\right) k_{(5)}\right) \\
& =k_{(3)} \cdot h_{(2)} R\left(S\left(k_{(2)}\right) k_{(4)} \otimes h_{(1)}\right) R\left(h_{(3)} \otimes S\left(k_{(1)}\right) k_{(5)}\right),
\end{aligned}
$$

where in the last equality we used (2.24).

Equivalence of (2.26) and (2.25) is shown by using the explicit convolution product $Q$ :

$$
\begin{aligned}
h . k & =k_{(3)} \cdot h_{(3)} R\left(S\left(h_{(2)}\right) h_{(4)} \otimes S\left(k_{(1)}\right) k_{(5)}\right) Q\left(h_{(1)} \otimes S\left(k_{(2)}\right) k_{(4)}\right) \\
& =k_{(4)} \cdot h_{(4)} R\left(S\left(h_{(3)}\right) h_{(5)} \otimes S\left(k_{(1)}\right) k_{(7)}\right) R\left(S\left(k_{(3)}\right) k_{(5)} \otimes h_{(1)}\right) R\left(h_{(2)} \otimes S\left(k_{(2)}\right) k_{(6)}\right) \\
& =k_{(3)} \cdot h_{(4)} R\left(h_{(2)} S\left(h_{(3)}\right) h_{(5)} \otimes S\left(k_{(1)}\right) k_{(5)}\right) R\left(S\left(k_{(2)}\right) k_{(4)} \otimes h_{(1)}\right) \\
& =k_{(3)} \cdot h_{(2)} R\left(S\left(k_{(2)}\right) k_{(4)} \otimes h_{(1)}\right) R\left(h_{(3)} \otimes S\left(k_{(1)}\right) k_{(5)}\right),
\end{aligned}
$$

where we used the basic property (2.8) of the $R$-form in the third equality.

We next introduce the notion of quasi-commutative algebra $A \in \mathcal{A}^{H}$ and provide a few examples.

Definition 2.17. Let $(H, R)$ be a coquasitriangular Hopf algebra. A right $H$-comodule algebra $A \in \mathcal{A}^{H}$ is quasi-commutative (for the coquasitriangular structure $R$ of $H$ ) if

$$
m_{A}=m_{A} \circ\left(\Psi_{A, A}^{R}\right)^{-1}, \quad a c=c_{(0)} a_{(0)} \bar{R}\left(c_{(1)} \otimes a_{(1)}\right)
$$

or equivalently

$$
m_{A}=m_{A} \circ \Psi_{A, A}^{R}, \quad a c=c_{(0)} a_{(0)} R\left(a_{(1)} \otimes c_{(1)}\right)
$$

for all $a, c \in A$. We denote by $\mathcal{A}_{q c}^{(H, R)}$ the full subcategory of $\mathcal{A}^{H}$ of quasi-commutative comodule algebras (for the coquasitriangular structure $R$ ), where morphisms are $H$ comodule algebra morphisms. 
The first expression (2.27) implies the second (2.28):

$$
a c=a_{(0)} c_{(0)} \varepsilon\left(a_{(1)} c_{(1)}\right)=a_{(0)} c_{(0)} \bar{R}\left(a_{(1)} \otimes c_{(1)}\right) R\left(a_{(2)} \otimes c_{(2)}\right)=c_{(0)} a_{(0)} R\left(a_{(1)} \otimes c_{(1)}\right) .
$$

Similarly the second expression implies the first one. For future use (see Theorem 2.29) we also prove a third equivalent expression:

$$
c_{(0)} a_{(0)} \otimes c_{(2)} R\left(S\left(c_{(1)}\right) c_{(3)} \otimes a_{(1)}\right)=a_{(0)} c_{(0)} \otimes c_{(1)} R\left(c_{(2)} \otimes a_{(1)}\right) .
$$

Indeed, (2.27) implies (2.29):

$$
\begin{aligned}
a_{(0)} c_{(0)} \otimes c_{(1)} R\left(c_{(2)} \otimes a_{(1)}\right) & =c_{(0)} a_{(0)} \bar{R}\left(c_{(1)} \otimes a_{(1)}\right) \otimes c_{(2)} R\left(c_{(3)} \otimes a_{(2)}\right) \\
& =c_{(0)} a_{(0)} R\left(S\left(c_{(1)}\right) \otimes a_{(1)}\right) \otimes c_{(2)} R\left(c_{(3)} \otimes a_{(2)}\right) \\
& =c_{(0)} a_{(0)} \otimes c_{(2)} R\left(S\left(c_{(1)}\right) c_{(3)} \otimes a_{(1)}\right) .
\end{aligned}
$$

On the other hand, id $\otimes \varepsilon$ applied to (2.29) and the normalization $R(1 \otimes h)=\varepsilon(h)$ give (2.28).

The quasi-commutativity property of $A \in \mathcal{A}^{H}$ can be equivalently characterized as the compatibility of the multiplication in $A$ with that in the braided tensor product $A \otimes A$ :

Proposition 2.18. Let $(H, R)$ be a coquasitriangular Hopf algebra. An H-comodule algebra $\left(A, m_{A}, \delta^{A}\right)$ is quasi-commutative if and only if the multiplication $m_{A}: A \otimes A \rightarrow A$, $a \bigotimes c \mapsto a c$ is an algebra map. Thus $m_{A}: A \otimes A \rightarrow A$ is an H-comodule algebra map if $A \in \mathcal{A}_{q c}^{(H, R)}$.

Proof. On the one hand

$$
m_{A}\left((a \otimes c) \cdot\left(a^{\prime} \otimes c^{\prime}\right)\right)=m_{A}\left(a a_{(0)}^{\prime} \otimes c_{(0)} c^{\prime} R\left(c_{(1)} \otimes a_{(1)}^{\prime}\right)\right)=a a_{(0)}^{\prime} c_{(0)} c^{\prime} R\left(c_{(1)} \otimes a_{(1)}^{\prime}\right) ;
$$

on the other hand

$$
\left(m_{A}(a \otimes c)\right)\left(m_{A}\left(a^{\prime} \otimes c^{\prime}\right)\right)=a c a^{\prime} c^{\prime} .
$$

Hence the two expressions coincide if and only if $A$ is quasi-commutative. Moreover, by definition of $H$-comodule algebra, the multiplication map is an $H$-comodule map.

Remark 2.19. The subalgebra $A^{c o H} \subseteq A$ of a quasi-commutative $H$-comodule algebra $A$ is contained in the centre $Z(A)$ of $A$. This follows from (2.27) and the normalization property (2.10) of the $R$-form.

Example 2.20. Every commutative algebra $A \in \mathcal{A}^{H}$, with commutative Hopf algebra $H$ and trivial coquasitriangular structure $R=\varepsilon \otimes \varepsilon$, is quasi-commutative. Indeed quasicommutativity with $R=\varepsilon \otimes \varepsilon$ is equivalent to commutativity.

Example 2.21. Let $(H, R)$ be a coquasitriangular Hopf algebra, the $H$-comodule algebra $(H, \cdot, \Delta)$ is quasi-commutative if and only if $R=\varepsilon \otimes \varepsilon$ is the trivial $R$-form, and hence $H$ is commutative. The proof is straighforward, comparing the cotriangularity and quasi-commutativity conditions (2.7) and (2.27) we obtain, for all $h, k \in H$, $h k=R\left(h_{(1)} \otimes k_{(1)}\right) h_{(2)} k_{(2)}$. Applying the counit $\varepsilon$ gives $R=\varepsilon \otimes \varepsilon$, and hence commutativity of $H$.

Many examples of quasi-commutative algebras arise as twist deformations (see 84.1 ) of commutative algebras $A \in \mathcal{A}^{H}$. More in general, twist deformations of quasi-commutative algebras are quasi-commutative algebras:

Example 2.22. Let $A \in \mathcal{A}_{q c}^{(H, R)}$ and $\gamma: H \otimes H \rightarrow \mathbb{K}$ a 2-cocycle on $H$. Consider the Hopf algebra $H_{\gamma}$ with coquasitriangular structure $R_{\gamma}=\gamma_{21} * R * \bar{\gamma}$ as in Example 2.7. Let $A_{\gamma} \in \mathcal{A}^{H_{\gamma}}$ be the twisted $H_{\gamma}$-comodule algebra of $A$ : this is the $\mathbb{K}$-module $A$ with new 
product $a \bullet \gamma a^{\prime}:=a_{(0)} a_{(0)}^{\prime} \bar{\gamma}\left(a_{(1)} \otimes a_{(1)}^{\prime}\right)$ and unchanged coaction $a \mapsto a_{(0)} \otimes a_{(1)}$ (see §4.1). Then,

$$
\begin{aligned}
a \bullet_{\gamma} a^{\prime} & =a_{(0)}^{\prime} a_{(0)} R\left(a_{(1)} \otimes a_{(1)}^{\prime}\right) \bar{\gamma}\left(a_{(2)} \otimes a_{(2)}^{\prime}\right) \\
& =a_{(0)}^{\prime} \bullet_{\gamma} a_{(0)} \gamma\left(a_{(1)}^{\prime} \otimes a_{(1)}\right) R\left(a_{(2)} \otimes a_{(2)}^{\prime}\right) \bar{\gamma}\left(a_{(3)} \otimes a_{(3)}^{\prime}\right) \\
& =a_{(0)}^{\prime} \bullet_{\gamma} a_{(0)} R_{\gamma}\left(a_{(1)} \otimes a_{(1)}^{\prime}\right)
\end{aligned}
$$

showing that $A_{\gamma} \in \mathcal{A}_{q c}^{\left(H_{\gamma}, R_{\gamma}\right)}$.

Example 2.23. Let $H$ be commutative with trivial $R$-form $R=\varepsilon \otimes \varepsilon$, so that the $H$ comodule algebra $(H, \cdot, \Delta)$ is quasi-commutative (cf. Example 2.20). The twist deformation of $(H, \cdot, \Delta) \in \mathcal{A}_{q c}^{(H, \varepsilon \otimes \varepsilon)}$, as in Example 2.22 just above, gives the quasi-commutative $H_{\gamma}$-comodule algebra $\left(H, \bullet_{\gamma}, \Delta\right) \in \mathcal{A}_{q c}^{\left(H_{\gamma}, R_{\gamma}\right)}$, with $R_{\gamma}=\gamma_{21} * \bar{\gamma}$.

Example 2.24. A main example of quasi-commutative comodule algebra is the $H$-comodule algebra $(\underline{H}, \dot{-}, \mathrm{Ad})$ associated with a cotriangular Hopf algebra $(H, R)$. Indeed cotriangularity reads $Q=\varepsilon \otimes \varepsilon$ and then the braided commutativity property (2.26) reduces to the quasi-commutativity property

$$
h \cdot k=k_{(2)} \div h_{(2)} R\left(S\left(h_{(1)}\right) h_{(3)} \otimes S\left(k_{(1)}\right) k_{(3)}\right) .
$$

Quasi-commutativity of $\underline{H}$ does not imply cotriangularity of $H$ as this example shows:

Example 2.25. Let $H=\mathbb{C} \mathbb{Z}=\mathbb{C}\left[g, g^{-1}\right]$ be the group Hopf-algebra of the group $\mathbb{Z}$ considered in Example 2.5, with $R$-form $R_{q}\left(g^{n}, g^{m}\right)=q^{-n m}$ for a complex number $q \neq 1$. It is coquasitriangular but not cotriangular. Since the adjoint coaction is trivial it is immediate that $(\underline{H}=\mathbb{C} \mathbb{Z}, \cdot, \mathrm{Ad})$ is quasi-commutative with respect to $R_{q}$. More generally, if $R$ is a coquasitriangular structure on a commutative and cocommutative algebra $H$, then $(\underline{H}, \dot{-}, \mathrm{Ad})=(H, \cdot, \mathrm{Ad})$ is quasi-commutative since the adjoint coaction is trivial.

Another example of quasi-commutative algebra $A \in \mathcal{A}^{H}$ with coquasitriangular and not cotriangular Hopf algebra $H$ is the following one:

Example 2.26. The FRT bialgebra $\mathcal{O}\left(M_{q}(2)\right)$ is generated for $j, k=1,2$, by elements $u_{j k}$, satisfying $\mathcal{R}_{k l}^{j i} u_{k m} u_{l n}=u_{i k} u_{j l} \mathcal{R}_{m n}^{l k}$, with the only non zero components of the matrix $\mathcal{R}$

$$
\mathcal{R}_{11}^{11}=\mathcal{R}_{22}^{22}=q \quad, \quad \mathcal{R}_{12}^{12}=\mathcal{R}_{21}^{21}=1 \quad, \quad \mathcal{R}_{12}^{21}=q-q^{-1}
$$

for $q \in \mathbb{C}, q \neq 0$. Let $H=\mathcal{O}\left(G L_{q}(2)\right)$ be the Hopf algebra of coordinate functions of the quantum group $G L_{q}(2)$ which is obtained by extending $\mathcal{O}\left(M_{q}(2)\right)$ by a central element $D^{-1}$, defined to be the inverse of the quantum determinant $D:=u_{11} u_{22}-q u_{12} u_{21}$. The Hopf algebra $H$ is coquasitriangular with (not cotriangular) universal $R$-form

$$
R\left(u_{i j} \otimes u_{k l}\right)=q^{-1} \mathcal{R}_{j l}^{i k}, \quad R\left(D^{-1} \otimes u_{i j}\right)=R\left(u_{i j} \otimes D^{-1}\right)=q \delta_{i j},
$$

see e.g. [20, §10.1]. The convolution inverse is $\bar{R}\left(u_{i j} \otimes u_{k l}\right)=q\left(\mathcal{R}^{-1}\right)_{j l}^{i k}$. Let $A=\mathcal{O}\left(\mathbb{C}_{q}^{2}\right)$ be the algebra of the quantum plane, that is, the algebra generated by two elements $x_{1}, x_{2}$ with commutation relations $x_{1} x_{2}=q x_{2} x_{1}$. It is well known that $A$ is a $\mathcal{O}\left(G L_{q}(2)\right)$ comodule algebra with coaction $\delta\left(x_{i}\right)=x_{j} \otimes u_{j i}$; it is easily verified that $A$ is quasicommutative with respect to the coquasitriangular structure $R$ defined in (2.30):

$$
x_{i} x_{j}=x_{l} x_{p} R\left(u_{p i} \otimes u_{l j}\right)=q^{-1} \mathcal{R}_{i j}^{p l} x_{l} x_{p}
$$

for each $i, j=1,2$. Note that the Hopf algebra $\mathcal{O}\left(G L_{q}(2)\right)$ admits the one parameter family of coquasitriangular structures $R_{\lambda}\left(u_{i j} \otimes u_{k l}\right)=\lambda \mathcal{R}_{j l}^{i k}$, with nonvanishing $\lambda \in \mathbb{C}$. 
For $\lambda$ a square root of $q^{-1}, R_{\lambda}$ is also a coquasitriangular structure on the quotient Hopf algebra $\mathcal{O}\left(S L_{q}(2)\right)$. Nevertheless, the comodule algebra $A=\mathcal{O}\left(\mathbb{C}_{q}^{2}\right)$ is not quasicommutative with respect to it.

\subsection{Hopf-Galois extensions for coquasitriangular Hopf algebras.}

As mentioned in $\$ 2.1$, for a generic noncommutative algebra extension, in contrast with the commutative case, the canonical map $\chi=\left(m_{A} \otimes \mathrm{id}\right) \circ(\mathrm{id} \otimes \delta)$ is just a morphism of relative Hopf modules. The domain $A \otimes_{B} A$ of $\chi$ itself does not inherit an algebra structure from $A \otimes A$ and the multiplication $m_{A}: A \otimes_{B} A \rightarrow A$ is not an algebra map. In this subsection we find when the canonical map of an Hopf-Galois extension with coquasitriangular Hopf algebra is an algebra map, and a morphism in the category $\left(\mathcal{A}^{H}, \bigotimes\right)$.

Lemma 2.27. Let $(H, R)$ be a coquasitriangular Hopf algebra, and let $A \in \mathcal{A}^{H}$ with subalgebra of coinvariants $B=A^{c o H} \subseteq A$. The - product (2.15) on $A \otimes A$ descends to a well-defined product on $A \otimes_{B} A$ if and only if $B$ is in the centre of $A$.

Proof. The balanced tensor product $A \otimes_{B} A$ is by definition the quotient of $A \otimes A \in{ }_{A} \mathcal{M}_{A}{ }^{H}$ by the $A$-sub-bimodule and $H$-subcomodule $J=\left\{a(b \otimes 1-1 \otimes b) a^{\prime}, a, a^{\prime} \in A, b \in B\right\}$. We prove the lemma by showing that $J$ is an ideal in $A \otimes A$ if and only if the subalgebra of coinvariants $B$ is central in $A$. If $J$ is an ideal in $A \otimes A$ then, for all $a \in A, b \in B$, $(b \otimes 1-1 \otimes b) \cdot(a \otimes 1) \in J ;$ since $B=A^{c o H}$, and thus $(b \otimes 1-1 \otimes b) \cdot(a \otimes 1)=$ $-[a, b] \otimes 1+a(b \otimes 1-1 \otimes b)$, this implies $[a, b] \otimes 1=0$ and hence $[a, b]=0$. Vice versa if $B$ is central in $A$ then for all $a, a^{\prime} \in A, b \in B$

$$
a(b \otimes 1-1 \otimes b) a^{\prime}=\left(a \otimes a^{\prime}\right)(b \otimes 1-1 \otimes b)=\left(a \otimes a^{\prime}\right) \cdot(b \otimes 1-1 \otimes b)
$$

where the last equality holds because $B=A^{c o H}$. In a similar way, $a(b \otimes 1-1 \otimes b) a^{\prime}=$ $(b \otimes 1-1 \otimes b) \cdot\left(a \otimes a^{\prime}\right)$. This proves that $J$ is the two-sided ideal in $A \otimes A$ generated by $b \otimes 1-1 \otimes b, b \in B$.

Since Remark 2.19 shows that the subalgebra of coinvariants of a quasi-commutative algebra $A \in \mathcal{A}_{q c}^{(H, R)}$ is in the centre of $A$, for such an algebra $A$ we have that $A \otimes_{B} A$ inherits an algebra structure from $A \otimes A$; we denote it by $A \bigotimes_{B} A$. We correspondingly denote by $\delta^{A \otimes_{B} A}: A \bigotimes_{B} A \rightarrow A \bigotimes_{B} A \otimes H$ the $H$-coaction $\delta^{A \otimes_{B} A}: A \otimes_{B} A \rightarrow A \otimes_{B} A \otimes H$.

Proposition 2.28. Let $(H, R)$ be a coquasitriangular Hopf algebra, and let $A \in \mathcal{A}_{q c}^{(H, R)}$ with subalgebra of coinvariants $B=A^{\mathrm{coH}}$. Then $\left(A \bigotimes_{B} A, \mathbf{\bullet}, \delta^{A \bigotimes_{B} A}\right)$ is an $H$-comodule algebra.

Proof. The triple $\left(A \otimes A, \mathbf{-}, \delta^{A \otimes A}\right)$ is an $H$-comodule algebra because $\left(\mathcal{A}^{H}, \bigotimes\right)$ is a monoidal category for $H$ coquasitriangular (cf. Proposition 2.9). The balanced tensor product $A \bigotimes_{B} A$ is the quotient of $A \otimes A$ via the ideal and $H$-subcomodule $J$ generated by $b \otimes 1-1 \otimes b, b \in B$. The $H$-comodule algebra structure on the quotient $A \bigotimes_{B} A$ is therefore induced from that of $A \otimes A$.

The results on $H$-comodule algebras and morphisms established so far are profitably applied to the study of the canonical map. Recalling the map $\underline{\delta}^{A}: A \rightarrow A \otimes \underline{H}$ associated with an $H$-comodule algebra $A$ (cf. Proposition 2.12), we have:

Theorem 2.29. Let $(H, R)$ be a coquasitriangular Hopf algebra and $A \in \mathcal{A}_{q c}^{(H, R)}$ a quasicommutative $H$-comodule algebra. Let $B=A^{\text {coH }}$ be the corresponding subalgebra of coinvariants. Then the canonical map

$$
\chi=(m \otimes \mathrm{id}) \circ\left(\operatorname{id} \otimes_{B} \underline{\delta}^{A}\right): A \otimes_{B} A \longrightarrow A \bowtie \underline{H}, \quad a^{\prime} \otimes_{B} a \longmapsto a^{\prime} a_{(0)} \otimes a_{(1)}
$$


is a composition of (well-defined) $H$-comodule algebra maps and thus a morphism in $\mathcal{A}^{H}$.

Proof. The map (id $\left.\otimes \underline{\delta}^{A}\right): A \otimes A \rightarrow A \otimes A \otimes \underline{H}$ is an $H$-comodule algebra map because tensor product of $H$-comodule algebra maps (cf. Proposition 2.12 and Proposition 2.8 or 2.9). The quotient $A \bigotimes_{B} A$ is well defined because $B$ is central in $A \in \mathcal{A}_{q c}^{(H, R)}$ (cf. Remark 2.19). The induced map on the quotient (id $\left.\bigotimes_{B} \underline{\delta}^{A}\right): A \bigotimes_{B} A \rightarrow A \bigotimes_{B} A \otimes \underline{H}$ is well-defined because of $B$-linearity of $\underline{\delta}^{A}$.

From Proposition 2.18 we know that $m_{A}: A \otimes A \rightarrow A$ is an $H$-comodule algebra map when $A$ is quasi-commutative. It induces a well-defined $H$-comodule algebra map on the quotient $m: A \otimes_{B} A \rightarrow A$. Then $m \otimes$ id $: A \otimes_{B} A \otimes \underline{H} \rightarrow A \otimes \underline{H}$ is an $H$-comodule algebra map because tensor product of comodule algebra maps.

As a corollary of the above proposition, when $\chi$ is invertible, the translation map

$$
\tau=\left.\chi^{-1}\right|_{1 \otimes_{\underline{H}}}: \underline{H} \longrightarrow A \bigotimes_{B} A
$$

is an algebra map as well, $\tau(h \cdot k)=\tau(h) \cdot \tau(k)$, and hence an $H$-comodule algebra map.

Let us record some additional properties of the translation map. Being $\tau=\left.\chi^{-1}\right|_{1 \otimes H}$, one has $h^{<1>} h^{<2>}=\varepsilon(h) 1_{A}$, for any $h \in \underline{H}$. In addition, by combining properties (2.6) and (2.5) one also has

$$
h_{(1)}^{<1>} \otimes h_{(0)}^{<1>} \otimes_{B} h_{(0)}^{<2>} \otimes h_{(1)}^{<2>}=S\left(h_{(1)}\right) \otimes h_{(2)}^{<1>} \otimes_{B} h_{(2)}^{<2>} \otimes h_{(3)},
$$

for any $h \in \underline{H}$.

For the particular case of an Hopf-Galois extension $A^{c o H} \subseteq A$ with $H$ coquasitriangular and $A \in \mathcal{A}_{q c}^{(H, R)}$, using the property (2.31) and $h^{<1>} h^{<2>}=\varepsilon(h) 1_{A}$, the quasicommutativity of $A$ leads to

$$
h^{<2>} h^{<1>}=\bar{R}\left(S\left(h_{(1)}\right) \otimes h_{(2)}\right) 1_{A}=R\left(h_{(2)} \otimes S\left(h_{(1)}\right)\right) 1_{A} .
$$

For later use in Proposition [3.6, we prove the following key additional properties of the translation map. The first one (2.33) just concerns $\tau$ as a linear map, the second one (2.34) uses that $\tau$ is an algebra map.

Lemma 2.30. Let $\left(A, \delta^{A}\right) \in \mathcal{A}_{q c}^{(H, R)}$ be a quasi-commutative $H$-comodule algebra, with the extension $A^{\mathrm{coH}} \subseteq A$ a Hopf-Galois one. The translation map satisfies the identity

$$
\tau \circ \underline{S}=\Psi_{A, A}^{R} \circ \tau
$$

Moreover,

$$
\text { - } \circ\left(\left(\Psi_{A, A}^{R} \circ \tau\right) \otimes \tau\right) \circ \Delta_{\underline{H}}=\eta_{A \unrhd_{B} A} \circ \varepsilon_{\underline{H}}
$$

that is, for each $h \in \underline{H}$

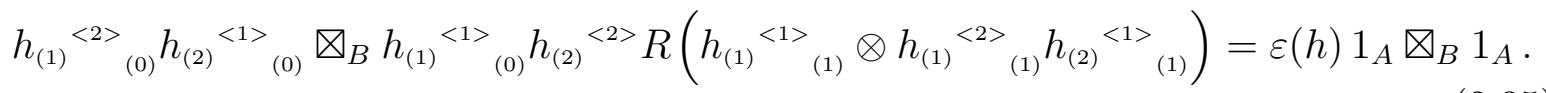


Proof. Identity (2.33) holds, indeed for each $h \in \underline{H}$ one has

$$
\begin{aligned}
\chi\left(\Psi_{A, A}^{R} \circ \tau(h)\right) & =\chi\left(h^{<2>}{ }_{(0)} \otimes_{B} h^{<1>}{ }_{(0)}\right) R\left(h^{<1>}{ }_{(1)} \otimes h^{<2>}{ }_{(1)}\right) \\
& =h^{<2>}{ }_{(0)}^{<1>} h_{(0)}^{<1>} h^{<1{ }_{(1)}} R\left(h^{<1>}{ }_{(2)} \otimes h^{<2>}{ }_{(1)}\right) \\
& =h_{(3)}{ }^{<2>} h_{(3)}{ }^{<1>} \otimes S\left(h_{(2)}\right) R\left(S\left(h_{(1)}\right) \otimes h_{(4)}\right) \\
& =1_{A} \otimes S\left(h_{(2)}\right) \bar{R}\left(S\left(_{(3)}\right) \otimes h_{(4)}\right) R\left(S\left(h_{(1)}\right) \otimes h_{(5)}\right) \\
& =1_{A} \otimes S\left(h_{(2)}\right) R\left(S^{2}\left(h_{(3)}\right) \otimes h_{(4)}\right) R\left(S\left(h_{(1)}\right) \otimes h_{(5)}\right) \\
& =1_{A} \otimes S\left(h_{(2)}\right) R\left(S^{2}\left(h_{(3)}\right) S\left(h_{(1)}\right) \otimes h_{(4)}\right) \\
& =1_{A} \otimes \underline{S}(h)
\end{aligned}
$$

where for the third equality we used (2.31) and for the fourth one property (2.32) of the translation map. Then, the identity (2.33) directly implies the second part of the lemma; indeed

$$
\begin{aligned}
\text { - } \circ\left(\left(\Psi_{A, A}^{R} \circ \tau\right) \otimes \tau\right) \circ \Delta_{\underline{H}} & =\bullet \circ((\tau \circ \underline{S}) \otimes \tau) \circ \Delta_{\underline{H}}=\cdot \circ(\tau \otimes \tau) \circ(\underline{S} \otimes \mathrm{id}) \circ \Delta_{\underline{H}} \\
& =\tau \circ=\circ(\underline{S} \otimes \mathrm{id}) \circ \Delta_{\underline{H}}=\tau \circ \eta_{\underline{H}} \circ \varepsilon_{\underline{H}}=\eta_{A \bigotimes_{B} A} \circ \varepsilon_{\underline{H}},
\end{aligned}
$$

using in the second line that $\tau$ is an algebra map and the antipode property (2.21).

Remark 2.31. If $H$ is commutative with trivial $R$-form, $R=\varepsilon \otimes \varepsilon$, then $A \in \mathcal{A}_{q c}^{(H, R)}$ is commutative and property (2.33) simply reads $\tau(S(h))=$ flip $\circ \tau(h) \in A \otimes_{B} A$. In particular, let $B=A^{c o H} \subseteq A$ be the Hopf-Galois extension of the principal $G$-bundle $\pi: P \rightarrow P / G$ considered in Example 2.2. Then the property $\tau(S(h))=$ flip $\circ \tau(h)$ of the pull-back $\tau=t^{*}$ of the classical translation map corresponds by duality to the property $t(q, p)=t(p, q)^{-1}, p, q \in P$. Similarly, property (2.34) corresponds by duality to $t(q, p) t(p, q)=e$, the neutral element of $G$.

When $(H, R)$ is a cotriangular bialgebra, the category $\mathcal{A}_{q c}^{(H, R)}$ of quasi-commutative $H$-comodule algebras with the braided tensor product $\otimes$ becomes a braided monoidal category. Moreover, the canonical map is a morphism in $\mathcal{A}_{q c}^{(H, R)}$.

Proposition 2.32. Let $(H, R)$ be a cotriangular bialgebra. The braided tensor product of quasi-commutative $H$-comodule algebras is a quasi-commutative $H$-comodule algebra.

Proof. Let $A, C \in \mathcal{A}^{H}$ be quasi-commutative, then for all $a, a^{\prime} \in A$ and $c, c^{\prime} \in C$,

$$
\begin{aligned}
(a \otimes c) \cdot\left(a^{\prime} \otimes c^{\prime}\right) & =a a_{(0)}^{\prime} \otimes c_{(0)} c^{\prime} R\left(c_{(1)} \otimes a_{(1)}^{\prime}\right) \\
& =a_{(0)}^{\prime} a_{(0)} \otimes c^{\prime}{ }_{(0)} c_{(0)} R\left(a_{(1)} \otimes a_{(1)}^{\prime}\right) R\left(c_{(1)} \otimes c_{(1)}^{\prime}\right) R\left(c_{(2)} \otimes a_{(2)}^{\prime}\right)
\end{aligned}
$$

where we used the definition of the -product in (2.15) and the quasi commutativity of $A$ and $C$. On the other hand,

$$
\begin{aligned}
& \left(a^{\prime} \otimes c^{\prime}\right)_{(0)} \cdot(a \otimes c)_{(0)} R\left(\left(a \otimes c_{(1)}\right) \otimes\left(a^{\prime} \otimes c^{\prime}\right)_{(1)}\right)= \\
& =\left(a_{(0)}^{\prime} \otimes c^{\prime}{ }_{(0)}\right) \cdot{ }^{\prime}\left(a_{(0)} \otimes c_{(0)}\right) R\left(a_{(1)} c_{(1)} \otimes a_{(1)}^{\prime} c_{(1)}^{\prime}\right) \\
& =a_{(0)}^{\prime} a_{(0)} \otimes c^{\prime}{ }_{(0)} c_{(0)} R\left(c^{\prime}{ }_{(1)} \otimes a_{(1)}\right) R\left(a_{(3)} \otimes a_{(1)}^{\prime}\right) R\left(a_{(2)} \otimes c_{(2)}^{\prime}\right) R\left(c_{(1)} \otimes c_{(3)}^{\prime}\right) R\left(c_{(2)} \otimes a_{(2)}^{\prime}\right) .
\end{aligned}
$$

This coincides with the previous expression since, using the cotriangularity of $H$, one can simplify $R\left(c_{(1)}^{\prime} \otimes a_{(1)}\right) R\left(a_{(2)} \otimes c_{(2)}^{\prime}\right)=\bar{R}\left(a_{(1)} \otimes c_{(1)}^{\prime}\right) R\left(a_{(2)} \otimes c_{(2)}^{\prime}\right)=\varepsilon\left(a_{(1)}\right) \varepsilon\left(c_{(1)}^{\prime}\right)$.

As a direct consequence of this proposition we have:

Corollary 2.33. Let $(H, R)$ be a cotriangular Hopf algebra. The category $\mathcal{A}_{q c}^{(H, R)}$ endowed with the braided tensor product $\otimes$ is a full sub-monoidal category of $\left(\mathcal{A}^{H}, \otimes\right)$. 
From Theorem 2.29 we then have:

Corollary 2.34. Let $(H, R)$ be a cotriangular Hopf algebra, $A \in \mathcal{A}_{q c}^{(H, R)}$ and $B=A^{c o H} \subseteq$ A a Hopf-Galois extension. Then $\underline{H} \in \mathcal{A}_{q c}^{(H, R)}$ and the corresponding canonical map $\chi: A \bigotimes_{B} A \longrightarrow A \otimes \underline{H}$ is an isomorphism in the category $\left(\mathcal{A}_{q c}^{(H, R)}, \bigotimes\right)$.

\section{The gauge Group}

In the classical (commutative) case one way to define the group $\mathcal{G}_{P}$ of gauge transformations of a principal $G$-bundle $\pi: P \rightarrow P / G$ is as the group of $G$-equivariant maps,

$$
\mathcal{G}_{P}:=\left\{\sigma: P \rightarrow G ; \sigma(p g)=g^{-1} \sigma(p) g\right\},
$$

where $G$ is a right $G$-space with respect to the right $G$-adjoint action. The group structure is by point-wise product: $(\sigma \tilde{\sigma})(p)=\sigma(p) \tilde{\sigma}(p)$, for all $\sigma, \tilde{\sigma} \in \mathcal{G}_{P}$ and $p \in P$. The gauge group can be equivalently defined as the subgroup of principal bundle automorphisms which are vertical (project to the identity on the base space):

$$
\operatorname{Aut}_{P / G}(P):=\{\varphi: P \rightarrow P ; \varphi(p g)=\varphi(p) g, \pi(\varphi(p))=\pi(p)\},
$$

with group law given by map composition. The equivalence of these two definitions is well known [17, §7.1].

These definitions can be dualised for algebras rather than spaces. For instance, in the context of the affine varieties case treated in Example 2.2, where $A=\mathcal{O}(P), B=$ $\mathcal{O}(P / G), H=\mathcal{O}(G)$, the gauge group $\mathcal{G}_{P}$ in (3.1) of $G$-equivariant maps corresponds to that of $H$-equivariant maps (or $H$-comodule maps) that are also algebra maps

$$
\mathcal{G}_{A}:=\left\{\mathrm{f}: H \rightarrow A ; \delta^{A}(\mathrm{f})=(\mathrm{f} \otimes \mathrm{id}) \circ \mathrm{Ad}, \mathrm{f} \text { algebra map }\right\} .
$$

The group structure is the convolution product. The algebra map property for the pullback $\mathrm{f}=\sigma^{*}: H \rightarrow A$ comes from the point-wise product in $H=\mathcal{O}(G)$ and $A=\mathcal{O}(P)$ : $\mathbf{f}(h k)(p)=(h k)(\sigma(p))=h(\sigma(p) k(\sigma(p))=(\mathbf{f}(h) \mathbf{f}(k))(p)$, for all $h, k \in H, p \in P$.

Similarly, the vertical automorphisms description (3.2) of the gauge group corresponds to that of $H$-equivariant maps

$$
\operatorname{Aut}_{B}(A)=\left\{\mathrm{F}: A \rightarrow A ; \delta^{A} \mathrm{~F}=(\mathrm{F} \otimes \mathrm{id}) \delta^{A},\left.\mathrm{~F}\right|_{B}=\mathrm{id}: B \rightarrow B, \mathrm{~F} \text { algebra map }\right\} .
$$

The dual definitions can be given for a general Hopf-Galois extension $B=A^{c o H} \subseteq$ $A$, with $A$ and $H$ commutative algebras. However, for a noncommutative Hopf-Galois extension the algebra map condition in these definitions is in general very restrictive. This does not come as a surprise: for noncommutative algebras already algebra automorphisms are very constrained with respect to the commutative case.

In [3, §5] this issue was faced by weakening the notion of gauge group: gauge transformations are no longer algebra maps; they are defined to be comodule maps that are invertible and unital. In this "no algebra maps" context the isomorphism $\mathcal{G}_{A} \simeq \operatorname{Aut}_{B}(A)$ still holds. A drawback of this approach, besides the extra requirement of invertibility of the maps, is that the resulting gauge groups are very big, even in the classical case. For example the gauge group of the $G$-bundle on a point $G \rightarrow\{*\}$ is much bigger than the structure group $G$ as the following simple example shows.

Example 3.1. Consider the group $\mathbb{Z}_{2}:=\{e, u\}$ of integers modulo $2: e+e=u+u=$ $e, e+u=u+e=u$. Let $H$ be its coordinate Hopf algebra; this is the commutative complex algebra generated by the two orthogonal projections $p_{e}$ and $p_{u}$ (where $p_{a}(b)=\delta_{a, b}$, for $a, b \in \mathbb{Z}_{2}$ ) with unit $1_{H}=p_{e}+p_{u}$ the constant function 1 . It has cocommutative coproduct

$$
\Delta p_{e}=p_{e} \otimes p_{e}+p_{u} \otimes p_{u}, \quad \Delta p_{u}=p_{e} \otimes p_{u}+p_{u} \otimes p_{e}
$$


and counit $\varepsilon\left(p_{e}\right)=1, \varepsilon\left(p_{u}\right)=0$. The trivial $\mathbb{Z}_{2}$-bundle over a point is dually described as the Hopf-Galois extension $B \subseteq A$, where $A=H$ with coaction $\Delta$ and resulting algebra of coinvariants $B=\mathbb{C}$. Since $A=H$ is the linear span of $p_{e}$ and $p_{u}$ and the condition $\left.F\right|_{B}=$ id $: B \rightarrow B$ is just that of $\mathbb{C}$-linearity of the map $\mathrm{F}: A \rightarrow A$, we have linear maps $\mathrm{F}\left(x p_{e}+y p_{u}\right)=x^{\prime} p_{e}+y^{\prime} p_{u}$ from $\mathbb{C}^{2}$ to $\mathbb{C}^{2}$, that is, complex $2 \times 2$ matrices

$$
\left(\begin{array}{l}
x \\
y
\end{array}\right) \mapsto\left(\begin{array}{l}
x^{\prime} \\
y^{\prime}
\end{array}\right)=\left(\begin{array}{ll}
a & b \\
c & d
\end{array}\right)\left(\begin{array}{l}
x \\
y
\end{array}\right) \text {. }
$$

Unitality of $\mathrm{F}$ requires $b=1-a$ and $c=1-d$; invertibility of $\mathrm{F}$ requires $a+d \neq 1$. Finally, $H$ equivariance, that is $\Delta \mathrm{F}=(\mathrm{F} \otimes \mathrm{id}) \Delta$, leads to $a=d$. Summing up, the group of these maps is the $G L(2, \mathbb{C})$-subgroup

$$
\left\{\left(\begin{array}{cc}
a & 1-a \\
1-a & a
\end{array}\right), \quad \text { with } \quad 2 a \neq 1\right\} .
$$

If one imposes the additional condition that the maps $F$ are algebra maps this group collapses to a much smaller one. Indeed, the requirement

$$
\mathrm{F}\left(\left(x p_{e}+y p_{u}\right)\left(x^{\prime} p_{e}+y^{\prime} p_{u}\right)\right)=\mathrm{F}\left(x p_{e}+y p_{u}\right) \mathrm{F}\left(x^{\prime} p_{e}+y^{\prime} p_{u}\right)
$$

for all $(x, y)$ and $\left(x^{\prime}, y^{\prime}\right)$ in $\mathbb{C}^{2}$, forces $a=1$ or $a=0$. Thus the resulting group is

$$
\left\{\left(\begin{array}{ll}
1 & 0 \\
0 & 1
\end{array}\right),\left(\begin{array}{ll}
0 & 1 \\
1 & 0
\end{array}\right)\right\} \simeq \mathbb{Z}_{2}
$$

that is the expected group of gauge transformations.

We shall work in the noncommutative setting of the monoidal category $\left(\mathcal{A}^{H}, \otimes\right)$. In this context we show that it is natural to define the gauge group of vertical automorphisms as in (3.4), that is, to require vertical automorphisms $F$ to be algebra maps. Similarly, $\mathcal{G}_{A}$ is defined as the group of $H$-equivariant algebra maps $\mathrm{f}: \underline{H} \rightarrow A$. The issue of the lack of algebra maps is therefore in this case overcome by properly choosing the algebra structure on $H$, namely the multiplication - of the braided Hopf algebra $\underline{H}$ rather than that of the Hopf algebra $H$.

We begin by studying this latter space $\mathcal{G}_{A}$ of $H$-equivariant algebra maps. We then consider the gauge group $\operatorname{Aut}_{B}(A)$ of vertical automorphisms and prove its equivalence with $\mathcal{G}_{A}$. We present a few examples; while they are mainly commutative ones, they serve as a way of illustration of the notions involved. They will be deformed to noncommutative examples later on in the paper.

\subsection{The gauge group of equivariant algebra maps.}

Proposition 3.2. Let $(H, R)$ be a coquasitriangular Hopf algebra, $(\underline{H}, \dot{\sim}, \eta, \Delta, \varepsilon, \underline{S}, \mathrm{Ad})$ the associated braided Hopf algebra, $A \in \mathcal{A}_{q c}^{(H, R)}$ and $B=A^{c o H} \subseteq A$ a Hopf-Galois extension. The $\mathbb{K}$-module

$$
\mathcal{G}_{A}:=\operatorname{Hom}_{\mathcal{A}^{H}}(\underline{H}, A)
$$

of $H$-equivariant algebra maps $\underline{H} \rightarrow A$ is a group with respect to the convolution product. The inverse of $\mathrm{f} \in \mathcal{G}_{A}$ is given by $\overline{\mathrm{f}}:=\mathrm{f} \circ \underline{S}$.

Proof. Given $\mathrm{f}, \mathrm{g} \in \operatorname{Hom}_{\mathcal{A}^{H}}(\underline{H}, A)$, the product $\mathrm{f} * \mathrm{~g}$ is an $H$-comodule map; for all $h \in \underline{H}$,

$$
\begin{aligned}
\delta^{A}(\mathrm{f} * \mathrm{~g})(h) & =\delta^{A}\left(\mathrm{f}\left(h_{(1)}\right) \mathrm{g}\left(h_{(2)}\right)\right)=\delta^{A}\left(\mathrm{f}\left(h_{(1)}\right)\right) \delta^{A}\left(\mathrm{~g}\left(h_{(2)}\right)\right) \\
& =\left(\mathrm{f}\left(h_{(2)}\right) \otimes S\left(h_{(1)}\right) h_{(3)}\right)\left(\mathrm{g}\left(h_{(5)}\right) \otimes S\left(h_{(4)}\right) h_{(6)}\right)=\mathrm{f}\left(h_{(2)}\right) \mathrm{g}\left(h_{(3)}\right) \otimes S\left(h_{(1)}\right) h_{(4)}
\end{aligned}
$$


where we used that $\delta^{A}$ is an algebra morphism and that both $\mathrm{f}$ and $\mathrm{g}$ are $H$-comodule morphisms. Then

$$
\delta^{A}(\mathrm{f} * \mathrm{~g})(h)=(\mathrm{f} * \mathrm{~g})\left(h_{(2)}\right) \otimes S\left(h_{(1)}\right) h_{(3)}=\left((\mathrm{f} * \mathrm{~g}) \otimes \mathrm{id}_{H}\right) \operatorname{Ad}(h) .
$$

The product $\mathrm{f} * \mathrm{~g}$ is also an algebra map. Recall from (2.20) that $\Delta_{\underline{H}}$ is an algebra map with respect to the products - in $\underline{H}$ and - in the braided tensor product algebra $\underline{H} \otimes \underline{H}$, that is $\Delta_{\underline{H}}(h \cdot k)=h_{(1)} \cdot k_{(2)} \otimes h_{(3)} \cdot k_{(4)} R\left(S\left(h_{(2)}\right) h_{(4)} \otimes S\left(k_{(1)}\right) k_{(3)}\right)$ for all $h, k \in \underline{H}$. Then we compute

$$
\begin{aligned}
(\mathrm{f} * \mathrm{~g})(h \cdot k) & =\mathrm{f}\left(h_{(1)}-k_{(2)}\right) \mathrm{g}\left(h_{(3)} * k_{(4)}\right) R\left(S\left(h_{(2)}\right) h_{(4)} \otimes S\left(k_{(1)}\right) k_{(3)}\right) \\
& =\mathrm{f}\left(h_{(1)}\right) \mathrm{f}\left(k_{(2)}\right) \mathrm{g}\left(h_{(3)}\right) \mathrm{g}\left(k_{(4)}\right) R\left(S\left(h_{(2)}\right) h_{(4)} \otimes S\left(k_{(1)}\right) k_{(3)}\right) \\
& =\mathrm{f}\left(h_{(1)}\right)\left(\mathrm{f}\left(k_{(1)}\right)\right)_{(0)}\left(\mathrm{g}\left(h_{(2)}\right)\right)_{(0)} \mathrm{g}\left(k_{(2)}\right) R\left(\left(\mathrm{~g}\left(h_{(2)}\right)\right)_{(1)} \otimes\left(\mathrm{f}\left(k_{(1)}\right)\right)_{(1)}\right) \\
& =\mathrm{f}\left(h_{(1)}\right) \mathrm{g}\left(h_{(2)}\right) \mathrm{f}\left(k_{(1)}\right) \mathrm{g}\left(k_{(2)}\right) \\
& =(\mathrm{f} * \mathrm{~g})(h)(\mathrm{f} * \mathrm{~g})(k),
\end{aligned}
$$

where the second equality uses that $\mathrm{f}, \mathrm{g}$ are algebra maps, and the third equality that they are $H$-comodule maps. The fourth one follows from the quasi-commutativity of $A$.

Any $\mathrm{f}: \underline{H} \rightarrow A$ is convolution invertible, with inverse $\overline{\mathrm{f}}:=\mathrm{f} \circ \underline{S}$; indeed (recalling the antipode defining property (2.21) $)$

$$
(\mathbf{f} * \overline{\mathbf{f}})(h)=\mathbf{f}\left(h_{(1)}\right) \mathbf{f}\left(\underline{S}\left(h_{(2)}\right)\right)=\mathbf{f}\left(h_{(1)} \dot{-S}\left(h_{(2)}\right)\right)=\varepsilon(h) 1_{A},
$$

and similarly $\overline{\mathrm{f}} * \mathrm{f}=1_{A} \varepsilon$. The map $\overline{\mathrm{f}}$ is an $H$-comodule map being composition of $H$ comodule maps. In order to prove that $\overline{\mathrm{f}} \in \mathcal{G}_{\mathcal{A}}=\operatorname{Hom}_{\mathcal{A}^{H}}(\underline{H}, A)$ we are left to show that $\overline{\mathrm{f}}$ is an algebra map. This is immediate if the Hopf algebra $H$ is cotriangular, because in this case the braided antipode $\underline{S}$ is an algebra map. In the coquasitriangular case, with $A$ quasi-commutative, few more passages are needed. We evaluate the algebra map $f$ on

$$
\underline{S}(h \cdot k)=\circ \circ \Psi_{\underline{H}, \underline{H}}^{R}(\underline{S}(h) \otimes \underline{S}(k))=\underline{S}(k)_{(0)} \dot{\circ} \underline{S}(h)_{(0)} R\left(\underline{S}(h)_{(1)} \otimes \underline{S}(k)_{(1)}\right),
$$

$h, k \in \underline{H}$, this being the braided anti-algebra map property (2.22) of the braided antipode. We compute

$$
\begin{aligned}
\mathrm{f}(\underline{S}(h \cdot k)) & =\mathrm{f}\left(\underline{S}(k)_{(0)} \dot{S} \underline{S}(h)_{(0)}\right) R\left(\underline{S}(h)_{(1)} \otimes \underline{S}(k)_{(1)}\right) \\
& =\mathrm{f}\left(\underline{S}(k)_{(0)}\right) \mathrm{f}\left(\underline{S}(h)_{(0)}\right) R\left(\underline{S}(h)_{(1)} \otimes \underline{S}(k)_{(1)}\right) \\
& =\mathrm{f}(\underline{S}(k))_{(0)} \mathrm{f}(\underline{S}(h))_{(0)} R\left(\mathrm{f}(\underline{S}(h))_{(1)} \otimes \mathrm{f}(\underline{S}(k))_{(1)}\right) \\
& =\mathrm{f}(\underline{S}(h)) \mathrm{f}(\underline{S}(k))
\end{aligned}
$$

where for the last but one equality we used that $f$ is a morphism of comodules and for the last equality we used quasi-commutativity of the algebra $A$, as defined in (2.28). Therefore $\overline{\mathbf{f}}(h \cdot k)=\overline{\mathbf{f}}(h) \overline{\mathbf{f}}(k)$ as claimed.

In the commutative case, for a principal $G$-bundle $\pi: P \rightarrow M$ which is trivial, the gauge group is isomorphic to the group of maps from $M$ to $G$ (see e.g. [17, §7.1, Prop.1.7]). For Hopf-Galois extensions we have:

Lemma 3.3. Let $B \subseteq A$ be a trivial Hopf-Galois extension, with $(H, R)$ coquasitriangular and $A \in \mathcal{A}_{q c}^{(H, R)}$. Then $R$ is trivial, $H$ and $A$ are commutative, and the gauge group $\mathcal{G}_{A}$ coincides with the group (with convolution product $*$ ) of algebra maps from $H$ to $B$ :

$$
\mathcal{G}_{A} \simeq(\{\alpha: H \rightarrow \underset{19}{B \text { algebra maps }\}, *) .}
$$


Proof. For a trivial extension with $B$ in the centre of $A$, the cleaving map gives the isomorphism $A \simeq B \otimes H$ in $\mathcal{A}_{q c}^{(H, R)}$, with coaction $\delta=\mathrm{id} \otimes \Delta$. This implies that $H$ is quasicommutative, and therefore, cf. Example 2.21, $H$ is commutative with trivial $R$-form, so that $A$ is commutative as well. As for the gauge group $\mathcal{G}_{A}=\operatorname{Hom}_{\mathcal{A}^{H}}(\underline{H}, B \otimes H)$, observe first that the braided Hopf algebra $\underline{H}$ is isomorphic as a Hopf algebra to $H$. Indeed, since $R$ is trivial, the product in $\underline{H}$ equals that in $H$ and the braiding $\Psi_{H, H}^{R}$ is trivial. Next, each $\mathrm{f}: H \rightarrow B \otimes H$ in $\mathcal{G}_{A}$ determines an algebra map $\alpha_{\mathrm{f}}:=(\mathrm{id} \otimes \varepsilon) \circ \mathrm{f}: H \rightarrow B$. Conversely, with any algebra map $\alpha: H \rightarrow B$, one has a map $\mathrm{f}_{\alpha}:=(\alpha \otimes \mathrm{id}) \circ \mathrm{Ad}: H \rightarrow B \otimes H$ (cf. [3. Thm.5.4]). It is easy to verify that $\mathrm{f}_{\alpha}$ is a morphism of $H$-comodules:

$\left(\mathrm{f}_{\alpha} \otimes \mathrm{id}\right) \operatorname{Ad}(h)=\mathrm{f}_{\alpha}\left(h_{(2)}\right) \otimes S\left(h_{(1)}\right) h_{(3)}=\alpha\left(h_{(3)}\right) \otimes S\left(h_{(2)}\right) h_{(4)} \otimes S\left(h_{(1)}\right) h_{(5)}=(\mathrm{id} \otimes \Delta) \mathrm{f}_{\alpha}(h)$.

It is also an algebra map being a composition of such maps:

$$
\mathrm{f}_{\alpha}: H \stackrel{\mathrm{Ad}}{\longrightarrow} H \otimes H \stackrel{\alpha \otimes \mathrm{id}}{\longrightarrow} B \otimes H .
$$

One easily sees that $\alpha_{\mathrm{f}_{\alpha}}=\alpha$ and $\mathrm{f}_{\alpha_{\mathrm{f}}}=\mathrm{f}$, being $\mathrm{f}$ a comodule map, so that $(\mathrm{f} \otimes \mathrm{id}) \mathrm{Ad}=$ $(\mathrm{id} \otimes \Delta) \mathrm{f}$.

We note that while the algebras in a trivial Hopf-Galois extension $B \subseteq A \in \mathcal{A}_{q c}^{(H, R)}$ are commutative, for cleft Hopf-Galois extensions this need not be the case, and their gauge group is in general not given by $(\{\alpha: \underline{H} \rightarrow B$ algebra maps $\}, *)$. See Remark 4.18 later on.

Also for a principal $G$-bundle $\pi: P \rightarrow M$ with $G$ abelian the gauge group is isomorphic to the group of maps from $M$ to $G$. For Hopf-Galois extensions we have a similar result if the Hopf-algebra is cocommutative. A coquasitriangular Hopf algebra $(H, R)$ which is cocommutative is also commutative (cf. (2.7) and comments after Example 2.4). Nevertheless, since the $R$-form can be nontrivial, the algebra $A$ in the inclusion $B \subseteq A$ can be noncommutative. However the gauge group does not depend on $A$ :

Lemma 3.4. Let $(H, R)$ be a coquasitriangular and cocommutative Hopf algebra, and let $B=A^{c o H} \subseteq A \in \mathcal{A}_{q c}^{(H, R)}$ be a Hopf-Galois extension. Then its gauge group $\mathcal{G}_{A}$ coincides with the group of algebra maps from $H$ to $B$ with the convolution product $*$ :

$$
\mathcal{G}_{A} \simeq(\{\mathrm{f}: H \rightarrow B \text { algebra maps }\}, *) .
$$

Proof. Since $H$ is cocommutative, the adjoint coaction Ad is trivial, so the product in $\underline{H}$ equals that in $H$ (which is commutative due to coquasitriangularity) and the braiding $\Psi_{\underline{H}, \underline{H}}^{R}$ is trivial. Thus, the associated braided Hopf algebra $\underline{H}$ is isomorphic to $H$ as a Hopf algebra. Triviality of the adjoint coaction implies that each $H$-equivariant $\mathbb{K}$-linear map $\mathrm{f}: \underline{H} \rightarrow A$ satisfies $\delta \mathrm{f}(h)=\mathrm{f}(h) \otimes 1$, that is the image of $\mathrm{f}$ is contained in the subalgebra $B$ of coinvariants. In particular $H$-equivariant algebra maps $\mathrm{f} \in \mathcal{G}_{A}=\operatorname{Hom}_{\mathcal{A}^{H}}(\underline{H}, A)$ are algebra maps $\mathrm{f}: \underline{H} \rightarrow B$, then algebra maps $\mathrm{f}: H \rightarrow B$.

Example 3.5. The Hopf bundle. Consider the $\mathcal{O}(U(1))$-Hopf-Galois extension $\mathcal{O}\left(S^{2}\right) \subset$ $\mathcal{O}(S U(2))$. By Lemma 3.4 its gauge group is given by

$$
\mathcal{G}_{A}=\left(\left\{\mathrm{f}: \mathcal{O}(U(1)) \rightarrow \mathcal{O}\left(S^{2}\right) \text { algebra maps }\right\}, *\right) .
$$

Since $\mathcal{O}(U(1))$ is linearly spanned by group-like elements, the convolution product equals the point-wise product and we obtain $\mathcal{G}_{A} \simeq\left(\left\{\mathrm{f}: S^{2} \rightarrow U(1)\right\}, \cdot\right)$, as expected. 


\subsection{The gauge group of vertical automorphisms.}

For any Hopf-Galois extension $B \subseteq A \in \mathcal{A}_{q c}^{(H, R)}$ we show that $\operatorname{Aut}_{B}(A)$, defined as in the commutative case in (3.4), is a group. This uses properties of the (dual of the classical) translation map $\tau=t^{*}$ (cf. Example 2.2) leading to the following:

Proposition 3.6. Let $B=A^{c o H} \subseteq A$ be an $H$-Hopf-Galois extension with $(H, R)$ coquasitriangular and $A \in \mathcal{A}_{q c}^{(H, R)}$. The $\mathbb{K}$-module

$$
\operatorname{Aut}_{B}(A):=\operatorname{Hom}_{B} \mathcal{A}^{H}(A, A)=\left\{\mathrm{F} \in \operatorname{Hom}_{\mathcal{A}^{H}}(A, A) \mid \mathrm{F}_{\left.\right|_{B}}=\mathrm{id}\right\}
$$

of left $B$-module, right $H$-comodule algebra morphisms is a group with respect to the composition of maps

$$
\mathrm{F} \cdot \mathrm{G}:=\mathrm{G} \circ \mathrm{F}
$$

for all $\mathrm{F}, \mathrm{G} \in \operatorname{Aut}_{B}(A)$. For $\mathrm{F} \in \operatorname{Aut}_{B}(A)$ its inverse $\mathrm{F}^{-1} \in \operatorname{Aut}_{B}(A)$ is given by

$$
\begin{aligned}
\mathrm{F}^{-1}:=m \circ(\mathrm{id} \otimes m) \circ\left(\mathrm{id} \otimes F \bigotimes_{B} \mathrm{id}\right) \circ(\mathrm{id} \otimes \tau) \circ \underline{\delta}^{A}: A & \longrightarrow A \\
a & \longmapsto a_{(0)} \mathrm{F}\left(a_{(1)}^{<1>}\right) a_{(1)}^{<2>}
\end{aligned}
$$

where $\tau=\left.\chi^{-1}\right|_{1 \otimes \underline{H}}$ is the translation map.

Proof. The reversed composition order $\mathrm{F} \cdot \mathrm{G}=\mathrm{G} \circ \mathrm{F}$ stems from the contravariant property of the pull-back $\varphi \mapsto \mathrm{F}=\varphi^{*}$ used in the commutative case $A=\mathcal{O}(P)$. The expression for the inverse map $F^{-1}=\varphi^{*-1}$ is induced from that of $\varphi^{-1}$. The map $\mathrm{F}^{-1}$ is welldefined because $F \otimes_{B}$ id is well-defined due to the $B$-linearity of $\mathrm{F}$. We show $\mathrm{F}^{-1} \in$ $\operatorname{Aut}_{B}(A)$. Clearly $\mathrm{F}_{\left.\right|_{B}}^{-1}=\mathrm{id}$ since $\mathrm{F}$ and $\tau$ are unital; $\mathrm{F}^{-1}$ is an $H$-comodule algebra map because composition of $H$-comodule algebra maps (for the product $m_{A}: A \otimes A \rightarrow A$ see Proposition 2.18, for $m: A \bigotimes_{B} A \rightarrow A$ recall the proof concerning the canonical map $\chi$ in Theorem 2.29, for $\underline{\delta}^{A}: A \rightarrow A \otimes \underline{H}$ see Lemma 2.12$)$.

We recall the identity $a_{(0)} \tau\left(a_{(1)}\right)=\chi^{-1} \circ \chi\left(1 \otimes_{B} a\right)=1 \otimes_{B} a$, for all $a \in A$. To show that $\mathrm{F}^{-1} \circ \mathrm{F}=$ id we evaluate $\mathrm{F}^{-1}$, as from definition (3.7), on $F(a)$ and use that $F$ is $H$-equivariant and that it is an algebra map:

$$
\mathrm{F}^{-1}(\mathrm{~F}(a))=\mathrm{F}\left(a_{(0)}\right) \mathrm{F}\left(a_{(1)}^{<1>}\right) a_{(1)}^{<2>}=\mathrm{F}\left(a_{(0)} a_{(1)}^{<1>}\right) a_{(1)}^{<2>}=\mathrm{F}(1) a=a .
$$

To show that $\mathrm{F} \circ \mathrm{F}^{-1}=$ id requires property (2.34) of the translation map. Firstly, by applying $m \circ\left(\mathrm{id} \otimes_{B} \mathrm{~F}\right)$ to the identity $a_{(0)} \tau\left(a_{(1)}\right)=1 \otimes_{B} a$ we obtain

$$
\mathrm{F}(a)=a_{(0)} a_{(1)}^{<1>} \mathrm{F}\left(a_{(1)}^{<2>}\right) .
$$

Then we replace $a$ with $\mathrm{F}^{-1}(a)$, use that $\mathrm{F}^{-1}$ is a comodule map and obtain

$$
\left(\mathrm{F} \circ \mathrm{F}^{-1}\right)(a)=\mathrm{F}\left(\mathrm{F}^{-1}(a)\right)=a_{(0)} \mathrm{F}\left(a_{(1)}^{<1>}\right) a_{(1)}^{<2>} a_{(2)}^{<1>} \mathrm{F}\left(a_{(2)}^{<2>}\right) .
$$

Next, the quasi-commutativity of $A$ and the $H$-comodule algebra map property of $\mathrm{F}$ give

$$
\begin{aligned}
\mathrm{F}\left(\mathrm{F}^{-1}(a)\right) & =a_{(0)} a_{(1)}^{<2>}{ }_{(0)}^{<a_{(2)}<1>}{ }_{(0)} \mathrm{F}\left(a_{(1)}^{<1>}{ }_{(0)}\right) \mathrm{F}\left(a_{(2)}^{<2>}\right) R\left(a_{(1)}^{<1>} \otimes a_{(1)}^{<2>}{ }_{(1)}^{<a_{(2)}}{ }_{(1)}^{<1>}\right) \\
& =a_{(0)} a_{(1)}^{<2>}{ }_{(0)} a_{(2)}^{<1>}{ }_{(0)} \mathrm{F}\left(a_{(1)}^{<1>}{ }_{(0)}^{<a_{(2)}}{ }^{<>}\right) R\left(a_{(1)}^{<1>}{ }_{(1)} \otimes a_{(1)}^{<2>}{ }_{(1)}^{<a_{(2)}}{ }_{(1)}\right) .
\end{aligned}
$$

Finally, property (2.35) of the translation map implies

$$
\mathrm{F}\left(\mathrm{F}^{-1}(a)\right)=a_{(0)} \varepsilon\left(a_{(1)}\right)=a .
$$

This ends the proof. 
Remark 3.7. By definition, two Hopf-Galois extensions $A, A^{\prime} \in \mathcal{A}^{H}$ of a fixed algebra $B$ are isomorphic provided there exists an isomorphism of $H$-comodule algebras $A \rightarrow A^{\prime}$. This is the algebraic counterpart for noncommutative principal bundles of the geometric notion of isomorphism of principal $G$-bundles with fixed base space. As in the geometric case this notion is relevant in the homotopy classification of noncommutative principal bundles, see e.g. [19, §7.2]. In the coquasitriangular and quasi-commutative context of the present paper, if $A, A^{\prime} \in \mathcal{A}_{q c}^{(H, R)}$ are isomorphic via $\omega: A \rightarrow A^{\prime}$, then the groups $\operatorname{Aut}_{B}(A)$ and $\operatorname{Aut}_{B}\left(A^{\prime}\right)$ are isomorphic via

$$
\operatorname{Aut}_{B}(A) \rightarrow \operatorname{Aut}_{B}\left(A^{\prime}\right), \quad \mathrm{F} \mapsto \omega \circ \mathrm{F} \circ \omega^{-1} .
$$

Indeed, even if in general $\omega$ is not the identity on $B, \omega(B) \subseteq B$ being $\omega$ a morphism of $H$-comodules. Thus $\left.\omega \circ \mathrm{F} \circ \omega^{-1}\right|_{B}=\mathrm{id}$ and $\omega \circ \mathrm{F} \circ \omega^{-1} \in \operatorname{Aut}_{B}\left(A^{\prime}\right)$ as claimed. Therefore the gauge group $\mathcal{G}_{A}$ of an $H$-Hopf-Galois extension depends only on the isomorphism class of the extension, rather than on the single representative.

Example 3.8. Galois field extensions. Let $\mathbb{E}$ be a field, $\mathbb{K} \subseteq \mathbb{E}$ and $G=\left\{g_{i}\right\}$ a finite group acting on $\mathbb{E}$ as automorphisms of $\mathbb{E}$. Let $\mathbb{F} \supseteq \mathbb{K}$ be the fixed field of the $G$ action. By Artin's theorem if the $G$-action is faithful, $\mathbb{E}$ is a Galois extension of $\mathbb{F}$ and $G$ is its Galois group (the group of authomorphisms of $\mathbb{E}$ that leave $\mathbb{F}$ invariant). The $G$ action $a \mapsto g_{i}(a), a \in \mathbb{E}$, induces a coaction of the dual $(\mathbb{K} G)^{*}$ of the group algebra $\mathbb{K} G$, $\delta: \mathbb{E} \rightarrow \mathbb{E} \otimes(\mathbb{K} G)^{*}, a \mapsto \sum_{i} g_{i}(a) \otimes \beta_{i}$, where $\left\{\beta_{i}\right\}$ is the basis of $(\mathbb{K} G)^{*}$ dual to the basis $\left\{g_{i}\right\}$ of $\mathbb{K} G$. In [26, $\left.\S 8.1 .2\right]$ it is proven that $\mathbb{E}$ is a Galois field extension of $\mathbb{F}$ with Galois group $G$ if and only if the $\mathbb{K}$-algebra $\mathbb{E}$ is a Hopf-Galois extension of $\mathbb{F}=\mathbb{E}^{c o(\mathbb{K} G)^{*}}$. In this case consider the trivial coquasitriangular structure on $(\mathbb{K} G)^{*}$. The gauge group $\operatorname{Aut}_{\mathbb{F}}(\mathbb{E})$ consists of maps $\mathrm{F} \in G$ which are morphisms of $(\mathbb{K} G)^{*}$-comodules, $\delta \mathrm{F}=(\mathrm{F} \otimes \mathrm{id}) \delta$. This is equivalent to requiring $\mathrm{F} g_{i}=g_{i} \mathrm{~F}$ for each $i$. Thus $\mathrm{Aut}_{\mathbb{F}}(\mathbb{E})=Z(G)$, the center of the Galois group.

Example 3.9. Graded algebras. Let $G$ be a group, with neutral element $e$, and let $H=\mathbb{K} G$ be its group algebra. An algebra $A$ is $G$-graded, that is $A=\oplus_{g \in G} A_{g}$ and $A_{g} A_{h} \subseteq A_{g h}$ for all $g, h \in G$, if and only if $A$ is a right $\mathbb{K} G$-comodule algebra with coaction $\delta: A \rightarrow A \otimes \mathbb{K} G$, $a \mapsto \sum a_{g} \otimes g$ for $a=\sum a_{g}, a_{g} \in A_{g}$. Moreover, the algebra $A$ is strongly $G$-graded, that is $A_{g} A_{h}=A_{g h}$, if and only if $A_{e}=A^{c o(\mathbb{K} G)} \subseteq A$ is Hopf-Galois (see e.g. [26, Thm.8.1.7]). One can easily see that

$$
\operatorname{Hom}_{A_{e} \mathcal{A}^{\mathbb{K} G}}(A, A)=\left\{\mathrm{F}: A \rightarrow A \text { algebra maps }|\mathrm{F}|_{A_{e}}=\mathrm{id}, \mathrm{F}\left(A_{g}\right) \subseteq A_{g}\right\} .
$$

Let now $H=\mathbb{K} G$ be coquasitriangular and $A$ be quasi-commutative. Then Proposition 3.6 shows that $\operatorname{Hom}_{A_{e} \mathcal{A}^{\mathbb{K} G}}(A, A)$ is a group, the gauge group $\operatorname{Aut}_{A_{e}}(A)$ of the Hopf-Galois extension $A_{e} \subseteq A$. Notice that $H=\mathbb{K} G$ coquasitriangular implies $H$ commutative and hence $G$ abelian (cf. remark after Example 2.4). For $G=\mathbb{Z}$, with $H=\mathbb{C Z}=\mathcal{O}(U(1)$ ), the Hopf-Galois extension $A_{e} \subseteq A$ is a noncommutative principal $U(1)$-bundle. Examples with $G=\mathbb{Z}^{n}, H=\mathbb{C Z}^{n}=\mathcal{O}\left(\mathbb{T}^{n}\right)$, and $A_{e}=\mathbb{C}$ include Example 3.10 and Example 4.15 (noncommutative principal $U(1)^{n}$ bundles).

Example 3.10. Torus bundle over a point. (Its noncommutative deformation is in Example 4.15.) Let $\mathcal{O}\left(\mathbb{T}^{n}\right)$ be the commutative algebra of polynomial functions on the $n$-torus with generators $t_{j}, t_{j}^{*}$ satisfying $t_{j} t_{j}^{*}=1=t_{j}^{*} t_{j}$ (no sum on $j$ ) for $j=1, \ldots, n$. It is a $*$-Hopf algebra with costructures

$$
\Delta\left(t_{j}\right)=t_{j} \otimes t_{j}, \quad \varepsilon\left(t_{j}\right)=1, \quad S\left(t_{j}\right)=t_{j}^{*} .
$$

Hence $A=\mathcal{O}\left(\mathbb{T}^{n}\right)$, with coaction $\Delta: A \rightarrow A \otimes \mathcal{O}\left(\mathbb{T}^{n}\right)$, is a Hopf-Galois extension of $B=$ C. Vertical automorphisms $\mathbf{F} \in \operatorname{Aut}_{B}(A)=\operatorname{Hom}_{\mathcal{A}\left(\mathbb{T}^{n}\right)}(A, A)$ are determined by their 
action on the generators since they are algebra maps. In turn, $\mathcal{O}\left(\mathbb{T}^{n}\right)$-equivariance gives $\mathrm{F}\left(t_{i}\right)=(\varepsilon \otimes \mathrm{id}) \Delta\left(\mathrm{F}\left(t_{i}\right)\right)=\varepsilon\left(\mathrm{F}\left(t_{i}\right)\right) t_{i}$, and similarly for $t_{i}^{*}$, so that $\mathrm{F} \in \operatorname{Hom}_{\mathcal{A}^{\mathcal{O}\left(\mathbb{T}^{n}\right)}}(A, A)$ is determined by $\lambda_{i}=\varepsilon\left(F\left(t_{i}\right)\right)$ and $\lambda_{i}^{*}=\varepsilon\left(F\left(t_{i}^{*}\right)\right)$ with $\lambda_{i} \lambda_{i}^{*}=1$. Thus the gauge group is $\operatorname{Aut}_{B}(A) \simeq \mathbb{T}^{n}$. The result is in agreement with Lemma 3.4 which would give:

$$
\mathcal{G}_{A}=\left(\left\{f: \mathcal{O}\left(\mathbb{T}^{n}\right) \rightarrow \mathbb{C} \text { algebra maps }\right\}, *\right)
$$

that is, $\mathcal{G}_{A}$ as the set of characters of the algebra $\mathcal{O}\left(\mathbb{T}^{n}\right)$, hence $\mathcal{G}_{A}=\mathbb{T}^{n}$.

This example can be directly generalised to a $G$-bundle over a point, with $G$ any affine algebraic group, that is a subgroup of $G L(n, \mathbb{C})$.

\subsection{Equivalence of the gauge groups.}

We show the equivalence $\mathcal{G}_{A} \simeq \operatorname{Aut}_{B}(A)$.

Proposition 3.11. Let $(H, A)$ be a coquasitriangular Hopf algebra, and $B=A^{c o H} \subseteq A$ a Hopf-Galois extension, where $A \in \mathcal{A}_{q c}^{(H, R)}$ is a quasi-commutative H-comodule algebra. The groups $\left(\mathcal{G}_{A}, *\right)$ and $\left(\operatorname{Aut}_{B}(A), \cdot\right)$ are isomorphic via the map

$$
\begin{aligned}
\underline{\theta}_{A}: \mathcal{G}_{A} & \longrightarrow \operatorname{Aut}_{B}(A) \\
\mathrm{f} & \mapsto \mathrm{F}_{\mathrm{f}}:=m_{A} \circ\left(\operatorname{id}_{A} \otimes \mathrm{f}\right) \circ \underline{\delta}^{A} \quad: a \mapsto a_{(0)} \mathrm{f}\left(a_{(1)}\right),
\end{aligned}
$$

with inverse

$$
\mathrm{F} \mapsto \mathrm{f}_{\mathrm{F}}:=m_{A} \circ\left(\mathrm{id}_{A} \bigotimes_{B} \mathrm{~F}\right) \circ \tau \quad: h \mapsto h^{<1>} \mathrm{F}\left(h^{<2>}\right) .
$$

Proof. As mentioned after equation (3.4), without the requirement that elements of $\mathcal{G}_{A}$ and $\operatorname{Aut}_{B}(A)$ are algebra maps, the group isomorphism was proven in [3, §5] using the linear map $\theta_{A}: \mathrm{f} \mapsto \mathrm{F}_{\mathrm{f}}=m_{A} \circ\left(\mathrm{id}_{A} \otimes \mathrm{f}\right) \circ \delta^{A}$. When $A \in \mathcal{A}_{q c}^{(H, R)}$ we restrict to gauge transformations that are algebra maps. Since $\theta_{A}$ restricts as a linear map to $\underline{\theta}_{A}$ in (3.9) we just have to show that when $f$ is an algebra map, the corresponding $F_{f}$ is an algebra map and vice versa. This is so because $F_{f}$ is the composition of the algebra maps $m_{A}$, $\mathrm{id}_{A} \otimes \mathrm{f}$ and $\underline{\delta}^{A}$, and similarly, for $\mathrm{f}_{F}$.

Remark 3.12. When the Hopf algebra $H$ and the $H$-comodule algebra $A$ are both equipped with compatible $*$-structures, that is such that the coaction is a *-algebra map, the morphisms which constitute the gauge group $\mathcal{G}_{A} \simeq \operatorname{Aut}_{B}(A)$ will also be required to be compatible with the $*$-structures.

\section{Deformations By 2-COCYCles}

A general theory of Drinfeld-twist deformation of Hopf-Galois extensions was developed in [1]. We specialise this theory to coquasitriangular Hopf algebras (so that the canonical map is an algebra map) and study the corresponding gauge groups in the context of the theory presented in the previous section.

\subsection{Twisting comodule algebras and coalgebras by 2-cocycles.}

We first recall some relevant results from the general theory of 2-cocycle deformations of algebras and comodules [12, 14, 11]; we follow [1, §2.2].

Let $H=\left(H, m, 1_{H}, \Delta, \varepsilon, S\right)$ be a Hopf algebra.

Definition 4.1. A unital convolution invertible 2-cocycle, or simply a 2-cocycle, on $H$ is a $\mathbb{K}$-linear map $\gamma: H \otimes H \rightarrow \mathbb{K}$ which is unital, that is $\gamma(h \otimes 1)=\varepsilon(h)=\gamma(1 \otimes h)$, for all $h \in H$, invertible for the convolution product and satisfies the 2-cocycle condition

$$
\gamma\left(g_{(1)} \otimes h_{(1)}\right) \gamma\left(g_{(2)} h_{(2)} \otimes k\right)=\gamma\left(h_{(1)} \otimes k_{(1)}\right) \gamma\left(g \otimes h_{(2)} k_{(2)}\right),
$$

for all $g, h, k \in H$. 
For $\gamma$ a 2-cocycle, we denote by $\bar{\gamma}: H \otimes H \rightarrow \mathbb{K}$ its convolution inverse. The condition (4.1) can be equivalently written in terms of $\bar{\gamma}$ as

$$
\bar{\gamma}\left(g_{(1)} h_{(1)} \otimes k\right) \bar{\gamma}\left(g_{(2)} \otimes h_{(2)}\right)=\bar{\gamma}\left(g \otimes h_{(1)} k_{(1)}\right) \bar{\gamma}\left(h_{(2)} \otimes k_{(2)}\right),
$$

for all $g, h, k \in H$.

Given a 2-cocycle $\gamma$ on $H$, the map $m_{\gamma}:=\gamma * m * \bar{\gamma}$,

$$
m_{\gamma}(h \otimes k):=h \cdot_{\gamma} k:=\gamma\left(h_{(1)} \otimes k_{(1)}\right) h_{(2)} k_{(2)} \bar{\gamma}\left(h_{(3)} \otimes k_{(3)}\right),
$$

for $h, k \in H$, defines an associative product on (the $\mathbb{K}$-module underlying) $H$. The resulting algebra $H_{\gamma}:=\left(H, m_{\gamma}, 1_{H}\right)$ is a Hopf algebra with coproduct $\Delta$ and counit $\varepsilon$ that are those of $H$, and with antipode $S_{\gamma}:=u_{\gamma} * S * \bar{u}_{\gamma}$, where

$$
\begin{array}{ll}
u_{\gamma}: H \longrightarrow \mathbb{K}, \quad h \longmapsto \gamma\left(h_{(1)} \otimes S\left(h_{(2)}\right)\right), \\
\bar{u}_{\gamma}: H \longrightarrow \mathbb{K}, \quad h \longmapsto \bar{\gamma}\left(S\left(h_{(1)}\right) \otimes h_{(2)}\right),
\end{array}
$$

(one the convolution inverse of the other).

The passage from $H$ to $H_{\gamma}$ affects also the category $\mathcal{M}^{H}$ of (right) $H$-comodules. Since the comodule condition (1.1) only involves the coalgebra structure of $H$, and $H_{\gamma}$ coincides with $H$ as a coalgebra, any $H$-comodule $V \in \mathcal{M}^{H}$ with coaction $\delta^{V}$ is a right $H_{\gamma}$-comodule when $\delta^{V}$ is thought of as a map $\delta^{V}: V \rightarrow V \otimes H_{\gamma}$. When thinking of $V$ as an object in $\mathcal{M}^{H_{\gamma}}$ we denote it by $V_{\gamma}$ and the coaction by $\delta^{V_{\gamma}}: V_{\gamma} \rightarrow V_{\gamma} \otimes H_{\gamma}$. For the same reason, any morphism $\psi: V \rightarrow W$ in $\mathcal{M}^{H}$ can be thought as a morphism $\psi: V_{\gamma} \rightarrow W_{\gamma}$ in $\mathcal{M}^{H_{\gamma}}$. Indeed the (identity) functor

$$
\Gamma: \mathcal{M}^{H} \rightarrow \mathcal{M}^{H_{\gamma}}
$$

defined on objects by $\Gamma(V):=V_{\gamma}$ and on morphisms by $\Gamma(\psi):=\psi$, is an equivalence of categories. The convolution inverse $\bar{\gamma}$ twists back $H_{\gamma}$ to $\left(H_{\gamma}\right)_{\bar{\gamma}}=H$ and $V_{\gamma}$ to $\left(V_{\gamma}\right)_{\bar{\gamma}}=V$.

We denote by $\left(\mathcal{M}^{H_{\gamma}}, \otimes^{\gamma}\right)$ the monoidal category of comodules for the Hopf algebra $H_{\gamma}$. Explicitly, for all objects $V_{\gamma}, W_{\gamma} \in \mathcal{M}^{H_{\gamma}}$ (with coactions $\delta^{V_{\gamma}}$ and $\delta^{W_{\gamma}}$ ), the right $H^{\gamma}$-coaction on $V_{\gamma} \otimes^{\gamma} W_{\gamma}$ is given, following (1.2), by

$$
\delta^{V_{\gamma} \otimes^{\gamma} W_{\gamma}}: V_{\gamma} \otimes^{\gamma} W_{\gamma} \longrightarrow V_{\gamma} \otimes^{\gamma} W_{\gamma} \otimes H_{\gamma}, \quad v \otimes^{\gamma} w \longmapsto v_{(0)} \otimes^{\gamma} w_{(0)} \otimes v_{(1)} \cdot \gamma w_{(1)} .
$$

Proposition 4.2. The functor $\Gamma: \mathcal{M}^{H} \rightarrow \mathcal{M}^{H_{\gamma}}$ together with the natural isomorphism $\varphi: \otimes^{\gamma} \circ(\Gamma \times \Gamma) \Rightarrow \Gamma \circ \otimes$ given for objects $V, W \in \mathcal{M}^{H}$ by the isomorphism of $H_{\gamma}$-comodules

$$
\varphi_{V, W}: V_{\gamma} \otimes^{\gamma} W_{\gamma} \longrightarrow(V \otimes W)_{\gamma}, \quad v \otimes^{\gamma} w \longmapsto v_{(0)} \otimes w_{(0)} \bar{\gamma}\left(v_{(1)} \otimes w_{(1)}\right),
$$

is an equivalence between the monoidal categories $\left(\mathcal{M}^{H}, \otimes\right)$ and $\left(\mathcal{M}^{H_{\gamma}}, \otimes^{\gamma}\right)$.

The functor $\Gamma$ induces an equivalence of categories of comodule algebras

$$
\Gamma: \mathcal{A}^{H} \rightarrow \mathcal{A}^{H_{\gamma}}, \quad\left(A, m_{A}=\cdot, \eta_{A}, \delta^{A}\right) \mapsto\left(A_{\gamma}, m_{A_{\gamma}}=\bullet_{\gamma}, \eta_{A_{\gamma}}, \delta^{A_{\gamma}}\right)
$$

which is not the identity on objects any longer. Given an object $A \in \mathcal{A}^{H}$ with multiplication $m_{A}$ and unit $\eta_{A}$, in order for the coaction $\delta^{A_{\gamma}}$ to be an algebra map one has to define a new product on $A_{\gamma}=\Gamma(A)$. The new algebra structure $m_{A_{\gamma}}, \eta_{A_{\gamma}}$ on $A_{\gamma} \in \mathcal{A}^{H_{\gamma}}$ is defined by using the components $\varphi_{-,-}$in (4.7) of the natural isomorphism $\varphi$, and by requiring the commutativity of the diagrams
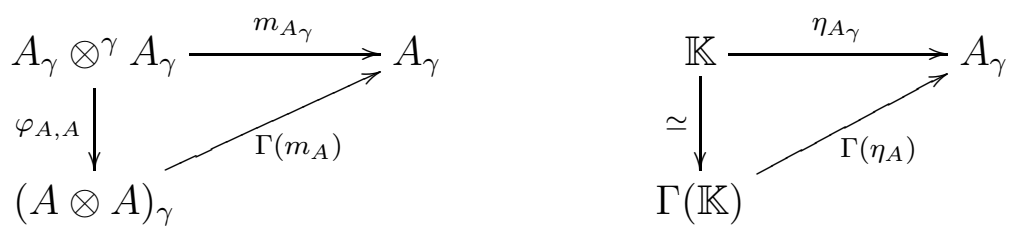
in the category $\mathcal{M}^{H_{\gamma}}$. Explicitly we have $\eta_{A_{\gamma}}=\eta_{A}$ and the deformed product reads as

$$
m_{A_{\gamma}}: A_{\gamma} \otimes^{\gamma} A_{\gamma} \longrightarrow A_{\gamma}, \quad a \otimes^{\gamma} a^{\prime} \longmapsto a \bullet_{\gamma} a^{\prime}:=a_{(0)} a_{(0)}^{\prime} \bar{\gamma}\left(a_{(1)} \otimes a_{(1)}^{\prime}\right) \text {. }
$$

Moreover, for any $\mathcal{A}^{H}$-morphism $\psi: A \rightarrow A^{\prime}$ one checks that $\Gamma(\psi)=\psi: A_{\gamma} \rightarrow A_{\gamma}^{\prime}$ is a morphism in $\mathcal{A}^{H_{\gamma}}$. With similar constructions, for $A, C \in \mathcal{A}^{H}$, one obtains equivalences

$$
\Gamma:{ }_{A} \mathcal{M}^{H} \rightarrow{ }_{A_{\gamma}} \mathcal{M}^{H_{\gamma}}, \quad \Gamma: \mathcal{M}_{C}{ }^{H} \rightarrow \mathcal{M}_{C_{\gamma}}{ }^{H_{\gamma}}, \quad \Gamma:{ }_{A} \mathcal{M}_{C}{ }^{H} \rightarrow{ }_{A_{\gamma}} \mathcal{M}_{C_{\gamma}}{ }^{H_{\gamma}}
$$

for the categories of relative Hopf-modules.

The functor $\Gamma$ also induces an equivalence of categories of comodule coalgebras

$$
\Gamma: \mathcal{C}^{H} \rightarrow \mathcal{C}^{H_{\gamma}}, \quad\left(C, \Delta_{C}, \varepsilon_{C}, \delta^{C}\right) \mapsto\left(C_{\gamma}, \Delta_{C_{\gamma}}, \varepsilon_{C_{\gamma}}, \delta^{C_{\gamma}}\right) .
$$

Each $H$-comodule coalgebra $C$ with co-structures $\left(\Delta_{C}, \varepsilon_{C}\right)$ is mapped to the $H_{\gamma}$-comodule coalgebra $C_{\gamma}=\Gamma(C)$ with co-structures $\left(\Delta_{C_{\gamma}}, \varepsilon_{C_{\gamma}}\right)$ defined by the commutativity of the diagrams
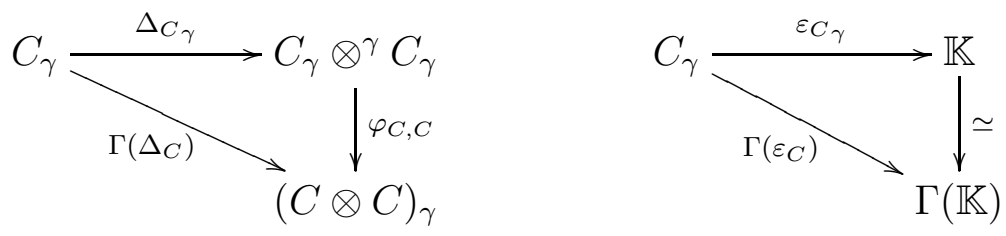

in the category $\mathcal{M}^{H_{\gamma}}$. The deformed coproduct explicitly reads

$$
\Delta_{C \gamma}: C_{\gamma} \longrightarrow C_{\gamma} \otimes^{\gamma} C_{\gamma}, c \longmapsto\left(c_{(1)}\right)_{(0)} \otimes^{\gamma}\left(c_{(2)}\right)_{(0)} \gamma\left(\left(c_{(1)}\right)_{(1)} \otimes\left(c_{(2)}\right)_{(1)}\right),
$$

while $\varepsilon_{C \gamma}=\varepsilon_{C}$. As before, $\Gamma$ acts as the identity on morphisms.

Example 4.3. The right $H$-comodule $\underline{H}=(H, \mathrm{Ad})$ is a comodule coalgebra with coproduct and counit those of the Hopf algebra $H, \Delta_{\underline{H}}=\Delta_{H}$ and $\varepsilon_{\underline{H}}=\varepsilon_{H}$. Its twist deformation $\underline{H}_{\gamma}:=\left(\Gamma(\underline{H}), \Delta_{\underline{H}_{\gamma}}, \varepsilon_{\underline{H}_{\gamma}}, \mathrm{Ad}\right)$ is an $H_{\gamma}$-comodule coalgebra. Explicitly the coproduct (4.12) of an element $h \in \underline{H}_{\gamma}$ reads $\Delta_{\underline{H}_{\gamma}}(h)=h_{(2)} \otimes^{\gamma} h_{(5)} \gamma\left(S\left(h_{(1)}\right) h_{(3)} \otimes S\left(h_{(4)}\right) h_{(6)}\right)$.

On the other hand, given a twist $\gamma$ on $H$, we have a second $H_{\gamma}$-comodule coalgebra. It is given by the right $H_{\gamma}$-comodule $\underline{H_{\gamma}}=\left(H_{\gamma}, \mathrm{Ad}_{\gamma}\right)$ with coaction

$$
\operatorname{Ad}_{\gamma}: \underline{H_{\gamma}} \longrightarrow \underline{H_{\gamma}} \otimes H_{\gamma}, \quad h \longmapsto h_{(2)} \otimes^{\gamma} S_{\gamma}\left(h_{(1)}\right){ }_{\gamma} h_{(3)}
$$

and coproduct and counit those of the twisted Hopf algebra $H_{\gamma}$, that is, those of $H$ : $\Delta_{\underline{H_{\gamma}}}=\Delta_{H}$ and $\varepsilon_{\underline{H_{\gamma}}}=\varepsilon_{H}$.

We recall from [1, Thm.3.4] that the comodule coalgebras $\underline{H}_{\gamma}$ and $\underline{H}_{\gamma}$ are isomorphic:

Theorem 4.4. The $\mathbb{K}$-linear map

$$
\mathcal{Q}: \underline{H_{\gamma}} \longrightarrow \underline{H}_{\gamma}, \quad h \longmapsto h_{(3)} u_{\gamma}\left(h_{(1)}\right) \bar{\gamma}\left(S\left(h_{(2)}\right) \otimes h_{(4)}\right)
$$

is an isomorphism of right $H_{\gamma}$-comodule coalgebras, with inverse

$$
\mathcal{Q}^{-1}: \underline{H}_{\gamma} \longrightarrow \underline{H_{\gamma}}, \quad h \longmapsto h_{(3)} \bar{u}_{\gamma}\left(h_{(2)}\right) \gamma\left(S\left(h_{(1)}\right) \otimes h_{(4)}\right) .
$$

\subsection{The coquasitriangular case.}

In this section we consider 2-cocycles on coquasitriangular Hopf algebras and study twisted associated bialgebras.

Recall that if $H$ is coquasitriangular, the category $\mathcal{A}^{H}$ is monoidal (see Proposition 2.9). Also, as mentioned in Example 2.7, if $R$ is the universal $R$-form of $H$, the twisted Hopf algebra $H_{\gamma}$ is coquasitriangular with universal $R$-form $R_{\gamma}=\gamma_{21} * R * \bar{\gamma}$. 
Proposition 4.5. Let $(H, R)$ be a coquasitriangular Hopf algebra and $\gamma$ a 2-cocycle on $H$. There is an equivalence of monoidal categories between $\left(\mathcal{A}^{H}, \nabla\right)$ and $\left(\mathcal{A}^{H_{\gamma}}, \nabla^{\gamma}\right)$ given by the functor $\Gamma: \mathcal{A}^{H} \rightarrow \mathcal{A}^{H_{\gamma}}$ in (4.8) and the isomorphisms in $\mathcal{A}^{H_{\gamma}}$

$$
\varphi_{A, C}: A_{\gamma} \otimes^{\gamma} C_{\gamma} \longrightarrow(A \otimes C)_{\gamma}, \quad a \nabla^{\gamma} c \longmapsto a_{(0)} \otimes c_{(0)} \bar{\gamma}\left(a_{(1)} \otimes c_{(1)}\right),
$$

with $A_{\gamma} \otimes^{\gamma} C_{\gamma}$ the braided tensor product of the algebras $A_{\gamma}$ and $C_{\gamma}$, and $(A \otimes C)_{\gamma}$ the image via $\Gamma$ of the braided tensor product of the algebras $A$ and $C$. (Cf. Proposition 4.2.)

Proof. Due to Proposition 4.2 we just need to prove that the isomorphisms $\varphi_{A, C}$ in $\mathcal{M}^{H_{\gamma}}$ are also algebra maps: $\varphi_{A, C} \circ m_{A_{\gamma} \bigotimes \gamma C_{\gamma}}=m_{(A \bowtie C)_{\gamma}} \circ\left(\varphi_{A, C} \otimes \varphi_{A, C}\right)$, that is,

$$
\varphi_{A, C}\left(\left(a \nabla^{\gamma} c\right) \cdot\left(a^{\prime} \nabla^{\gamma} c^{\prime}\right)\right)=\varphi_{A, C}\left(a \nabla^{\gamma} c\right) \boldsymbol{-}_{\gamma} \varphi_{A, C}\left(a^{\prime} \nabla^{\gamma} c^{\prime}\right),
$$

for all $a, a^{\prime}, \in A_{\gamma}, c, c^{\prime} \in C_{\gamma}$. Here the --product on the l.h.s. is the product in the braided tensor product algebra $A_{\gamma} \nabla^{\gamma} C_{\gamma}$ (defined in (2.15) ), while the $\boldsymbol{r}_{\gamma}$-product on the r.h.s. is the twist deformation (as in (4.9)) of the - -product in the tensor product algebra $A \otimes C$.

We prove (4.15) by first evaluating it on specific products and then using the associativity of the multiplications $\cdot$ and $\boldsymbol{\cdot} \gamma$. Firstly we show that

$$
\varphi_{A, C}\left(\left(a \nabla^{\gamma} 1_{C}\right) \cdot\left(a^{\prime} \nabla^{\gamma} c^{\prime}\right)\right)=\varphi_{A, C}\left(a \nabla^{\gamma} 1_{C}\right) \cdot{ }_{\gamma} \varphi_{A, C}\left(a^{\prime} \otimes^{\gamma} c^{\prime}\right) .
$$

Explicitly

$$
\begin{aligned}
& \left.\varphi_{A, C}\left(\left(a \nabla^{\gamma} 1_{C}\right) \cdot\left(a^{\prime} \nabla^{\gamma} c^{\prime}\right)\right)=\varphi_{A, C}\left(\left(a \bullet_{\gamma} a^{\prime}\right) \nabla^{\gamma} c^{\prime}\right)\right) \\
& =\varphi_{A, C}\left(\left(a_{(0)} a_{(0)}^{\prime}\right) \otimes^{\gamma} c^{\prime}\right) \bar{\gamma}\left(a_{(1)} \otimes a_{(1)}^{\prime}\right) \\
& =a_{(0)} a_{(0)}^{\prime} \otimes c^{\prime}{ }_{(0)} \bar{\gamma}\left(a_{(1)} a_{(1)}^{\prime} \otimes c_{(1)}\right) \bar{\gamma}\left(a_{(2)} \otimes a_{(2)}^{\prime}\right) \\
& =a_{(0)} a_{(0)}^{\prime} \otimes c_{(0)}^{\prime} \bar{\gamma}\left(a_{(1)} \otimes a_{(1)}^{\prime} c_{(1)}^{\prime}\right) \bar{\gamma}\left(a_{(2)}^{\prime} \otimes c_{(2)}^{\prime}\right) \\
& =\left(a \otimes 1_{C}\right) \cdot{ }_{\gamma}\left(a_{(0)}^{\prime} \otimes c^{\prime}{ }_{(0)}\right) \bar{\gamma}\left(a_{(1)}^{\prime} \otimes c_{(1)}^{\prime}\right) \\
& =\left(a \otimes 1_{C}\right) \boldsymbol{\bullet}_{\gamma} \varphi_{A, C}\left(a^{\prime} \nabla^{\gamma} c^{\prime}\right) \\
& =\varphi_{A, C}\left(a \otimes^{\gamma} 1_{C}\right) \bullet_{\gamma} \varphi_{A, C}\left(a^{\prime} \otimes^{\gamma} c^{\prime}\right)
\end{aligned}
$$

having also used the 2-cocycle condition (4.2) for the fourth and fifth equalities. Since $\varphi_{A, C}\left(a \nabla^{\gamma} 1_{C}\right)=a \otimes 1_{C}$, the identity (4.16) just expresses the fact that $\varphi_{A, C}$ are isomorphisms in $A_{\gamma} \mathcal{M}$ for the obvious left action of $A$ and $A_{\gamma}$ on $A \otimes C$ and $A_{\gamma} \nabla^{\gamma} C_{\gamma}$ respectively, see (4.10). Similarly, since the $\varphi_{A, C}$ are isomorphisms in $\mathcal{M}_{C_{\gamma}}$, we have

$$
\varphi_{A, C}\left(\left(a \nabla^{\gamma} c\right) \cdot\left(1_{A} \nabla^{\gamma} c^{\prime}\right)\right)=\varphi_{A, C}\left(a \nabla^{\gamma} c\right) \boldsymbol{-}_{\gamma} \varphi_{A, C}\left(1_{A} \nabla^{\gamma} c^{\prime}\right) .
$$

Finally we have

$$
\begin{aligned}
\varphi_{A, C}\left(\left(1_{A} \otimes^{\gamma} c\right) \cdot\left(a^{\prime} \nabla^{\gamma} 1_{C}\right)\right) & =\varphi_{A, C}\left(a_{(0)}^{\prime} \otimes^{\gamma} c_{(0)}\right) R_{\gamma}\left(c_{(1)} \otimes a_{(1)}^{\prime}\right) \\
& =a_{(0)}^{\prime} \otimes c_{(0)} \bar{\gamma}\left(a_{(1)}^{\prime} \otimes c_{(1)}\right) R_{\gamma}\left(c_{(2)} \otimes a_{(2)}^{\prime}\right) \\
& =a_{(0)}^{\prime} \otimes c_{(0)} R\left(c_{(1)} \otimes a_{(1)}^{\prime}\right) \bar{\gamma}\left(c_{(2)} \otimes a_{(2)}\right) \\
& =\left(1_{A} \otimes c\right)_{(0)} \cdot\left(a^{\prime} \otimes 1_{C}\right)_{(0)} \bar{\gamma}\left(c_{(1)} \otimes a_{(1)}\right) \\
& =\varphi_{A, C}\left(1_{A} \otimes^{\gamma} c\right) \boldsymbol{-}_{\gamma} \varphi_{A, C}\left(a^{\prime} \otimes^{\gamma} 1_{C}\right)
\end{aligned}
$$

where in the third line we used that $R_{\gamma}=\gamma_{21} * R * \bar{\gamma}$ (cf. (2.9)). Thus on the generic product of two elements $\left(a \nabla^{\gamma} c\right)=\left(a \nabla^{\gamma} 1\right) \cdot\left(1 \nabla^{\gamma} c\right)$ and $\left(a^{\prime} \nabla^{\gamma} c^{\prime}\right)=\left(a^{\prime} \otimes^{\gamma} 1\right) \cdot\left(1 \nabla^{\gamma} c^{\prime}\right)$ the map $\varphi_{A, C}$ is an algebra map.

This result and Corollary 2.33 lead to 
Corollary 4.6. Let $(H, R)$ be a cotriangular Hopf algebra. With the notations of Proposition 4.5 , the restriction of the functor $(\Gamma, \varphi):\left(\mathcal{A}^{H}, \nabla\right) \rightarrow\left(\mathcal{A}^{H_{\gamma}}, \nabla^{\gamma}\right)$ to the subcategory $\left(\mathcal{A}_{q c}^{(H, R)}, \bigotimes\right)$ of quasi-commutative comodule algebras induces an equivalence of monoidal categories $\left(\mathcal{A}_{q c}^{(H, R)}, \bigotimes\right) \simeq\left(\mathcal{A}_{q c}^{\left(H_{\gamma}, R_{\gamma}\right)}, \nabla^{\gamma}\right)$.

In the context of coquasitriangular Hopf algebras in addition to the twist deformation of comodule algebras and comodule coalgebras (considered in 44.1 ) one next deforms braided bialgebras associated with $H$ (see Definition 2.13).

Proposition 4.7. Let $\left(L, m_{L}, \eta_{L}, \Delta_{L}, \varepsilon_{L}, \delta^{L}\right)$ be a braided bialgebra associated with a coquasitriangular Hopf algebra $(H, R)$, and $\gamma$ a 2-cocycle on $H$. The twist deformation of $\left(L, m_{L}, \eta_{L}, \delta^{L}\right)$ as an $H$-comodule algebra and of $\left(L, \Delta_{L}, \varepsilon_{L}, \delta^{L}\right)$ as an $H$-comodule coalgebra gives the braided bialgebra $\left(L_{\gamma}, m_{L_{\gamma}}, \eta_{L_{\gamma}}, \Delta_{L_{\gamma}}, \varepsilon_{L_{\gamma}}, \delta^{L_{\gamma}}\right)$ associated with the twisted Hopf algebra $H_{\gamma}$. Thus, $L_{\gamma}$ is a bialgebra in the braided monoidal category $\left(\mathcal{M}^{H_{\gamma}}, \otimes^{\gamma}, \Psi^{R_{\gamma}}\right)$ of $H_{\gamma}$-comodules. Moreover, if $L$ is a braided Hopf algebra, then $L_{\gamma}$ is a braided Hopf algebra with antipode $S_{L_{\gamma}}=\Gamma\left(S_{L}\right), \ell \mapsto S_{L_{\gamma}}(\ell)=S_{L}(\ell)$.

Proof. By the general theory, the $H_{\gamma}$-comodule $L_{\gamma}=\Gamma(L)$ is an $H_{\gamma}$-comodule algebra with unit $1_{L_{\gamma}}=1_{L}$ (or $\eta_{L_{\gamma}}=\Gamma\left(\eta_{L}\right)$ ) and deformed product $m_{L_{\gamma}}=\Gamma\left(m_{L}\right) \circ \varphi_{L, L}$ given by (4.9). Moreover $L_{\gamma}$ is an $H_{\gamma}$-comodule coalgebra with counit $\varepsilon_{L_{\gamma}}=\Gamma\left(\varepsilon_{L}\right), \ell \mapsto$ $\varepsilon_{L_{\gamma}}(\ell)=\varepsilon_{L}(\ell)$, and deformed coproduct $\Delta_{L_{\gamma}}=\varphi_{L, L}^{-1} \circ \Gamma\left(\Delta_{L}\right)$ given by (4.12). In order for $\left(L_{\gamma}, m_{L_{\gamma}}, \eta_{L_{\gamma}}, \Delta_{L_{\gamma}}, \varepsilon_{L_{\gamma}}, \delta^{L_{\gamma}}\right)$ to be a braided bialgebra associated with the twisted Hopf algebra $H_{\gamma}$ it suffices to show (cf. Definition 2.13) that $\Delta_{L_{\gamma}}$ is an algebra map for the product $m_{\gamma}$ in $L_{\gamma}$ and the product $m_{L_{\gamma} \otimes^{\gamma} L_{\gamma}}=\left(m_{L_{\gamma}} \otimes^{\gamma} m_{L_{\gamma}}\right) \circ\left(\operatorname{id}_{L_{\gamma}} \otimes^{\gamma} \Psi_{L_{\gamma}, L_{\gamma}}^{R_{\gamma}} \otimes^{\gamma} \operatorname{id}_{L_{\gamma}}\right)$ in $L_{\gamma} \otimes^{\gamma} L_{\gamma}$ :

$$
\Delta_{L_{\gamma}} \circ m_{L_{\gamma}}=m_{L_{\gamma} \boxplus \gamma L_{\gamma}} \circ\left(\Delta_{L_{\gamma}} \otimes^{\gamma} \Delta_{L_{\gamma}}\right) .
$$

On the one hand,

$$
\begin{aligned}
\Delta_{L_{\gamma}} \circ m_{L_{\gamma}} & =\varphi_{L, L}^{-1} \circ \Gamma\left(\Delta_{L}\right) \circ \Gamma\left(m_{L}\right) \circ \varphi_{L, L}=\varphi_{L, L}^{-1} \circ \Gamma\left(\Delta_{L} \circ m_{L}\right) \circ \varphi_{L, L} \\
& =\varphi_{L, L}^{-1} \circ \Gamma\left(m_{L \otimes L} \circ\left(\Delta_{L} \otimes \Delta_{L}\right)\right) \circ \varphi_{L, L}=\varphi_{L, L}^{-1} \circ \Gamma\left(m_{L \otimes L}\right) \circ \Gamma\left(\Delta_{L} \otimes \Delta_{L}\right) \circ \varphi_{L, L}
\end{aligned}
$$

where we have used that $L$ is a braided bialgebra associated with $H$ (cf. (2.20)).

On the other hand, the maps $\varphi_{-,-}$satisfy

$$
\Gamma\left(\Delta_{L} \otimes \Delta_{L}\right) \circ \varphi_{L, L}=\varphi_{L \bowtie L, L \bowtie L} \circ\left(\Gamma\left(\Delta_{L}\right) \otimes^{\gamma} \Gamma\left(\Delta_{L}\right)\right)
$$

as it can be verified on generic elements in $L_{\gamma} \nabla^{\gamma} L_{\gamma}$ by using that $L$ is an $H$-comodule coalgebra (cf. equation (1.3) ). Thus,

$$
\begin{aligned}
\Delta_{L_{\gamma}} \circ m_{L_{\gamma}} & =\varphi_{L, L}^{-1} \circ \Gamma\left(m_{L \otimes L}\right) \circ \varphi_{L \otimes L, L \bigotimes L} \circ\left(\Gamma\left(\Delta_{L}\right) \otimes^{\gamma} \Gamma\left(\Delta_{L}\right)\right) \\
& =\varphi_{L, L}^{-1} \circ m_{(L \otimes L)_{\gamma}} \circ\left(\Gamma\left(\Delta_{L}\right) \otimes^{\gamma} \Gamma\left(\Delta_{L}\right)\right) \\
& =m_{L_{\gamma} \otimes \gamma} L_{\gamma} \circ\left(\varphi_{L, L}^{-1} \otimes^{\gamma} \varphi_{L, L}^{-1}\right) \circ\left(\Gamma\left(\Delta_{L}\right) \otimes^{\gamma} \Gamma\left(\Delta_{L}\right)\right) \\
& =m_{L_{\gamma} \otimes \gamma L_{\gamma}} \circ\left(\Delta_{L_{\gamma}} \otimes^{\gamma} \Delta_{L_{\gamma}}\right),
\end{aligned}
$$

by using in the third equality that $\varphi_{-,-}$are algebra maps (cf. (4.15)).

If in addition $L$ has an antipode $S_{L}$ (by assumption an $H$-comodule map), its image under $\Gamma, S_{L_{\gamma}}:=\Gamma\left(S_{L}\right), \ell \mapsto S_{L_{\gamma}}(\ell)=S_{L}(\ell)$, is an $H_{\gamma}$-comodule map. We show it is an antipode for the twisted bialgebra $L_{\gamma}$. One easily verifies the equality

$$
\varphi_{L, L} \circ\left(\Gamma\left(\mathrm{id}_{L}\right) \otimes^{\gamma} \Gamma\left(S_{L}\right)\right) \circ \varphi_{L, L}^{-1}=\Gamma\left(\mathrm{id}_{L} \otimes S_{L}\right)
$$


of $H_{\gamma}$-equivariant maps. Then

$$
\begin{aligned}
m_{L_{\gamma}} \circ\left(\operatorname{id}_{L_{\gamma}} \otimes S_{L_{\gamma}}\right) \circ \Delta_{L_{\gamma}} & =\Gamma\left(m_{L}\right) \circ \varphi_{L, L} \circ\left(\Gamma\left(\mathrm{id}_{L}\right) \otimes \Gamma\left(S_{L}\right)\right) \circ \varphi_{L, L}^{-1} \circ \Gamma\left(\Delta_{L}\right) \\
& =\Gamma\left(m_{L}\right) \circ \Gamma\left(\mathrm{id}_{L} \otimes S_{L}\right) \circ \Gamma\left(\Delta_{L}\right) \\
& =\Gamma\left(m_{L} \circ\left(\mathrm{id}_{L} \otimes S_{L}\right) \circ \Delta_{L}\right) \\
& =\Gamma\left(\eta_{L} \circ \varepsilon_{L}\right)=\Gamma\left(\eta_{L}\right) \circ \Gamma\left(\varepsilon_{L}\right) \\
& =\eta_{L_{\gamma}} \circ \varepsilon_{L_{\gamma}} .
\end{aligned}
$$

Analogously one shows that $m_{L_{\gamma}} \circ\left(S_{L_{\gamma}} \otimes^{\gamma} \mathrm{id}_{L_{\gamma}}\right) \circ \Delta_{L_{\gamma}}=\eta_{L_{\gamma}} \circ \varepsilon_{L_{\gamma}}$.

Example 4.8. Let $\underline{H}$ be the braided Hopf algebra associated with a coquasitriangular Hopf algebra $(H, R)$ (cf. Example 2.14). Given a 2-cocycle $\gamma$ on $H$, by Proposition 4.7 we have the braided Hopf algebra $\underline{H}_{\gamma}=\left(\Gamma(\underline{H}), \dot{-}_{\gamma}, \Delta_{\underline{H}_{\gamma}}, \varepsilon_{\underline{H}_{\gamma}}, \eta_{\underline{H}_{\gamma}}, S_{\underline{H}_{\gamma}}\right.$, Ad) associated with the twisted Hopf algebra $H_{\gamma}$. It is given by the $H_{\gamma}$-comodule coalgebra $\underline{H}_{\gamma}$ of Example 4.3 endowed with the product

$$
h \cdot{ }_{\gamma} k=h_{(0)} \cdot k_{(0)} \bar{\gamma}\left(h_{(1)} \otimes k_{(1)}\right)=h_{(2)} \cdot k_{(2)} \bar{\gamma}\left(S\left(h_{(1)}\right) h_{(3)} \otimes S\left(k_{(1)}\right) k_{(3)}\right) .
$$

This is the twist deformation of the product $h \cdot k:=h_{(2)} k_{(2)} R\left(S\left(h_{(1)}\right) h_{(3)} \otimes S\left(k_{(1)}\right)\right)$ in $\underline{H}$ defined by (2.17). In terms of the product in $H$, the product $h \cdot{ }_{\gamma} k$ is written as

$$
h \cdot{ }_{\gamma} k=h_{(3)} k_{(3)} R\left(S\left(h_{(2)}\right) h_{(4)} \otimes S\left(k_{(2)}\right)\right) \bar{\gamma}\left(S\left(h_{(1)}\right) h_{(5)} \otimes S\left(k_{(1)}\right) k_{(4)}\right) .
$$

In addition to the braided Hopf algebra $\underline{H}_{\gamma}$ there is also the braided Hopf algebra $H_{\gamma}$ of the coquasitriangular Hopf algebra $\left(H_{\gamma}, R_{\gamma}\right)$ (cf. Example 2.14). The product in $\overline{H_{\gamma}}$ is as in (2.17): for all $h, k \in H_{\gamma}$, one has

$$
h_{\underline{\gamma}} k:=h_{(2)} \cdot \gamma k_{(2)} R_{\gamma}\left(S_{\gamma}\left(h_{(1)}\right) \cdot{ }_{\gamma} h_{(3)} \otimes S_{\gamma}\left(k_{(1)}\right)\right) .
$$

Recalling the product and antipode in $H_{\gamma}: h \cdot_{\gamma} k=\gamma\left(h_{(1)} \otimes k_{(1)}\right) h_{(2)} k_{(2)} \bar{\gamma}\left(h_{(3)} \otimes k_{(3)}\right)$, and $S_{\gamma}=u_{\gamma} * S * \bar{u}_{\gamma}$, we can rewrite $h \cdot \gamma k$ as

$$
\begin{aligned}
h_{\underline{\gamma}} k= & u_{\gamma}\left(h_{(1)}\right) u_{\gamma}\left(k_{(1)}\right) \bar{u}_{\gamma}\left(h_{(7)}\right) \bar{u}_{\gamma}\left(k_{(5)}\right) \gamma\left(h_{(8)} \otimes k_{(6)}\right) h_{(9)} k_{(7)} \\
& \bar{\gamma}\left(h_{(10)} \otimes k_{(8)}\right) \gamma\left(S\left(h_{(6)}\right) \otimes h_{(11)}\right) \gamma\left(S\left(k_{(4)}\right) \otimes S\left(h_{(5)}\right) h_{(12)}\right) \\
& R\left(S\left(h_{(4)}\right) h_{(13)} \otimes S\left(k_{(3)}\right)\right) \bar{\gamma}\left(S\left(h_{(3)}\right) h_{(14)} \otimes S\left(k_{(2)}\right)\right) \bar{\gamma}\left(S\left(h_{(2)}\right) \otimes h_{(15)}\right) .
\end{aligned}
$$

The braided Hopf algebras $\underline{H}_{\gamma}$ and $\underline{H}_{\gamma}$ are isomorphic:

Theorem 4.9. The $\mathbb{K}$-linear map $\mathcal{Q}: H_{\gamma} \longrightarrow \underline{H}_{\gamma}$ in (4.13) with inverse in (4.14) is an isomorphism of braided Hopf algebras associated with $H_{\gamma}$.

Proof. We know from Theorem 4.4 that the map $\mathcal{Q}$ is an isomorphism of $H_{\gamma}$-comodule coalgebras. We are left to show that $\mathcal{Q}$ is an algebra morphism. It maps the unit of $H_{\gamma}$ to the unit of $\underline{H}_{\gamma}$. In $\underline{H}_{\gamma}$ the product is given by formula (4.19). Thus we have

$$
\begin{aligned}
\mathcal{Q}(h) \dot{\gamma}_{\gamma} \mathcal{Q}(k) & =u_{\gamma}\left(h_{(1)}\right) u_{\gamma}\left(k_{(1)}\right) h_{(3)} \dot{ }_{\gamma} k_{(3)} \bar{\gamma}\left(S\left(h_{(2)}\right) \otimes h_{(4)}\right) \bar{\gamma}\left(S\left(k_{(2)}\right) \otimes k_{(4)}\right) \\
= & u_{\gamma}\left(h_{(1)}\right) u_{\gamma}\left(k_{(1)}\right) h_{(5)} k_{(5)} R\left(S\left(h_{(4)}\right) h_{(6)} \otimes S\left(k_{(4)}\right)\right) \bar{\gamma}\left(S\left(h_{(2)}\right) \otimes h_{(8)}\right) \\
& \bar{\gamma}\left(S\left(h_{(3)}\right) h_{(7)} \otimes S\left(k_{(3)}\right) k_{(6)}\right) \bar{\gamma}\left(S\left(k_{(2)}\right) \otimes k_{(7)}\right) \\
= & u_{\gamma}\left(h_{(1)}\right) u_{\gamma}\left(k_{(1)}\right) h_{(6)} k_{(5)} R\left(S\left(h_{(5)}\right) h_{(7)} \otimes S\left(k_{(4)}\right)\right) \bar{\gamma}\left(S\left(h_{(2)}\right) \otimes h_{(10)}\right) \\
& \bar{\gamma}\left(S\left(h_{(4)}\right) h_{(8)} S\left(k_{(3)}\right) \otimes k_{(6)}\right) \bar{\gamma}\left(S\left(h_{(3)}\right) h_{(9)} \otimes S\left(k_{(2)}\right)\right),
\end{aligned}
$$


where for the last equality we used the cocycle property (4.2). Next we use the coquasitriangularity of $H$ to rewrite

$$
R\left(S\left(h_{(5)}\right) h_{(7)} \otimes S\left(k_{(4)}\right)\right) S\left(h_{(4)}\right) h_{(8)} S\left(k_{(3)}\right)=S\left(k_{(4)}\right) S\left(h_{(5)}\right) h_{(7)} R\left(S\left(h_{(4)}\right) h_{(8)} \otimes S\left(k_{(3)}\right)\right)
$$

and obtain

$$
\begin{gathered}
\mathcal{Q}(h) \dot{ }_{\gamma} \mathcal{Q}(k)=u_{\gamma}\left(h_{(1)}\right) u_{\gamma}\left(k_{(1)}\right) h_{(6)} k_{(5)} R\left(S\left(h_{(4)}\right) h_{(8)} \otimes S\left(k_{(3)}\right)\right) \bar{\gamma}\left(S\left(h_{(2)}\right) \otimes h_{(10)}\right) \\
\bar{\gamma}\left(S\left(k_{(4)}\right) S\left(h_{(5)}\right) h_{(7)} \otimes k_{(6)}\right) \bar{\gamma}\left(S\left(h_{(3)}\right) h_{(9)} \otimes S\left(k_{(2)}\right)\right) .
\end{gathered}
$$

On the other hand

$$
\begin{aligned}
& \mathcal{Q}(h k)=h_{(3)} k_{(3)} u_{\gamma}\left(h_{(1)} k_{(1)}\right) \bar{\gamma}\left(S\left(k_{(2)}\right) S\left(h_{(2)}\right) \otimes h_{(4)} k_{(4)}\right) \\
& \quad=\bar{\gamma}\left(h_{(1)} \otimes k_{(1)}\right) u_{\gamma}\left(h_{(2)}\right) u_{\gamma}\left(k_{(2)}\right) \bar{\gamma}\left(S\left(k_{(3)}\right) \otimes S\left(h_{(3)}\right)\right) h_{(5)} k_{(5)} \bar{\gamma}\left(S\left(k_{(4)}\right) S\left(h_{(4)}\right) \otimes h_{(6)} k_{(6)}\right)
\end{aligned}
$$

where we used $u_{\gamma}(h k)=\bar{\gamma}\left(h_{(1)} \otimes k_{(1)}\right) u_{\gamma}\left(h_{(2)}\right) u_{\gamma}\left(k_{(2)}\right) \bar{\gamma}\left(S\left(k_{(3)}\right) \otimes S\left(h_{(3)}\right)\right)$ that follows from the basic properties of a 2-cocycle. Then, using the explicit formula for the product $h{ }_{\underline{\gamma}} k$ given in (4.21), we have

$$
\begin{aligned}
\mathcal{Q}\left(h_{\underline{\gamma}} k\right)= & u_{\gamma}\left(h_{(1)}\right) u_{\gamma}\left(k_{(1)}\right) \bar{\gamma}\left(S\left(k_{(5)}\right) \otimes S\left(h_{(7)}\right)\right) h_{(9)} k_{(7)} \bar{\gamma}\left(S\left(k_{(6)}\right) S\left(h_{(8)}\right) \otimes h_{(10)} k_{(8)}\right) \\
& \bar{\gamma}\left(h_{(11)} \otimes k_{(9)}\right) \gamma\left(S\left(h_{(6)}\right) \otimes h_{(12)}\right) \gamma\left(S\left(k_{(4)}\right) \otimes S\left(h_{(5)}\right) h_{(13)}\right) \\
& R\left(S\left(h_{(4)}\right) h_{(14)} \otimes S\left(k_{(3)}\right)\right) \bar{\gamma}\left(S\left(h_{(3)}\right) h_{(15)} \otimes S\left(k_{(2)}\right)\right) \bar{\gamma}\left(S\left(h_{(2)}\right) \otimes h_{(16)}\right) \\
= & u_{\gamma}\left(h_{(1)}\right) u_{\gamma}\left(k_{(1)}\right) h_{(7)} k_{(6)} \bar{\gamma}\left(S\left(k_{(5)}\right) S\left(h_{(6)}\right) \otimes h_{(8)} k_{(7)}\right) \\
& \bar{\gamma}\left(h_{(9)} \otimes k_{(8)}\right) \gamma\left(S\left(k_{(4)}\right) S\left(h_{(5)}\right) \otimes h_{(10)}\right) R\left(S\left(h_{(4)}\right) h_{(11)} \otimes S\left(k_{(3)}\right)\right) \\
& \bar{\gamma}\left(S\left(h_{(3)}\right) h_{(12)} \otimes S\left(k_{(2)}\right)\right) \bar{\gamma}\left(S\left(h_{(2)}\right) \otimes h_{(13)}\right)
\end{aligned}
$$

where to obtain the last equality we used the cocycle condition (4.1) on the product $\bar{\gamma}\left(S\left(k_{(5)}\right) \otimes S\left(h_{(7)}\right)\right) \gamma\left(S\left(h_{(6)}\right) \otimes h_{(12)}\right) \gamma\left(S\left(k_{(4)}\right) \otimes S\left(h_{(5)}\right) h_{(13)}\right)$. Using once again this condition on the product $\bar{\gamma}\left(S\left(k_{(5)}\right) S\left(h_{(6)}\right) \otimes h_{(8)} k_{(7)}\right) \bar{\gamma}\left(h_{(9)} \otimes k_{(8)}\right) \gamma\left(S\left(k_{(4)}\right) S\left(h_{(5)}\right) \otimes h_{(10)}\right)$ we finally obtain

$$
\begin{gathered}
\mathcal{Q}\left(h_{\underline{\gamma}} k\right)=u_{\gamma}\left(h_{(1)}\right) u_{\gamma}\left(k_{(1)}\right) h_{(6)} k_{(5)} \bar{\gamma}\left(S\left(k_{(4)}\right) S\left(h_{(5)}\right) h_{(7)} \otimes k_{(6)}\right) R\left(S\left(h_{(4)}\right) h_{(8)} \otimes S\left(k_{(3)}\right)\right) \\
\bar{\gamma}\left(S\left(h_{(3)}\right) h_{(9)} \otimes S\left(k_{(2)}\right)\right) \bar{\gamma}\left(S\left(h_{(2)}\right) \otimes h_{(10)}\right) .
\end{gathered}
$$

Thus, $\mathcal{Q}(h) \cdot{ }_{\gamma} \mathcal{Q}(k)=\mathcal{Q}\left(h \cdot{ }_{\gamma} k\right)$.

\subsection{Twisting Hopf-Galois extensions.}

The deformation by 2-cocycles of Hopf-Galois extensions was addressed in [1] for a general Hopf algebra $H$. When $H$ is coquasitriangular one has an additional algebra structure.

Let $(H, R)$ be a coquasitriangular Hopf algebra, and $A \in \mathcal{A}_{q c}^{(H, R)}$ a quasi-commutative $H$-comodule algebra. Consider the algebra extension $B=A^{\text {coH }} \subseteq A$. Let $\gamma$ be a 2-cocycle on $H$, and consider $A_{\gamma} \in \mathcal{A}_{q c}^{\left(H_{\gamma}, R_{\gamma}\right)}$ and the corresponding algebra extension $B_{\gamma}=A_{\gamma}^{c o H_{\gamma}} \subseteq$ $A_{\gamma}$. Since the coactions $\delta^{A}: A \rightarrow A \otimes H$ and $\delta^{A_{\gamma}}: A_{\gamma} \rightarrow A_{\gamma} \otimes H_{\gamma}$ coincide, $B_{\gamma}=\bar{B}$ as $\mathbb{K}$-modules; they also coincide as algebras since $B$ carries a trivial $H$-coaction so that $m_{B_{\gamma}}=m_{B}($ see (4.9) $)$. From Theorem 2.29 both canonical maps

$$
\chi: A \otimes_{B} A \rightarrow A \otimes \underline{H} \quad \text { and } \quad \chi_{\gamma}: A_{\gamma} \otimes_{B}^{\gamma} A_{\gamma} \rightarrow A_{\gamma} \otimes^{\gamma} \underline{H_{\gamma}}
$$

are comodule algebra maps. In the context of coquasitriangular Hopf algebras and quasicommutative comodule algebras, Theorem 3.6 of [1] can be sharpened: 
Theorem 4.10. Let $(H, R)$ be a coquasitriangular Hopf algebra, and let $\gamma$ be a 2-cocycle on $H$. Let $A \in \mathcal{A}_{q c}^{(H, R)}$ with $B=A^{c o H} \subseteq A$ and twist deformation $B=A_{\gamma}^{c o H_{\gamma}} \subseteq A_{\gamma} \in$ $\mathcal{A}_{q c}^{\left(H_{\gamma}, R_{\gamma}\right)}$. Then the following diagram of morphisms in $\mathcal{A}^{H_{\gamma}}$

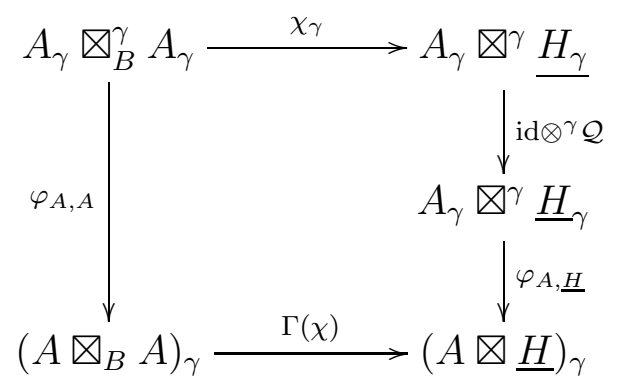

is commutative. If $H$ is cotriangular the diagram consists of morphisms in $\mathcal{A}_{q c}^{\left(H_{\gamma}, R_{\gamma}\right)}$.

Proof. In [1, Thm.3.6] the commutativity of the diagram was shown for morphisms in ${ }_{A_{\gamma}} \mathcal{M}_{A_{\gamma}}{ }^{H_{\gamma}}$, and hence in $\mathcal{M}^{H_{\gamma}}$. Since all maps in the diagram have been shown to be algebra maps (see Theorem 2.29, Proposition 4.5 and Theorem 4.9), the diagram is indeed in $\mathcal{A}^{H_{\gamma}}$. When $H$ is cotriangular, so is $H_{\gamma}$; then $\underline{H} \in \mathcal{A}_{q c}^{H}$ and also $\underline{H}_{\gamma} \simeq \underline{H}_{\gamma} \in \mathcal{A}_{q c}^{\left(H_{\gamma}, R_{\gamma}\right)}$ (from Example 2.14). Thus, due to Corollary 4.6, the diagram is in $\mathcal{A}_{q c}^{\left(H_{\gamma}, \overline{\left.R_{\gamma}\right)}\right.}$.

We remark that since all vertical arrows in the diagram (4.23) are isomorphisms, the commutativity of (4.23) implies that the extension $B \subseteq A_{\gamma}$ is an $H_{\gamma}$-Hopf-Galois extension if and only if the starting extension $B \subseteq A$ is such for the Hopf algebra $H$ (see Corollary 3.7 in [1]).

\subsection{Twisting gauge groups.}

In the hypothesis of Theorem 4.10, let $B=A^{c o H} \subseteq A$ be Hopf-Galois. The gauge group

$$
\mathcal{G}_{A_{\gamma}}:=\operatorname{Hom}_{\mathcal{A}^{H_{\gamma}}}\left(\underline{H_{\gamma}}, A_{\gamma}\right)
$$

of the Hopf-Galois extension $B=A_{\gamma}^{c o H_{\gamma}} \subseteq A_{\gamma} \in \mathcal{A}_{q c}^{\left(H_{\gamma}, R_{\gamma}\right)}$ is isomorphic to the gauge group $\mathcal{G}_{A}=\operatorname{Hom}_{\mathcal{A}^{H}}(\underline{H}, A)$ of the initial one:

Proposition 4.11. Let $(H, R)$ be a coquasitriangular Hopf algebra, $A \in \mathcal{A}_{q c}^{(H, R)}$ and let $\gamma$ be a 2-cocycle on $H$. The isomorphism $\Gamma: \operatorname{Hom}_{\mathcal{A}^{H}}(\underline{H}, A) \rightarrow \operatorname{Hom}_{\mathcal{A}^{H_{\gamma}}}\left(\underline{H}_{\gamma}, A_{\gamma}\right)$, induced by the functor $\Gamma: \mathcal{A}^{H} \rightarrow \mathcal{A}^{H_{\gamma}}$ in equation (4.8), when composed with the pullback $\mathcal{Q}^{*}: \operatorname{Hom}_{\mathcal{A}^{H_{\gamma}}}\left(\underline{H}_{\gamma}, A_{\gamma}\right) \rightarrow \operatorname{Hom}_{\mathcal{A}^{H_{\gamma}}}\left(\underline{H_{\gamma}}, A_{\gamma}\right)$ of the map $\mathcal{Q}: \underline{H}_{\gamma} \longrightarrow \underline{H}_{\gamma}$ in (4.13), gives the group isomorphism

$$
\Gamma_{\mathcal{Q}}:=\mathcal{Q}^{*} \circ \Gamma: \operatorname{Hom}_{\mathcal{A}^{H}}(\underline{H}, A) \stackrel{\simeq}{\longrightarrow} \operatorname{Hom}_{\mathcal{A}^{H \gamma}}\left(\underline{H_{\gamma}}, A_{\gamma}\right) .
$$

Explicitly, for all $h \in H_{\gamma}$, one has:

$$
\Gamma_{\mathcal{Q}}(\mathbf{f}): h \mapsto \mathbf{f}(\mathcal{Q}(h))=\mathrm{f}\left(h_{(3)}\right) u_{\gamma}\left(h_{(1)}\right) \bar{\gamma}\left(S\left(h_{(2)}\right) \otimes h_{(4)}\right) .
$$

Proof. The map $\Gamma_{\mathcal{Q}}$ is invertible since it is composition of invertible maps:

$$
\operatorname{Hom}_{\mathcal{A}^{H}}(\underline{H}, A) \stackrel{\Gamma}{\longrightarrow} \operatorname{Hom}_{\mathcal{A}^{H \gamma}}\left(\underline{H}_{\gamma}, A_{\gamma}\right) \stackrel{\mathcal{Q}^{*}}{\longrightarrow} \operatorname{Hom}_{\mathcal{A}^{H_{\gamma}}}\left(\underline{H_{\gamma}}, A_{\gamma}\right) \text {. }
$$

We are only left to verify that $\Gamma_{\mathcal{Q}}$ preserves the group product, that is that

$$
\Gamma_{\mathcal{Q}}(\mathrm{f} * \mathrm{~g})=\Gamma_{\mathcal{Q}}(\mathrm{f}) *_{\gamma} \Gamma_{\mathcal{Q}}(\mathrm{g})
$$

for each $\mathrm{f}, \mathrm{g}: \underline{H} \rightarrow A$, where $*$ denotes the convolution product in $\operatorname{Hom}_{\mathcal{A}^{H}}(\underline{H}, A)$ and $*_{\gamma}$ denotes the convolution product in $\operatorname{Hom}_{\mathcal{A}^{H_{\gamma}}}\left(\underline{H_{\gamma}}, A_{\gamma}\right)$. 
On the one hand, for each $h \in \underline{H_{\gamma}}$,

$$
\Gamma_{\mathcal{Q}}(\mathrm{f} * \mathrm{~g})(h)=\Gamma(\mathbf{f} * \mathrm{~g})(\mathcal{Q}(h))=(\mathrm{f} * \mathrm{~g})(\mathcal{Q}(h))=\mathrm{f}\left(\mathcal{Q}(h)_{(1)}\right) \mathrm{g}\left(\mathcal{Q}(h)_{(2)}\right),
$$

with the Sweedler notation $\Delta_{\underline{H}}(h)=\Delta_{H}(h)=h_{(1)} \otimes h_{(2)}$. By considering definition (4.9), in order to express the product in $A$ in terms of that in $A_{\gamma}$ we further have

$$
\begin{aligned}
\Gamma_{\mathcal{Q}}(\mathrm{f} * \mathrm{~g})(h) & =\left(\mathrm{f}\left(\mathcal{Q}\left(h_{(1)}\right)\right)\right)_{(0)} \bullet_{\gamma}\left(\mathrm{g}\left(\mathcal{Q}\left(h_{(2)}\right)\right)\right)_{(0)} \gamma\left(\left(\mathrm{f}\left(\mathcal{Q}\left(h_{(1)}\right)\right)\right)_{(1)} \otimes\left(\mathrm{g}\left(\mathcal{Q}\left(h_{(2)}\right)\right)\right)_{(1)}\right) \\
& =\mathrm{f}\left(\mathcal{Q}\left(h_{(1)}\right)_{(0)}\right) \bullet_{\gamma} \mathrm{g}\left(\mathcal{Q}\left(h_{(2)}\right)_{(0)}\right) \gamma\left(\mathcal{Q}\left(h_{(1)}\right)_{(1)} \otimes \mathcal{Q}\left(h_{(2)}\right)_{(1)}\right),
\end{aligned}
$$

where we used that $\mathrm{f}, \mathrm{g}$ are $H$-comodule maps. Recalling the definition (4.12) of the twisted coproduct $\Delta_{\underline{H}_{\gamma}}$ in $\underline{H}_{\gamma}$, the above expression simplifies to

$$
\Gamma_{\mathcal{Q}}(\mathrm{f} * \mathrm{~g})(h)=\mathrm{f}\left(\mathcal{Q}(h)_{[1]}\right) \bullet_{\gamma} \mathrm{g}\left(\mathcal{Q}(h)_{[2]}\right)
$$

with the Sweedler notation $\Delta_{\underline{H}_{\gamma}}(h)=h_{[1]} \otimes h_{[2]}$ for the components of the coproduct $\Delta_{\underline{H}_{\gamma}}$.

On the other hand

$$
\left(\Gamma_{\mathcal{Q}}(\mathrm{f}) *_{\gamma} \Gamma_{\mathcal{Q}}(\mathrm{g})\right)(h)=\mathrm{f}\left(\mathcal{Q}\left(h_{(1)}\right)\right) \bullet_{\gamma} \mathrm{g}\left(\mathcal{Q}\left(h_{(2)}\right)\right)
$$

with the Sweedler notation for the coproduct in $H_{\gamma}$ which equals that in $H$. The identity $\Gamma_{\mathcal{Q}}(\mathrm{f} * \mathrm{~g})=\Gamma_{\mathcal{Q}}(\mathrm{f}) *_{\gamma} \Gamma_{\mathcal{Q}}(\mathrm{g})$ then follows recalling that $\mathcal{Q}$ is a coalgebra map.

It is instructive to recover this isomorphism considering the gauge groups of vertical automorphisms (cf. Proposition 3.6). The gauge groups $\operatorname{Aut}_{B}(A)=\operatorname{Hom}_{B} \mathcal{A}^{H}(A, A)$ and $\operatorname{Aut}_{B}\left(A_{\gamma}\right)=\operatorname{Hom}_{B} \mathcal{A}^{H_{\gamma}}\left(A_{\gamma}, A_{\gamma}\right)$ are isomorphic via the functor $\Gamma$ :

Proposition 4.12. The restriction of the functor $\Gamma: \mathcal{A}^{H} \rightarrow \mathcal{A}^{H_{\gamma}}$ in (4.8) to ${ }_{B} \mathcal{A}^{H}$ gives a group isomorphism

$$
\Gamma: \operatorname{Aut}_{B}(A) \stackrel{\simeq}{\longrightarrow} \operatorname{Aut}_{B}\left(A_{\gamma}\right), \quad \mathrm{F}: A \rightarrow A \longmapsto \mathrm{F}_{\gamma}:=\Gamma(\mathrm{F}): A_{\gamma} \rightarrow A_{\gamma} .
$$

Proof. Since the functor $\Gamma$ is the identity on morphisms, the linear maps $\mathrm{F}$ and $\mathrm{F}_{\gamma}$ coincide. Clearly, $\Gamma$ preserves the group law which is given by the composition of maps.

The group isomorphisms in Propositions 4.11 and 4.12 above are related via the isomorphism of Proposition 3.11:

Proposition 4.13. The group isomorphisms $\underline{\theta}_{A}$ and $\underline{\theta}_{A_{\gamma}}$ given as in Proposition 3.11 , and the isomorphisms $\Gamma_{\mathcal{Q}}$ of Proposition 4.11 and $\Gamma$ of Proposition 4.12 give the following commutative diagram

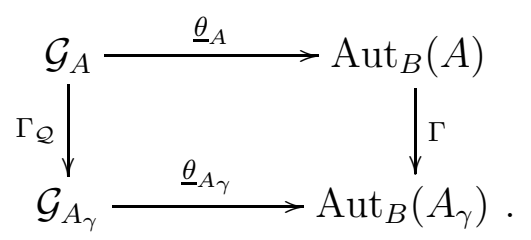

Proof. Let $\mathrm{f} \in \mathcal{G}_{A}$, we have to show that

$$
\left(\Gamma \circ \underline{\theta}_{A}\right) \mathrm{f}: A_{\gamma} \rightarrow A_{\gamma}, \quad a \mapsto a_{(0)} \mathrm{f}\left(a_{(1)}\right)
$$

coincides with

$\left(\underline{\theta}_{A_{\gamma}} \circ \Gamma_{\mathcal{Q}}\right) \mathrm{f}: A_{\gamma} \rightarrow A_{\gamma}, \quad a \mapsto a_{(0)} \bullet_{\gamma} \Gamma_{\mathcal{Q}}(\mathrm{f})\left(a_{(1)}\right)=a_{(0)} \bullet_{\gamma} \mathrm{f}\left(a_{(3)}\right) u_{\gamma}\left(a_{(1)}\right) \bar{\gamma}\left(S\left(a_{(2)}\right) \otimes a_{(4)}\right)$

Observe that

$$
a_{(0)} f\left(a_{(1)}\right)=a_{(0)} \bullet_{\gamma}\left(f\left(a_{(2)}\right)\right)_{(0)} \gamma\left(a_{(1)} \otimes\left(f\left(a_{(2)}\right)\right)_{(1)}\right)=a_{(0)} \bullet_{\gamma} f\left(a_{(3)}\right) \gamma\left(a_{(1)} \otimes S\left(a_{(2)}\right) a_{(4)}\right)
$$


where for the first equality we used (the inverse of) formula (4.9) to express the product in $A$ in terms of the product $\bullet_{\gamma}$ in $A_{\gamma}$ and for the second one $H$-equivariance of $\mathrm{f}: \underline{H} \rightarrow A$. The equality $\left(\Gamma \circ \underline{\theta}_{A}\right) \mathrm{f}=\left(\underline{\theta}_{A_{\gamma}} \circ \Gamma_{\mathcal{Q}}\right) \mathrm{f}$ then follows from the identity

$$
u_{\gamma}\left(h_{(1)}\right) \bar{\gamma}\left(S\left(h_{(2)}\right) \otimes k\right)=\gamma\left(h_{(1)} \otimes S\left(h_{(2)}\right) k\right),
$$

for all $h, k \in H$. This is shown by using the cocycle condition and the definition of $u_{\gamma}$ in (4.4), (see [1, Lem.3.2]).

Example 4.14. Noncommutative bundle over a point. Let us consider a commutative Hopf algebra $H$ with trivial $R$-form, and the trivial Hopf-Galois extension $\mathbb{K} \subseteq H$ with $H$-coaction given by the coproduct $\Delta$ (and cleaving map $j=\mathrm{id}_{H}: H \rightarrow H$ ).

Then, let $\gamma$ be a 2-cocycle on $H$. The commutative Hopf algebra $(H, R=\varepsilon \otimes \varepsilon)$ is twist deformed to the cotriangular Hopf algebra $\left(H_{\gamma}, R_{\gamma}=\gamma_{21} * \bar{\gamma}\right)$. The total space algebra $(A=H, \cdot, \Delta) \in \mathcal{A}_{q c}^{(H, R)}$ is deformed as an $H$-comodule algebra to $\left(A_{\gamma}=H_{\bullet_{\gamma}}, \bullet_{\gamma}, \Delta\right) \in$ $\mathcal{A}_{q c}^{\left(H_{\gamma}, R_{\gamma}\right)}$, and we obtain the Hopf-Galois extension $\mathbb{K} \subseteq H_{\bullet_{\gamma}} \in \mathcal{A}_{q c}^{\left(H_{\gamma}, R_{\gamma}\right)}$. Notice that this is a cleft extension with cleaving map $j=\mathrm{id}_{H}: H_{\gamma} \rightarrow H_{\bullet}$, but in general needs not be a trivial extension because $H_{\gamma}$ and $H_{\bullet_{\gamma}}$ are in general not isomorphic as $H_{\gamma}$-comodule algebras (see next examples). The gauge group of this noncommutative cleft extension $\mathbb{K} \subseteq H_{\bullet}$ is isomorphic to the gauge group of the trivial extension $\mathbb{K} \subseteq H$.

Example 4.15. Noncommutative torus bundle over a point. Let us consider the HopfGalois extension $\mathbb{C}=\mathcal{O}\left(\mathbb{T}_{\theta}^{n}\right)^{c o \mathcal{O}\left(\mathbb{T}^{n}\right)} \subseteq \mathcal{O}\left(\mathbb{T}_{\theta}^{n}\right)$ with total space the noncommutative torus $\mathbb{T}_{\theta}^{n}$ with generators $t_{j}, t_{j}^{*}$ satisfying $t_{i} t_{i}^{*}=t_{i}^{*} t_{i}=1, t_{j} t_{k}=e^{i \pi \theta_{j k}} t_{k} t_{j}$ and $t_{j} t_{k}^{*}=e^{i \pi \theta_{k j}} t_{k}^{*} t_{j}$, for $\theta_{j k}=-\theta_{k j} \in \mathbb{R}$, and with structure group the Hopf algebra $\mathcal{O}\left(\mathbb{T}^{n}\right)$.

As in Example 3.10, the $\mathcal{O}\left(\mathbb{T}^{n}\right)$-comodule map and the algebra map properties of a gauge transformation $\mathrm{F}: \mathcal{O}\left(\mathbb{T}_{\theta}^{n}\right) \rightarrow \mathcal{O}\left(\mathbb{T}_{\theta}^{n}\right)$ imply that the latter is determined by $t_{j} \mapsto F\left(t_{j}\right)=\lambda_{j} t_{j}$ and $t_{j}^{*} \mapsto F\left(t_{j}^{*}\right)=\lambda_{j}^{*} t_{j}^{*}$, with complex numbers of modulus one, $\left|\lambda_{j}\right|^{2}=1$. This shows that, independently from the noncommutativity of the generators, the gauge transformations are parametrized by $\lambda_{j} \in S^{1}$. Hence the gauge group is isomorphic to the $n$-dimensional torus $\mathbb{T}^{n}$, the same of the commutative Hopf-Galois extension $\mathbb{C}=\mathcal{O}\left(\mathbb{T}^{n}\right)^{c o \mathcal{O}\left(\mathbb{T}^{n}\right)} \subseteq \mathcal{O}\left(\mathbb{T}^{n}\right)$.

This result is consistent with the use of Proposition 4.12 for the Hopf-Galois extension $\mathbb{C} \subseteq \mathcal{O}\left(\mathbb{T}_{\theta}^{n}\right)$ seen as a twist deformation of $\mathbb{C} \subseteq \mathcal{O}\left(\mathbb{T}^{n}\right)$. The 2-cocycle $\gamma$ on $\mathcal{O}\left(\mathbb{T}^{n}\right)$ is determined by its value on the generators,

$$
\gamma\left(t_{j} \otimes t_{k}\right)=\exp \left(i \pi \theta_{j k}\right), \theta_{j k}=-\theta_{k j} \in \mathbb{R}
$$

and defined on the whole algebra by requiring $\gamma(x y \otimes z)=\gamma\left(x \otimes z_{(1)}\right) \gamma\left(y \otimes z_{(2)}\right)$ and $\gamma(x \otimes y z)=\gamma\left(x_{(1)} \otimes z\right) \gamma\left(x_{(2)} \otimes y\right)$, for all $x, y, z, \in \mathcal{O}\left(\mathbb{T}^{n}\right)$. Being the Hopf algebra $H=\mathcal{O}\left(\mathbb{T}^{n}\right)$ cocommutative, one now obtains $H_{\gamma}=H$.

Remark 4.16. When comparing the result of the previous example with Remark 3.7, we see that non isomorphic Hopf-Galois extensions $\mathbb{C} \subseteq \mathcal{O}\left(\mathbb{T}_{\theta}^{n}\right)$ (obtained from non cohomologous twists) have isomorphic gauge groups. This is a general feature occuring when starting with a cocommutative Hopf algebra $H$, which is hence transparent to the twist so that $H_{\gamma}=H$ (cf. equation (4.3)).

Example 4.17. Noncommutative $S O(2 n)$-bundle over a point. We specialise Example 4.14 to $H=\mathcal{O}(S O(2 n, \mathbb{R}))$, the algebra of coordinate functions on $S O(2 n, \mathbb{R})$. Let $\mathcal{O}(M(2 n, \mathbb{R}))$ be the commutative $*$-algebra over $\mathbb{C}$ with generators $a_{i j}, b_{i j}, a_{i j}^{*}, b_{i j}^{*}, i, j=$ 
$1, \ldots n$. It is a bialgebra with coproduct and counit given in matrix notation as

$$
\Delta(M)=M \dot{\otimes} M \quad, \quad \varepsilon(M)=\mathbb{I}_{2 n}, \quad \text { for } \quad M=\left(M_{I J}\right):=\left(\begin{array}{cc}
\left(a_{i j}\right) & \left(b_{i j}\right) \\
\left(b_{i j}^{*}\right) & \left(a_{i j}^{*}\right)
\end{array}\right) .
$$

Here $\dot{\otimes}$ is the combination of tensor product and matrix multiplication, $\mathbb{I}_{2 n}$ is the identity matrix and capital indices $I, J$ run from 1 to $2 n$. The Hopf coordinate algebra $S O(2 n, \mathbb{R})$ is the quotient $\mathcal{O}(S O(2 n, \mathbb{R}))=\mathcal{O}(M(2 n, \mathbb{R})) / I_{Q}$ with $I_{Q}$ the bialgebra ideal defined by

$$
I_{Q}=\left\langle M^{t} Q M-Q ; M Q M^{t}-Q ; \operatorname{det}(M)-1\right\rangle, \quad Q:=\left(\begin{array}{cc}
0 & \mathbb{I}_{n} \\
\mathbb{I}_{n} & 0
\end{array}\right)=Q^{t}=Q^{-1} .
$$

The $*$-structure in $\mathcal{O}(M(2 n, \mathbb{R}))$ is $M^{*}=Q M Q^{-1}$ so that $I_{Q}$ is a $*$-ideal. The $*$-bialgebra $\mathcal{O}(S O(2 n, \mathbb{R}))$ is a $*$-Hopf algebra with antipode $S(M):=Q M^{t} Q^{-1}$.

The algebra $A:=\mathcal{O}(S O(2 n, \mathbb{R}))$ is an $\mathcal{O}(S O(2 n, \mathbb{R}))$-comodule algebra with coaction the coproduct $\Delta$. The corresponding Hopf-Galois extension $\mathbb{C} \subseteq \mathcal{O}(S O(2 n, \mathbb{R}))$ is trivial and has gauge group

$$
\mathcal{G}_{A} \simeq(\{\alpha: \mathcal{O}(S O(2 n, \mathbb{R})) \rightarrow \mathbb{C} \text { algebra maps }\}, *),
$$

the set of characters of $\mathcal{O}(S O(2 n, \mathbb{R}))$ with group multiplication the convolution product.

Next we consider a 2-cocycle $\gamma$ on a maximal torus in $\mathcal{O}(S O(2 n, \mathbb{R}))$. Let $\mathcal{O}\left(\mathbb{T}^{n}\right)$ be the commutative $*$-Hopf algebra of functions on the $n$-torus as considered in Example 3.10. It is a quotient Hopf algebra, a "subgroup" of $\mathcal{O}(S O(2 n, \mathbb{R}))$, with projection

$$
\pi: M \mapsto \operatorname{diag}\left(T_{I}\right):=\operatorname{diag}\left(t_{1}, \ldots t_{n}, t_{1}^{*}, \ldots t_{n}^{*}\right) .
$$

The 2-cocycle $\gamma$ on $\mathcal{O}\left(\mathbb{T}^{n}\right)$ given in (4.24) lifts by pullback to a 2-cocycle on $\mathcal{O}(S O(2 n, \mathbb{R}))$, that we still denote by $\gamma$,

$$
\gamma\left(M_{I J} \otimes M_{K L}\right):=\gamma\left(\pi\left(M_{I J}\right) \otimes \pi\left(M_{K L}\right)\right)=\gamma\left(T_{I} \otimes T_{K}\right) \delta_{I J} \delta_{K L} .
$$

The twisted Hopf algebra $H_{\gamma}=\mathcal{O}(S O(2 n, \mathbb{R}))_{\gamma}$ has product (4.3):

$$
M_{I J} \cdot{ }_{\gamma} M_{K L}=\gamma\left(T_{I} \otimes T_{K}\right) M_{I J} M_{K L} \bar{\gamma}\left(T_{J} \otimes T_{L}\right) .
$$

Since $\gamma\left(T_{I} \otimes T_{K}\right)=\bar{\gamma}\left(T_{K} \otimes T_{I}\right)$, the generators in $\mathcal{O}(S O(2 n, \mathbb{R}))_{\gamma}$ obey relations

$$
M_{I J} \cdot{ }_{\gamma} M_{K L}=\left(\gamma\left(T_{I} \otimes T_{K}\right) \bar{\gamma}\left(T_{J} \otimes T_{L}\right)\right)^{2} M_{K L} \cdot{ }_{\gamma} M_{I J} .
$$

Explicitly, for $\lambda_{i j}=\exp \left(2 i \pi \theta_{i j}\right)$, these read

$$
\begin{array}{rlll}
a_{i j} \cdot{ }_{\gamma} a_{k l}=\lambda_{i k} \lambda_{l j} a_{k l} \cdot{ }_{\gamma} a_{i j} & , & a_{i j} \cdot{ }_{\gamma} b_{k l}^{*}=\lambda_{k i} \lambda_{l j} b_{k l}^{*} \cdot{ }_{\gamma} a_{i j}, \\
a_{i j} \cdot{ }_{\gamma} b_{k l}=\lambda_{i k} \lambda_{j l} b_{k l} \cdot{ }_{\gamma} a_{i j} & , & a_{i j} \cdot{ }_{\gamma} a_{k l}^{*}=\lambda_{k i} \lambda_{j l} a_{k l}^{*} \cdot{ }_{\gamma} a_{i j}, \\
b_{i j} \cdot{ }_{\gamma} b_{k l}=\lambda_{i k} \lambda_{l j} b_{k l} \cdot{ }_{\gamma} b_{i j} & , & b_{i j} \cdot{ }_{\gamma} b_{k l}^{*}=\lambda_{k i} \lambda_{j l} b_{k l}^{*} \cdot{ }_{\gamma} b_{i j},
\end{array}
$$

together with their $*$-conjugated. Moreover,

$$
M^{t} \cdot{ }_{\gamma} Q \cdot{ }_{\gamma} M=Q, M \cdot{ }_{\gamma} Q \cdot{ }_{\gamma} M^{t}=Q, \operatorname{det}_{\gamma}(M)=1
$$

with quantum determinant

$$
\operatorname{det}_{\gamma}(M)=\sum_{\sigma \in \mathcal{P}_{2 n}}(-1)^{|\sigma|}\left(\prod_{\substack{I<J \\ \sigma_{I}>\sigma_{J}}} \lambda_{\sigma_{I} \sigma_{J}}\right) M_{1 \sigma_{1} \cdot \gamma} \cdot{ } \cdot \gamma M_{2 n \sigma_{2 n}} .
$$

The twisted Hopf algebras $\mathcal{O}(S O(n, \mathbb{R}))_{\gamma}$ were studied in [27, 28, 8] (see also [1, §4.1]).

The comodule algebra $(A=\mathcal{O}(S O(2 n, \mathbb{R})), \Delta)$ is deformed to a comodule algebra $\left(A_{\gamma}=\mathcal{O}(S O(2 n, \mathbb{R}))_{\bullet_{\gamma}}, \Delta\right)$ with product (4.9). On the generators one has

$$
M_{I J} \bullet_{\gamma} M_{K L}=M_{I J} M_{K L} \bar{\gamma}\left(T_{J} \otimes T_{L}\right),
$$


and hence they have commutation relations

$$
M_{I J} \bullet_{\gamma} M_{K L}=\left(\bar{\gamma}\left(T_{J} \otimes T_{L}\right)\right)^{2} M_{K L} \bullet_{\gamma} M_{I J} .
$$

Explicitly,

$$
\begin{array}{cccc}
a_{i j} \bullet_{\gamma} a_{k l}=\lambda_{l j} a_{k l} \bullet_{\gamma} a_{i j} & , \quad a_{i j} \bullet_{\gamma} b_{k l}^{*}=\lambda_{l j} b_{k l}^{*} \bullet_{\gamma} a_{i j} \\
a_{i j} \bullet_{\gamma} b_{k l}=\lambda_{j l} b_{k l} \bullet_{\gamma} a_{i j} & , \quad a_{i j} \bullet_{\gamma} a_{k l}^{*}=\lambda_{j l} a_{k l}^{*} \bullet_{\gamma} a_{i j} \\
b_{i j} \bullet_{\gamma} b_{k l}=\lambda_{l j} b_{k l} \bullet_{\gamma} b_{i j} \quad, \quad b_{i j} \bullet_{\gamma} b_{k l}^{*}=\lambda_{j l} b_{k l}^{*} \bullet_{\gamma} b_{i j}
\end{array}
$$

with their $*$-conjugated. The $\mathcal{O}(S O(2 n, \mathbb{R}))_{\gamma}$-Hopf-Galois extension $\mathbb{C} \subseteq \mathcal{O}(S O(2 n, \mathbb{R}))$ • is cleft (but no longer trivial) and has gauge group $\mathcal{G}_{A_{\gamma}}$ isomorphic to $\mathcal{G}_{A}$ in (4.25).

Remark 4.18. We stress that the gauge group $\mathcal{G}_{A_{\gamma}}$ is not the group of characters of the braided Hopf algebra $H_{\gamma}$ associated with the Hopf algebra $H_{\gamma}=\mathcal{O}(S O(2 n, \mathbb{R}))_{\gamma}$ (see comment after Lemma [3.3). Indeed $H_{\gamma} \simeq \underline{H}_{\gamma}$ is genuine noncommutative. The generators of the algebra $\underline{H}_{\gamma}$ have product $\overline{(4.18)}$ :

$$
M_{I J} \cdot{ }_{\gamma} M_{K L}=M_{I J} M_{K L} \bar{\gamma}\left(S\left(T_{I}\right) T_{J} \otimes S\left(T_{K}\right) T_{L}\right),
$$

where we used that the product - coincides with that in $H=\mathcal{O}(S O(2 n, \mathbb{R}))$ since $R$ is trivial. By using the properties of the abelian cocycle $\gamma$ this product leads to commutation relations

$$
M_{I J} \dot{{ }_{\gamma}} M_{K L}=\left(\bar{\gamma}\left(T_{I} \otimes T_{K}\right) \gamma\left(T_{J} \otimes T_{K}\right) \gamma\left(T_{I} \otimes T_{L}\right) \bar{\gamma}\left(T_{J} \otimes T_{L}\right)\right)^{2} M_{K L} \dot{-} M_{I J}
$$

We see that in general the algebra $H_{\gamma} \simeq \underline{H}_{\gamma}$ is noncommutative, with less characters than the commutative algebra $H=\overline{\mathcal{O}(S O}(n, \mathbb{R}))$.

Example 4.19. Noncommutative principal bundles over affine varieties. For a principal $G$-bundle, $\pi: P \rightarrow P / G$, with $G$ a semisimple affine algebraic group and $P, P / G$ affine varieties, as in Example 2.2 we consider the $\mathcal{O}(G)$-Hopf-Galois extension $\mathcal{O}(P / G) \subseteq$ $\mathcal{O}(P)$. Given a 2-cocycle $\gamma$ on $\mathcal{O}(G)$, the gauge group of the twisted $\mathcal{O}(G)_{\gamma}$-Hopf-Galois extension $\mathcal{O}(P / G) \subseteq \mathcal{O}(P)_{\gamma}$, is isomorphic to the gauge group of $\pi: P \rightarrow P / G$.

\subsection{Tensoring Hopf-Galois extensions.}

The fiber product of a $G$-principal bundle $P \rightarrow M$ with a $G^{\prime}$-principal bundle $P^{\prime} \rightarrow M$ gives a $G \times G^{\prime}$-principal bundle $P \times_{M} P^{\prime} \rightarrow M$. The corresponding gauge group is the product of the initial gauge groups. In view of the next examples, we consider the analogue of this fiber product construction for Hopf-Galois extensions. Let $H$ and $K$ be Hopf algebras and consider an $H$-Hopf-Galois extension $B=A^{c o H} \subseteq A$ and a $K$-HopfGalois extension $B=A^{\prime c o K} \subseteq A^{\prime}$ of an algebra $B$. Assuming that $B$ is in the center of both $A$ and $A^{\prime}$, the balanced tensor product $A \otimes_{B} A^{\prime}$ inherits an algebra structure from the tensor product algebra $A \otimes A^{\prime}$. It is a comodule algebra for the Hopf algebra $H \otimes K$ (with usual tensor product algebra and coalgebra structures) with coaction

$$
\delta^{A \otimes_{B} A^{\prime}}: A \otimes_{B} A^{\prime} \rightarrow A \otimes_{B} A^{\prime} \otimes H \otimes K, \quad a \otimes_{B} a^{\prime} \mapsto a_{(0)} \otimes_{B} a_{(0)}^{\prime} \otimes a_{(1)} \otimes a_{(1)}^{\prime} .
$$

Then $B=\left(A \otimes_{B} A^{\prime}\right)^{c o(H \otimes K)} \subseteq A \otimes_{B} A^{\prime}$ is an $(H \otimes K)$-Hopf-Galois extension; indeed the corresponding canonical map has inverse

$$
\chi_{\mid 1 \otimes 1 \otimes H \otimes K}^{-1}: h \otimes k \mapsto h^{<1>} \otimes_{B} k^{<1>} \otimes_{B} h^{<2>} \otimes_{B} k^{<2>} \in\left(A \otimes_{B} A^{\prime}\right) \otimes_{B}\left(A \otimes_{B} A^{\prime}\right) .
$$

Here $\tau_{H}(h)=h^{<1>} \otimes_{B} h^{<2>}$ and $\tau_{K}(k)=k^{<1>} \otimes_{B} k^{<2>}$ denote the translation maps of the Hopf-Galois extensions $B=A^{c o H} \subseteq A$ and $B=A^{\prime c o K} \subseteq A^{\prime}$. 
If $(H, R)$ and $\left(K, R^{\prime}\right)$ are coquasitriangular then $\left(H \otimes K, R^{\prime \prime}\right)$ is coquasitriangular with $R^{\prime \prime}=\left(R \otimes R^{\prime}\right) \circ(\mathrm{id} \otimes$ flip $\otimes \mathrm{id})$, where flip $: K \otimes H \rightarrow H \otimes K, k \otimes h \mapsto h \otimes k$. Moreover, if $A \in \mathcal{A}_{q c}^{(H, R)}$ and $A^{\prime} \in \mathcal{A}_{q c}^{\left(K, R^{\prime}\right)}$, then $A \otimes_{B} A^{\prime} \in \mathcal{A}_{q c}^{\left(H \otimes K, R^{\prime \prime}\right)}$.

Proposition 4.20. Let $(H, R)$ and $\left(K, R^{\prime}\right)$ be coquasitriangular Hopf algebras. Let $B=$ $A^{c o H} \subseteq A \in \mathcal{A}_{q c}^{(H, R)}$ and $B=A^{\prime c o K} \subseteq A^{\prime} \in \mathcal{A}_{q c}^{\left(K, R^{\prime}\right)}$ be Hopf-Galois extensions, with the additional assumption that $A$ is flat as a right $B$-module and $A^{\prime}$ is flat as a left $B$-module. Then the gauge group of the tensor product $(H \otimes K)$-Hopf-Galois extension $B=\left(A \otimes_{B} A^{\prime}\right)^{c o(H \otimes K)} \subseteq A \otimes_{B} A^{\prime}$ is isomorphic to the direct product of the gauge groups of the Hopf-Galois extensions $B=A^{c o H} \subseteq A$ and $B=A^{\prime c o K} \subseteq A^{\prime}$ :

$$
\mathcal{G}_{A \otimes_{B} A^{\prime}} \simeq \mathcal{G}_{A} \times \mathcal{G}_{A^{\prime}}
$$

Proof. We consider gauge transformations as vertical automorphisms and show there is a group isomorphism

$$
\operatorname{Aut}_{B}\left(A \otimes_{B} A^{\prime}\right) \simeq \operatorname{Aut}_{B}(A) \times \operatorname{Aut}_{B}\left(A^{\prime}\right)
$$

implemented by the map

$$
\mathrm{F} \mapsto\left\{\begin{array}{l}
\mathrm{F}_{\mathrm{F}}: a \mapsto \mathrm{F}_{\mathrm{F}}(a):=\mathrm{F}\left(a \otimes_{B} 1_{A^{\prime}}\right) \\
\mathrm{F}_{\mathrm{F}}^{\prime}: a^{\prime} \mapsto \mathrm{F}_{\mathrm{F}}^{\prime}\left(a^{\prime}\right):=\mathrm{F}\left(1_{A} \otimes_{B} a^{\prime}\right)
\end{array}\right.
$$

In order to show that the image of the algebra map $\mathrm{F}_{\mathrm{F}}$ is in $A \simeq A \otimes_{B} B$ we first observe that the short exact sequence defining $A^{\prime c o K}$,

$$
0 \longrightarrow B=A^{\prime c o K} \stackrel{i}{\rightarrow} A^{\prime} \stackrel{\delta^{A^{\prime}}-\mathrm{id}_{A^{\prime}} \otimes \eta_{K}}{\longrightarrow} \operatorname{Im}\left(\delta^{A^{\prime}}-\mathrm{id}_{A^{\prime}} \otimes \eta_{K}\right) \subset A^{\prime} \otimes K \longrightarrow 0,
$$

and $B$-flatness of $A$ imply the exactness of the sequence

$$
\begin{aligned}
0 \longrightarrow A \simeq A \otimes_{B} B \stackrel{\operatorname{id}_{A} \otimes_{B} i}{\longrightarrow} & A \otimes_{B} A^{\prime} \stackrel{\operatorname{id}_{A} \otimes_{B}\left(\delta^{A^{\prime}}-\operatorname{id}_{A^{\prime}} \otimes \eta_{K}\right)}{\longrightarrow} \\
& \rightarrow \operatorname{Im}\left(\mathrm{id}_{A} \otimes_{B}\left(\delta^{A^{\prime}}-\operatorname{id}_{A^{\prime}} \otimes \eta_{K}\right)\right) \subset A \otimes_{B} A^{\prime} \otimes K \longrightarrow 0 .
\end{aligned}
$$

Then $\mathrm{F}\left(a \otimes_{B} 1_{A^{\prime}}\right) \in A \otimes_{B} B \simeq A$ follows by showing that $\mathrm{F}\left(a \otimes_{B} 1_{A^{\prime}}\right)$ is in the kernel of $\operatorname{id}_{A} \otimes_{B}\left(\delta^{A^{\prime}}-\operatorname{id}_{A^{\prime}} \otimes \eta_{K}\right)$, an easy consequence of the $(H \otimes K)$-equivariance of $\mathrm{F}$ and the identity $\left(\operatorname{id}_{A \otimes_{B} A^{\prime}} \otimes \varepsilon_{H} \otimes \mathrm{id}_{K}\right) \delta^{A \otimes_{B} A^{\prime}}=\mathrm{id}_{A} \otimes_{B} \delta^{A^{\prime}}$. Moreover, $H$-equivariance of $\mathrm{F}_{\mathrm{F}}$ follows from the identity $\left(\mathrm{id}_{A \otimes_{B} A^{\prime}} \otimes \mathrm{id}_{H} \otimes \varepsilon_{K}\right) \delta^{A \otimes_{B} A^{\prime}}=\left(\mathrm{id}_{A} \otimes_{B}\right.$ flip $) \circ\left(\delta^{A} \otimes_{B} \mathrm{id}_{A^{\prime}}\right)$.

Similarly one finds that the algebra map $\mathrm{F}_{\mathrm{F}}^{\prime}$ is a $K$-equivariant map $A^{\prime} \rightarrow B \otimes_{B} A^{\prime} \simeq A^{\prime}$.

The map $\mathrm{F} \mapsto\left(\mathrm{F}_{\mathrm{F}}, \mathrm{F}_{\mathrm{F}}^{\prime}\right)$ is an isomorphism with inverse $\left(\mathrm{F}, \mathrm{F}^{\prime}\right) \mapsto \mathrm{F}_{\left(\mathrm{F}, \mathrm{F}^{\prime}\right)}:=\mathrm{F} \otimes_{B} \mathrm{~F}^{\prime}$. This is a left inverse: $\mathrm{F} \rightarrow\left(\mathrm{F}_{\mathrm{F}}, \mathrm{F}_{\mathrm{F}}^{\prime}\right) \rightarrow \mathrm{F}_{\mathrm{F}} \otimes_{B} \mathrm{~F}_{\mathrm{F}}^{\prime}=\mathrm{F}$ because $\mathrm{F}_{\mathrm{F}}(a) \otimes_{B} \mathrm{~F}_{\mathrm{F}}^{\prime}\left(a^{\prime}\right)=$ $\left(\mathrm{F}_{\mathrm{F}}(a) \otimes_{B} 1\right)\left(1 \otimes_{B} \mathrm{~F}_{\mathrm{F}}^{\prime}\left(a^{\prime}\right)\right)=\mathrm{F}\left(a \otimes_{B} 1_{A^{\prime}}\right) \mathrm{F}\left(1_{A} \otimes_{B} a^{\prime}\right)=\mathrm{F}\left(a \otimes_{B} a^{\prime}\right)$. It is easily seen to be a right inverse as well. $\left(\mathrm{F}, \mathrm{F}^{\prime}\right) \mapsto \mathrm{F} \otimes_{B} \mathrm{~F}^{\prime} \mapsto\left(\mathrm{F}_{\mathrm{F} \otimes_{B} \mathrm{~F}^{\prime}}, \mathrm{F}_{\mathrm{F} \otimes_{B} \mathrm{~F}^{\prime}}\right)=\left(\mathrm{F}, \mathrm{F}^{\prime}\right)$.

Example 4.21. Let $H=\mathcal{O}(S U(2))$ be the Hopf coordinate algebra of the affine algebraic group $S U(2)$ and let $\mathcal{O}\left(S^{n}\right)$ denote the coordinate algebra of the classical sphere $S^{n}$. It is well-known that $A=\mathcal{O}\left(S^{7}\right)$ is a Hopf-Galois extension of $B=\mathcal{O}\left(S^{4}\right)$ for the coaction of $H$ dual to the classical principal action of $S U(2)$ on $S^{7}$ defining the $S U(2)$ Hopf bundle over $S^{4}$. The module $A=\mathcal{O}\left(S^{7}\right)$ is flat over $B=\mathcal{O}\left(S^{4}\right)$ (it is actually faithfully flat). Let $K=\mathcal{O}(U(1))$ be the Hopf algebra of coordinate functions on $U(1)$ and consider the trivial Hopf-Galois extension $B \subseteq B \otimes K$. Again $B \otimes K$ is flat as a $B$-module. In accordance with the theory above, we consider the Hopf-Galois extension for the Hopf algebra $\mathcal{O}(S U(2)) \otimes \mathcal{O}(U(1)) \simeq \mathcal{O}(S U(2) \times U(1))$ with comodule algebra $\mathcal{O}\left(S^{7}\right) \otimes_{B} B \otimes \mathcal{O}\left(S^{1}\right) \simeq \mathcal{O}\left(S^{7}\right) \otimes \mathcal{O}\left(S^{1}\right) \simeq \mathcal{O}\left(S^{7} \times S^{1}\right)$ (with obvious coaction of $\mathcal{O}(U(1))$ on $\left.\mathcal{O}\left(S^{1}\right)\right)$ and subalgebra of coinvariant elements again $B=\mathcal{O}\left(S^{4}\right)$. 
By Proposition 4.20, the commutative Hopf Galois extension $\mathcal{O}\left(S^{4}\right) \subseteq \mathcal{O}\left(S^{7}\right) \otimes \mathcal{O}\left(S^{1}\right)$ has gauge group $\mathcal{G}_{\mathcal{O}\left(S^{7}\right)} \times \mathcal{G}_{\mathcal{O}\left(S^{4}\right) \otimes \mathcal{O}(U(1))}$. Here $\mathcal{G}_{\mathcal{O}\left(S^{7}\right)}$ is the gauge group of the $S U(2)$ principal bundle $S^{7} \rightarrow S^{4}$, while $\mathcal{G}_{\mathcal{O}\left(S^{4}\right) \otimes \mathcal{O}(U(1))}=\left\{\mathrm{f}: \mathcal{O}(U(1)) \rightarrow \mathcal{O}\left(S^{4}\right)\right.$ algebra maps $\}$ is the gauge group of the trivial $U(1)$-principal bundle over $S^{4}$.

We have been working with affine varieties (noncommutative polynomial algebras of coordinates given by generators and relations). Completion in the smooth category gives the gauge group of the principal $S U(2) \times U(1)$-bundle $S^{7} \times S^{1} \rightarrow S^{4}$. Being the latter the product of the gauge group of the $S U(2)$-Hopf bundle and the group of $U(1)$ valued functions on $S^{4}$, the smooth completion of the gauge group of the commutative HopfGalois extension $\mathcal{O}\left(S^{4}\right) \subseteq \mathcal{O}\left(S^{7}\right) \otimes \mathcal{O}\left(S^{1}\right)$ is this classical gauge group.

Example 4.22. We present a twisted version of the previous example. To this aim we first twist the Hopf $*$-algebra $\mathcal{O}(S U(2)) \otimes \mathcal{O}(U(1))$. The commutative Hopf $*$-algebra $\mathcal{O}(S U(2))$ of coordinate functions on $S U(2)$ is generated by the entries of the matrix $\tilde{u}=\left(\begin{array}{ll}a & b \\ c & d\end{array}\right)$, with $c=-b^{*}, d=a^{*}$ and $a a^{*}+b b^{*}=1$. The commutative Hopf $*$-algebra $\mathcal{O}(U(1))$ of coordinate functions on $U(1)$ has generators $w, w^{*}$, with $w w^{*}=w^{*} w=1$. Consider then the tensor product Hopf *-algebra $\mathcal{O}(S U(2)) \otimes \mathcal{O}(U(1))$ and the Hopf $*$ ideal $I=\langle b \otimes 1, c \otimes 1\rangle$. The quotient Hopf algebra $\mathcal{O}(S U(2)) \otimes \mathcal{O}(U(1)) / I$ is easily seen to be isomorphic to the Hopf $*$-algebra $\mathcal{O}\left(\mathbb{T}^{2}\right)$ of functions on the 2-torus:

$$
\mathcal{O}(S U(2)) \otimes \mathcal{O}(U(1)) / I \simeq \mathcal{O}\left(\mathbb{T}^{2}\right), \quad(a \otimes 1) \mapsto t_{1}, \quad(1 \otimes w) \mapsto t_{2}
$$

The 2-cocycle $\gamma$ on $\mathcal{O}\left(\mathbb{T}^{2}\right)$ is the one given in (4.24); we choose the convention

$$
\gamma\left(t_{1} \otimes t_{2}\right)=\gamma\left(t_{2} \otimes t_{1}\right)^{-1}=\exp \left(i \pi \frac{1}{2} \theta\right) .
$$

Via the quotient map $\pi: \mathcal{O}(S U(2)) \otimes \mathcal{O}(U(1)) \rightarrow \mathcal{O}(S U(2)) \otimes \mathcal{O}(U(1)) / I \simeq \mathcal{O}\left(\mathbb{T}^{2}\right)$ it lifts to a 2-cocycle $\gamma$ on $\mathcal{O}(S U(2)) \otimes \mathcal{O}(U(1))$ defined, for all $\ell, \ell^{\prime} \in \mathcal{O}(S U(2)) \otimes \mathcal{O}(U(1))$, by (see [1, Lem.4.1])

$$
\gamma\left(\ell \otimes \ell^{\prime}\right):=\gamma\left(\pi(\ell) \otimes \pi\left(\ell^{\prime}\right)\right) .
$$

With this 2-cocycle we deform the Hopf algebra $\mathcal{O}(S U(2)) \otimes \mathcal{O}(U(1))$ to the new Hopf algebra $(\mathcal{O}(S U(2)) \otimes \mathcal{O}(U(1)))_{\gamma}$ with product given in (4.3). Working out the commutation relations, one finds this Hopf algebra to be generated by the elements

$$
a:=a \otimes 1, \quad b:=b \otimes 1, \quad w:=1 \otimes w,\left(\text { together with } a^{*}, b^{*}, w^{*}\right)
$$

where $a$ is central (and so is $a^{*}$ ), modulo the further commutation relations

$$
b \cdot{ }_{\gamma} b^{*}=b^{*} \cdot{ }_{\gamma} b, \quad w \cdot{ }_{\gamma} w^{*}=w^{*} \cdot{ }_{\gamma} w, \quad b \cdot{ }_{\gamma} w=q w \cdot{ }_{\gamma} b, \quad b \cdot{ }_{\gamma} w^{*}=\bar{q} w^{*} \cdot{ }_{\gamma} b,
$$

with $q:=\exp (2 \pi i \theta)$, and modulo the relations

$$
a \cdot{ }_{\gamma} a^{*}+b \cdot{ }_{\gamma} b^{*}=1 \quad, \quad w \cdot{ }_{\gamma} w^{*}=1 .
$$

Counit and coproduct are undeformed and given by $\varepsilon(a)=1, \varepsilon(b)=0, \varepsilon(w)=1$ and

$$
\Delta(a)=a \otimes a-b \otimes b^{*}, \quad \Delta(b)=a \otimes b+b \otimes a^{*}, \quad \Delta(w)=w \otimes w .
$$

The antipode, $S_{\gamma}:=u_{\gamma} * S * \bar{u}_{\gamma}$, where $u_{\gamma}$ and $\bar{u}_{\gamma}$ are in (4.4), on the generators reads

$$
S_{\gamma}(a)=S(a)=a^{*}, \quad S_{\gamma}(b)=S(b)=-b, \quad S_{\gamma}(w)=S(w)=w^{*} .
$$

We next deform the Hopf-Galois extension of Example 4.21 to a new Hopf-Galois extension over $B=\mathcal{O}\left(S^{4}\right)$ with Hopf algebra just $(\mathcal{O}(S U(2)) \otimes \mathcal{O}(U(1)))_{\gamma}$. The total space is the noncommutative algebra $\left(\mathcal{O}\left(S^{7}\right) \otimes \mathcal{O}\left(S^{1}\right)\right)_{\gamma}$, deformation of the algebra $\mathcal{O}\left(S^{7}\right) \otimes \mathcal{O}\left(S^{1}\right)$ as in (4.9). Explicitly, since $\mathcal{O}\left(S^{7}\right) \otimes \mathcal{O}\left(S^{1}\right)$ is the commutative $*$-algebra 
generated by $z_{j}, z_{j}^{*}$, for $j=1,2,3,4$, and $w, w^{*}$, then $\left(\mathcal{O}\left(S^{7}\right) \otimes \mathcal{O}\left(S^{1}\right)\right)_{\gamma}$ is the noncommutative $*$-algebra generated by $z_{j}, z_{j}^{*}$, and $w, w^{*}$, modulo the commutation relations

$$
z_{j} \bullet_{\gamma} z_{k}=z_{k} \bullet_{\gamma} z_{j} ; \quad z_{j} \bullet_{\gamma} w=\exp (-i \pi \theta) w \bullet_{\gamma} z_{j} ; \quad z_{j}^{*} \bullet_{\gamma} w=\exp (i \pi \theta) w \bullet_{\gamma} z_{j}^{*},
$$

for each $j, k=1, \ldots, 4$ (and their $*$-conjugates), and the relations

$$
\sum_{j=1}^{4} z_{j}^{*} \bullet_{\gamma} z_{j}=1 \quad ; \quad w^{*} \bullet_{\gamma} w=1 .
$$

Thus the subalgebras $\mathcal{O}\left(S^{7}\right)$ and $\mathcal{O}\left(S^{1}\right)$ remain commutative, and the noncommutativity can therefore be ascribed just to the tensor product in $\left(\mathcal{O}\left(S^{7}\right) \otimes \mathcal{O}\left(S^{1}\right)\right)_{\gamma} \simeq \mathcal{O}\left(S^{7} \times S^{1}\right)_{\gamma}$, suggesting the notation $\mathcal{O}\left(S^{7}\right) \otimes_{\gamma} \mathcal{O}\left(S^{1}\right)$. Indeed this algebra is of the same type as those obtained in [15] as noncommutative products of spheres, there denoted $\mathcal{O}\left(S^{7} \times_{\gamma} S^{1}\right)$.

By Proposition 4.11 we conclude that the gauge group of the Hopf-Galois extension $\mathcal{O}\left(S^{4}\right) \subseteq\left(\mathcal{O}\left(S^{7}\right) \otimes \mathcal{O}\left(S^{1}\right)\right)_{\gamma}$ is isomorphic to that of the commutative Hopf-Galois extension $\mathcal{O}\left(S^{4}\right) \subseteq \mathcal{O}\left(S^{7}\right) \otimes \mathcal{O}\left(S^{1}\right)$ described in the previous example (that is, the gauge group of the $S U(2)$-Hopf bundle times the group of $U(1)$ valued functions on $S^{4}$ ).

Example 4.23. Reduction of the "structure group". Given any odd dimensional sphere $S^{2 n-1}$, the cartesian product $S^{2 n-1} \times S^{1}$ carries a diagonal action of $\mathbb{Z}_{2}$, with the non trivial generator of the latter sending a point on a sphere to its antipodal point. The quotient $\left(S^{2 n-1} \times S^{1}\right) / \mathbb{Z}_{2}$ is a copy of $S^{2 n-1} \times S^{1}:$ if $z_{j}, z_{j}^{*}, j=1, \ldots, n$, are coordinates on $S^{2 n-1}$ with $\sum_{j=1}^{n} z_{j}^{*} z_{j}=1$ and $w$ is the coordinate of $S^{1}$ with $w^{*} w=1$, coordinates for the quotient are given by $x_{j}=z_{j} w$ and $y=\left(w^{*}\right)^{2}$.

On the other hand, with the group structures of $S^{3}=S U(2)$ and $S^{1}=U(1)$, the quotient group $(S U(2) \times U(1)) / \mathbb{Z}_{2}$ is isomorphic to the group $U(2)$. Consider the principal $S U(2) \times S^{1}$ bundle $S^{7} \times S^{1} \rightarrow S^{4}$. The subgroup $\mathbb{Z}_{2}$ of $S U(2) \times U(1)$ acts on $S^{7} \times S^{1}$ as above by flipping antipodal points; then the quotient leads to an $(S U(2) \times U(1)) / \mathbb{Z}_{2} \simeq$ $U(2)$ bundle with total space $\left(S^{7} \times S^{1}\right) / \mathbb{Z}_{2} \simeq S^{7} \times S^{1}$ and base space still $S^{4}$.

We next present an Hopf-Galois description of this construction that also applies to the noncommutative Hopf-Galois extension $\mathcal{O}\left(S^{4}\right) \subseteq\left(\mathcal{O}\left(S^{7}\right) \otimes \mathcal{O}\left(S^{1}\right)\right)_{\gamma}$ and leads to an $\mathcal{O}\left(U_{q}(2)\right)$-Hopf-Galois extension, with corresponding gauge group.

Example 4.24. Twisting Example 4.23. The *-algebras $(\mathcal{O}(S U(2)) \otimes \mathcal{O}(U(1)))_{\gamma}$ and $\left(\mathcal{O}\left(S^{7}\right) \otimes \mathcal{O}\left(S^{1}\right)\right)_{\gamma}$ are both $\mathbb{Z}_{2}$-graded with the generators that are odd, while the commutation relations, determinant and radius relations (4.29)-(4.30) and (4.33)-(4.34) are even; the $*$-involutions are grade preserving.

Firstly, consider the $\mathbb{Z}_{2}$-invariant $*$-algebra $\left(\mathcal{O}\left(S^{7}\right) \otimes \mathcal{O}\left(S^{1}\right)\right)_{\gamma}^{\mathbb{Z}_{2}} \subseteq\left(\mathcal{O}\left(S^{7}\right) \otimes \mathcal{O}\left(S^{1}\right)\right)_{\gamma}$. It is easy seen to be generated by the elements $x_{j}:=z_{j} \bullet_{\gamma} w$ and $y:=w^{*} \bullet_{\gamma} w^{*}$, and their *-conjugates $x_{j}^{*}$ and $y^{*}$, modulo commutation relations induced by (4.33):

$$
x_{j} \bullet_{\gamma} x_{k}=x_{k} \bullet_{\gamma} x_{j}, \quad x_{j} \bullet_{\gamma} y=q y \bullet_{\gamma} x_{j}, x_{j}^{*} \bullet_{\gamma} y=q^{-1} y \bullet_{\gamma} x_{j}^{*},
$$

(with their $*$-conjugates) and radius relations $\sum_{j} x_{j}^{*} \bullet_{\gamma} x_{j}=1, y \bullet_{\gamma} y^{*}=1$ induced by (4.34). Here $q=\exp (2 \pi i \theta)$. We see that $\left(\mathcal{O}\left(S^{7}\right) \otimes \mathcal{O}\left(S^{1}\right)\right)_{\gamma}^{\mathbb{Z}_{2}}$ is again a deformation of the algebra of coordinates of the affine variety $S^{7} \times S^{1}$ as in (4.33), but with different noncommutativity parameter $q=\exp (2 \pi i \theta)$ rather than $\exp (-i \pi \theta)$.

Next, the even $*$-subalgebra $(\mathcal{O}(S U(2)) \otimes \mathcal{O}(U(1)))_{\gamma}^{\mathbb{Z}_{2}}$ is also a Hopf $*$-subalgebra of $(\mathcal{O}(S U(2)) \otimes \mathcal{O}(U(1)))_{\gamma}$ since the coproduct and the antipode are grade preserving and 
hence restrict to the even subalgebra. This Hopf $*$-subalgebra is easily seen to be the algebra generated by the elements

$$
\alpha:=a \cdot{ }_{\gamma} w, \quad \beta:=b \cdot{ }_{\gamma} w, \quad D:=w \cdot{ }_{\gamma} w,
$$

and their $*$-conjugated elements $\alpha^{*}=w^{*} \cdot{ }_{\gamma} a^{*}, \beta^{*}=w^{*} \cdot{ }_{\gamma} b^{*}, D^{*}=w^{*} \cdot{ }_{\gamma} w^{*}$, modulo the commutation relations induced by (4.29), which are worked out to be

$$
\begin{aligned}
& \alpha \cdot{ }_{\gamma} \beta=q^{-1} \beta \cdot{ }_{\gamma} \alpha, \quad \alpha \cdot{ }_{\gamma} \beta^{*}=q \beta^{*} \cdot{ }_{\gamma} \alpha, \\
& D \cdot{ }_{\gamma} \alpha=\alpha \cdot{ }_{\gamma} D, \quad D \cdot{ }_{\gamma} \alpha^{*}=\alpha^{*} \cdot{ }_{\gamma} D, \\
& D \cdot{ }_{\gamma} \beta=q^{-2} \beta \cdot{ }_{\gamma} D, \quad D \cdot{ }_{\gamma} \beta^{*}=q^{2} \beta^{*} \cdot{ }_{\gamma} D
\end{aligned}
$$

(together with their $*$-conjugates) and the relations

$$
\alpha \cdot{ }_{\gamma} \alpha^{*}+\beta \cdot{ }_{\gamma} \beta^{*}=1, \quad D \cdot{ }_{\gamma} D^{*}=1 .
$$

When restricting the coproduct in (4.31) to the subalgebra one obtains:

$$
\Delta(\alpha)=\alpha \otimes \alpha-q \beta \otimes\left(\beta^{*} \cdot{ }_{\gamma} D\right), \quad \Delta(\beta)=\alpha \otimes \beta+\beta \otimes\left(\alpha^{*} \cdot{ }_{\gamma} D\right), \quad \Delta(D)=D \otimes D .
$$

Similarly, restricting the counit one has $\varepsilon(\alpha)=1, \varepsilon(\beta)=0, \varepsilon(D)=1$, and finally for the antipode (4.32) one finds

$$
S(\alpha)=\alpha^{*}, \quad S(\beta)=-q\left(\beta \cdot_{\gamma} D^{*}\right), \quad S(D)=D^{*} .
$$

Paralleling the classical result for the group $(S U(2) \times U(1)) / \mathbb{Z}_{2} \simeq U(2)$, the Hopf $*$ algebra $(\mathcal{O}(S U(2)) \otimes \mathcal{O}(U(1)))_{\gamma}^{\mathbb{Z}_{2}}$ (with the structures in (4.38) and (4.39) $)$ is isomorphic to the cotriangular quantum group $\mathcal{O}\left(U_{q}(2)\right)$ (the multiparametric quantum group $U_{q, r}(2)$ with $r=1$, see e.g. [28, 27]). We recall that $\mathcal{O}\left(U_{q}(2)\right)$ is generated by the matrix entries, $u:=\left(\begin{array}{ll}\alpha & \beta \\ \gamma & \delta\end{array}\right)$, and by the inverse $D^{-1}$ of the quantum determinant $D=\alpha \delta-q^{-1} \beta \gamma$. These generators satisfy the FRT commutation relations $\mathcal{R}_{k l}^{j i} u_{k m} u_{l n}=u_{i k} u_{j l} \mathcal{R}_{m n}^{l k}$, with matrix $\mathcal{R}=\operatorname{diag}\left(1, q^{-1}, q, 1\right)$. Explicitly, we have

$$
\begin{aligned}
& \alpha \beta=q^{-1} \beta \alpha ; \quad \alpha \gamma=q \gamma \alpha ; \quad \beta \delta=q \delta \beta ; \quad \gamma \delta=q^{-1} \delta \gamma ; \quad \beta \gamma=q^{2} \gamma \beta ; \quad \alpha \delta=\delta \alpha \\
& \alpha D^{-1}=D^{-1} \alpha ; \quad \beta D^{-1}=q^{-2} D^{-1} \beta ; \quad \gamma D^{-1}=q^{2} D^{-1} \gamma ; \quad \delta D^{-1}=D^{-1} \delta .
\end{aligned}
$$

The costructures are $\Delta(u)=u \dot{\otimes} u, \Delta\left(D^{-1}\right)=D^{-1} \otimes D^{-1}$, and $\varepsilon(u)=\mathbb{I}_{2}, \varepsilon\left(D^{-1}\right)=1$, while the antipode is

$$
S(u)=D^{-1}\left(\begin{array}{cc}
\delta & -q^{-1} \beta \\
-q \gamma & \alpha
\end{array}\right), \quad S\left(D^{-1}\right)=D .
$$

The $*$-structure defining the real form $\mathcal{O}\left(U_{q}(2)\right)$ requires the deformation parameter to be a phase, we set $q=\exp (2 \pi i \theta)$ as in (4.29). The $*$-structure is then given by

$$
\left(\begin{array}{ll}
\alpha^{*} & \beta^{*} \\
\gamma^{*} & \delta^{*}
\end{array}\right)=D^{-1}\left(\begin{array}{cc}
\delta & -q \gamma \\
-q^{-1} \beta & \alpha
\end{array}\right), \quad\left(D^{-1}\right)^{*}=D
$$

The defining relations (4.36) and (4.37) are the same as the FRT commutation relations (4.40) and the relations $D=\alpha \delta-q^{-1} \beta \gamma, D D^{-1}=1$ with $\delta=D \alpha^{*}$ and $\gamma=-q^{-1} D \beta^{*}$ as given in (4.42). The costructures and antipode too in (4.38) and (4.39) are those of $\mathcal{O}\left(U_{q}(2)\right)$, thus showing the isomorphism $(\mathcal{O}(S U(2)) \otimes \mathcal{O}(U(1)))_{\gamma}^{\mathbb{Z}_{2}} \simeq \mathcal{O}\left(U_{q}(2)\right)$ as Hopf 
*-algebras. We have obtained the quantum group $\mathcal{O}\left(U_{q}(2)\right)$ from the "quantum double cover" $(\mathcal{O}(S U(2)) \otimes \mathcal{O}(U(1)))_{\gamma}[1$ Finally, the coaction

$$
\left(\mathcal{O}\left(S^{7}\right) \otimes \mathcal{O}\left(S^{1}\right)\right)_{\gamma} \rightarrow\left(\mathcal{O}\left(S^{7}\right) \otimes \mathcal{O}\left(S^{1}\right)\right)_{\gamma} \otimes(\mathcal{O}(S U(2)) \times \mathcal{O}(U(1)))_{\gamma}
$$

is even and therefore, restricted to $\left(\mathcal{O}\left(S^{7}\right) \otimes \mathcal{O}\left(S^{1}\right)\right)_{\gamma}^{\mathbb{Z}_{2}}$, defines an $\mathcal{O}\left(U_{q}(2)\right)$-coaction

$$
\left(\mathcal{O}\left(S^{7}\right) \otimes \mathcal{O}\left(S^{1}\right)\right)_{\gamma}^{\mathbb{Z}_{2}} \rightarrow\left(\mathcal{O}\left(S^{7}\right) \otimes \mathcal{O}\left(S^{1}\right)\right)_{\gamma}^{\mathbb{Z}_{2}} \otimes \mathcal{O}\left(U_{q}(2)\right)
$$

It then follows that the subalgebra $\mathcal{O}\left(S^{4}\right) \subseteq\left(\mathcal{O}\left(S^{7}\right) \otimes \mathcal{O}\left(S^{1}\right)\right)_{\gamma}$ of $(\mathcal{O}(S U(2)) \otimes \mathcal{O}(U(1)))_{\gamma}$ coinvariants, being even, coincides with the subalgebra $\mathcal{O}\left(S^{4}\right) \subseteq\left(\mathcal{O}\left(S^{7}\right) \otimes \mathcal{O}\left(S^{1}\right)\right)_{\gamma}^{\mathbb{Z}_{2}}$ of $\mathcal{O}\left(U_{q}(2)\right)$-coinvariants. Furthermore, since the canonical map of the initial Hopf-Galois extension $\mathcal{O}\left(S^{4}\right) \subseteq\left(\mathcal{O}\left(S^{7}\right) \otimes \mathcal{O}\left(S^{1}\right)\right)_{\gamma}$ is even, the extension $\mathcal{O}\left(S^{4}\right) \subseteq\left(\mathcal{O}\left(S^{7}\right) \otimes \mathcal{O}\left(S^{1}\right)\right)_{\gamma}^{\mathbb{Z}_{2}}$ is Hopf-Galois as well.

We further observe that the $\mathcal{O}\left(U_{q}(2)\right)$-Hopf-Galois extension is a 2-cocycle deformation of the commutative $\mathcal{O}(U(2))$-Hopf-Galois extension: the 2-cocycle on $\mathcal{O}(U(2)) \simeq$ $(\mathcal{O}(S U(2)) \otimes \mathcal{O}(U(1)))^{\mathbb{Z}_{2}}$ is the restriction of the one on $\mathcal{O}(S U(2)) \otimes \mathcal{O}(U(1))$. The corresponding deformation of $\left(\mathcal{O}\left(S^{7}\right) \otimes \mathcal{O}\left(S^{1}\right)\right)^{\mathbb{Z}_{2}}$ via the $\mathcal{O}(U(2))$-coaction is then the deformation of $\left(\mathcal{O}\left(S^{7}\right) \otimes \mathcal{O}\left(S^{1}\right)\right)^{\mathbb{Z}_{2}} \subseteq \mathcal{O}\left(S^{7}\right) \otimes \mathcal{O}\left(S^{1}\right)$ via the $\mathcal{O}(S U(2)) \otimes \mathcal{O}(U(1))$ coaction. From Proposition 4.11 we conclude that the gauge group of this noncommutative $\mathcal{O}\left(U_{q}(2)\right)$-Hopf-Galois extension is undeformed.

\section{Appendix A. The Canonical map as a morphism of Relative Hopf modules}

As mentioned in 22.1 , for a generic Hopf algebra $H$ (that is, not necessarily coquasitriangular), the canonical map (2.1) of a Hopf-Galois extension $B \subseteq A$,

$$
\chi=(m \otimes \mathrm{id}) \circ\left(\mathrm{id} \otimes_{B} \delta^{A}\right): A \otimes_{B} A \longrightarrow A \otimes H, \quad a^{\prime} \otimes_{B} a \longmapsto a^{\prime} a_{(0)} \otimes a_{(1)},
$$

was shown in [1, §2] to be a a morphism in the category ${ }_{A} \mathcal{M}_{A}{ }^{H}$ of relative Hopf modules.

It was proved in [29, §1.1] that $\chi$ is a morphism in $\mathcal{M}_{A}{ }^{H}$ when $A \otimes_{B} A$ and $A \otimes H$ are seen as objects in $\mathcal{M}_{A}{ }^{H}$ with right $A$-module structures

$$
\left(a \otimes_{B} a^{\prime}\right) a^{\prime \prime}:=a \otimes_{B} a^{\prime} a^{\prime \prime} \quad \text { and } \quad(a \otimes h) a^{\prime}=a a_{(0)}^{\prime} \otimes h a_{(1)}^{\prime}
$$

and right $H$-coactions: for all $a, a^{\prime}, a^{\prime \prime} \in A, h \in H$,

$$
a \otimes_{B} a^{\prime} \mapsto a \otimes a_{(0)}^{\prime} \otimes a_{(1)}^{\prime} \quad \text { and } \quad a \otimes h \mapsto a \otimes h_{(1)} \otimes h_{(2)} .
$$

Moreover, $\chi$ is a morphism in ${ }_{A} \mathcal{M}^{H}$ when $A \otimes_{B} A$ and $A \otimes H$ are considered to be objects in ${ }_{A} \mathcal{M}^{H}$ with left $A$-module structures given by left multiplication on the first factors and right $H$-coactions: for all $a, a^{\prime} \in A, h \in H$,

$$
a \otimes_{B} a^{\prime} \mapsto a_{(0)} \otimes a^{\prime} \otimes a_{(1)} \quad \text { and } \quad a \otimes h \mapsto a_{(0)} \otimes h_{(2)} \otimes a_{(1)} S\left(h_{(1)}\right) .
$$

In [1, §2] both $A \otimes_{B} A$ and $A \otimes H$ were shown to be objects in ${ }_{A} \mathcal{M}_{A}{ }^{H}$, with $\chi$ a morphism in the category ${ }_{A} \mathcal{M}_{A}{ }^{H}$ of relative Hopf modules. As already recalled in $₫ 2.1$, the left $A$-module structures are the left multiplication on the first factors and the right $A$-actions as in (A.1). The tensor product $A \otimes_{B} A$ carries the right $H$-coaction in (2.2):

$$
\delta^{A \otimes_{B} A}: A \otimes_{B} A \rightarrow A \otimes_{B} A \otimes H, \quad a \otimes_{B} a^{\prime} \mapsto a_{(0)} \otimes_{B} a_{(0)}^{\prime} \otimes a_{(1)} a_{(1)}^{\prime}
$$

\footnotetext{
${ }^{1}$ The coproduct (4.38) and antipode (4.39) also show a semidirect structure of the Hopf $*$-algebra $\mathcal{O}\left(U_{q}(2)\right)$ that corresponds to the semidirect product $S U(2) \rtimes U(1) \simeq U(2) \simeq(S U(2) \times U(1)) / \mathbb{Z}_{2}$, obtained by decomposing $U(2)$ matrices as $\left(\begin{array}{cc}\alpha & \beta \\ \gamma & \delta\end{array}\right)=\left(\begin{array}{cc}\alpha & \beta \\ -D \beta^{*} & D \alpha^{*}\end{array}\right)=\left(\begin{array}{cc}1 & 0 \\ 0 & D\end{array}\right)\left(\begin{array}{cc}\alpha & \beta \\ -\beta^{*} & \alpha^{*}\end{array}\right)$.
} 
for all $a, a^{\prime} \in A$. The right $H$-coaction on $A \otimes H$ is given by (2.4): for all $a \in A, h \in H$,

$$
\delta^{A \otimes H}(a \otimes h)=a_{(0)} \otimes h_{(2)} \otimes a_{(1)} S\left(h_{(1)}\right) h_{(3)} \in A \otimes H \otimes H .
$$

These two approaches can be related: the coactions (A.4) and (A.5) can be obtained as the compositions of the coactions (A.3) and (A.2) in the sense of the following lemma.

Lemma A.1. Let $\left(V, \delta_{1}, \delta_{2}\right)$ be a relative Hopf module in $\mathcal{M}^{H, H}$, that is $V$ is a $\mathbb{K}$-module endowed with two coactions $\delta_{1}: v \mapsto v_{(0)} \otimes v_{(1)}$ and $\delta_{2}: v \mapsto v^{(0)} \otimes v^{(1)}$ of an Hopf algebra $H$ compatible in the sense that

$$
\left(\delta_{1} \otimes \mathrm{id}_{H}\right) \circ \delta_{2}=\left(\mathrm{id}_{V} \otimes \operatorname{flip}\right) \circ\left(\delta_{2} \otimes \mathrm{id}_{H}\right) \circ \delta_{1}
$$

that is for all $v \in V$

$$
\left(v^{(0)}\right)_{(0)} \otimes\left(v^{(0)}\right)_{(1)} \otimes v^{(1)}=\left(v_{(0)}\right)^{(0)} \otimes v_{(1)} \otimes\left(v_{(0)}\right)^{(1)} .
$$

Then the compositions

$$
\begin{array}{ll}
\delta_{2} \circ \delta_{1}:=\left(\mathrm{id}_{V} \otimes m_{H}\right) \circ\left(\delta_{2} \otimes \mathrm{id}_{H}\right) \circ \delta_{1}: & v \mapsto\left(v_{(0)}\right)^{(0)} \otimes\left(v_{(0)}\right)^{(1)} v_{(1)} \\
\delta_{1} \circ \delta_{2}:=\left(\mathrm{id}_{V} \otimes m_{H}\right) \circ\left(\delta_{1} \otimes \mathrm{id}_{H}\right) \circ \delta_{2}: & v \mapsto\left(v^{(0)}\right)_{(0)} \otimes\left(v^{(0)}\right)_{(1)} v^{(1)}
\end{array}
$$

define new coactions on $V$.

Proof. Notice that condition (A.7) is symmetric for the exchange $\delta_{1} \leftrightarrow \delta_{2}$ and so it is enough to prove the result for (say) $\delta:=\delta_{2} \circ \delta_{1}$. It is easy to verify that $\left(\mathrm{id}_{V} \otimes \varepsilon\right) \circ \delta=\mathrm{id}_{V}$. We have to show that $\left(\operatorname{id}_{V} \otimes \Delta\right) \circ \delta=\left(\delta \otimes \operatorname{id}_{H}\right) \circ \delta$. Let $v \in V$, then

$$
\begin{aligned}
\left(\mathrm{id}_{V} \otimes \Delta\right) \circ \delta(v) & =\left(v_{(0)}\right)^{(0)} \otimes \Delta\left(\left(v_{(0)}\right)^{(1)} v_{(1)}\right) \\
& =\left(v_{(0)}\right)^{(0)} \otimes\left(\left(v_{(0)}\right)^{(1)}\right)_{(1)} v_{(1)} \otimes\left(\left(v_{(0)}\right)^{(1)}\right)_{(2)} v_{(2)} \\
& =\left(v_{(0)}\right)^{(0)} \otimes\left(v_{(0)}\right)^{(1)} v_{(1)} \otimes\left(v_{(0)}\right)^{(2)} v_{(2)}
\end{aligned}
$$

where we used the fact that $\delta_{2}$ is a comodule map:

$$
v^{(0)} \otimes\left(v^{(1)}\right)_{(1)} \otimes\left(v^{(1)}\right)_{(2)}=\left(v^{(0)}\right)^{(0)} \otimes\left(v^{(0)}\right)^{(1)} \otimes v^{(1)}=: v^{(0)} \otimes v^{(1)} \otimes v^{(2)},
$$

for $v \in V$. On the other hand

$$
\begin{aligned}
\left(\delta \otimes \mathrm{id}_{H}\right) \circ \delta(v) & =\delta\left(\left(v_{(0)}\right)^{(0)}\right) \otimes\left(v_{(0)}\right)^{(1)} v_{(1)} \\
& =\left(\left(\left(v_{(0)}\right)^{(0)}\right)_{(0)}\right)^{(0)} \otimes\left(\left(\left(v_{(0)}\right)^{(0)}\right)_{(0)}\right)^{(1)}\left(\left(v_{(0)}\right)^{(0)}\right)_{(1)} \otimes\left(v_{(0)}\right)^{(1)} v_{(1)} \\
& =\left(\left(\left(v_{(0)}\right)^{(0)}\right)^{(0)}\right)_{(0)} \otimes\left(\left(v_{(0)}\right)^{(0)}\right)^{(1)}\left(\left(\left(v_{(0)}\right)^{(0)}\right)^{(0)}\right)_{(1)} \otimes\left(v_{(0)}\right)^{(1)} v_{(1)} \\
& =\left(\left(v_{(0)}\right)^{(0)}\right)_{(0)} \otimes\left(v_{(0)}\right)^{(1)}\left(\left(v_{(0)}\right)^{(0)}\right)_{(1)} \otimes\left(v_{(0)}\right)^{(2)} v_{(1)}
\end{aligned}
$$

where the third equality follows from the condition (A.7) on $\left(v_{(0)}\right)^{(0)}$ and the last one from the fact that $\delta_{2}$ is a comodule map. By using once again condition (A.7) on $v_{(0)}$ we obtain

$$
\begin{aligned}
\left(\delta \otimes \operatorname{id}_{H}\right) \circ \delta(v) & =\left(\left(v_{(0)}\right)_{(0)}\right)^{(0)} \otimes\left(\left(v_{(0)}\right)_{(0)}\right)^{(1)}\left(v_{(0)}\right)_{(1)} \otimes\left(v_{(0)}\right)^{(2)} v_{(1)} \\
& =\left(v_{(0)}\right)^{(0)} \otimes\left(v_{(0)}\right)^{(1)} v_{(1)} \otimes\left(v_{(0)}\right)^{(2)} v_{(2)}
\end{aligned}
$$

where the last equality uses that $\delta_{1}$ is a comodule map. By comparison with the formula obtained before for $\left(\operatorname{id}_{V} \otimes \Delta\right) \circ \delta(v)$ we can conclude that $\delta$ is a comodule map.

On the $H$ comodule $A \otimes_{B} A$, with $\delta_{1}$ the coaction in (A.2) and $\delta_{2}$ the coaction in (A.3), the condition (A.7) is satisfied and their composition $\delta_{2} \circ \delta_{1}$, defined as in (A.8), is just the coaction in (A.4). On the other hand, on the $H$ comodule $A \otimes H$ with coactions $\delta_{1}$ as in (A.2) and $\delta_{2}$ as in (A.3), condition (A.7) is satisfied as well and their composition $\delta_{2} \circ \delta_{1}$ is the tensor product coaction in (A.5). 
A.1. The translation map as a morphism of relative Hopf modules. The condition for the canonical map $\chi$ to be a morphism of relative Hopf modules can equivalently be expressed in terms of its inverse. In particular, it allows to infer the following properties of the translation map $\left.\chi^{-1}\right|_{1 \otimes \underline{H}}=\tau: H \rightarrow A \otimes_{B} A$ (cf. [3, Prop.3.6]).

The condition that $\chi^{-1}$ is an $H$-comodule map with respect to the $H$-coactions in (A.2) is $\chi^{-1} \circ(\mathrm{id} \otimes \Delta)=\left(\mathrm{id} \otimes_{B} \delta^{A}\right) \circ \chi^{-1}$. This identity, restricted to $1 \otimes H$, implies the following property of the translation map:

$$
\left(\mathrm{id} \otimes_{B} \delta^{A}\right) \circ \tau=(\tau \otimes \mathrm{id}) \circ \Delta .
$$

That is, $\tau: H \rightarrow A \otimes_{B} A$ is an $H$-comodule map for $H$ with coaction given by $\Delta$ (that is the coaction in (A.2) restricted to $1 \otimes H$ ) and $A \otimes_{B} A$ with coaction as in (A.2).

Similarly, the condition for $\chi^{-1}$ to be a morphism in $\mathcal{M}^{H}$ with respect to the $H$ coactions in (A.3) gives

$$
\left[(\mathrm{id} \otimes \mathrm{flip}) \circ\left(\delta^{A} \otimes_{B} \mathrm{id}\right)\right] \circ \tau=(\tau \otimes \mathrm{id}) \circ[(\mathrm{id} \otimes S) \circ \text { flip } \circ \Delta] .
$$

Finally, being $\chi_{\left.\right|_{1 \otimes H}}$ a comodule morphism with respect to the $H$-coactions (A.4) and (A.5), one obtains that

$$
\delta^{A \otimes_{B} A} \circ \tau=(\tau \otimes \mathrm{id}) \circ \mathrm{Ad}
$$

\section{Acknowledgments.}

We thank Rita Fioresi, Catherine Meusburger and Alexander Schenkel for fruitful discussions. All authors are members of COST Action MP1405 QSPACE. PA acknowledges support and hospitality from ICTP - INFN during his scientific visit in Trieste, and partial support from INFN, CSN4, Iniziativa Specifica GSS and from INdAM-GNFM. This research has a financial support from Università del Piemonte Orientale. GL acknowledges partial support from INFN, Iniziativa Specifica GAST and from INdAM-GNSAGA. Part of the work of CP was carried out at Université Catholique de Louvain (IRMP) in Louvain-la-neuve. CP gratefully acknowledges support by the Belgian Scientific Policy under IAP grant DYGEST, as well as support by COST (Action MP1405) and INFN Torino during her scientific visits in Torino.

\section{REFERENCES}

[1] P. Aschieri, P. Bieliavsky, C. Pagani, A. Schenkel, Noncommutative Principal Bundles Through Twist Deformation. Commun. Math. Phys. 352 (2017) 287-344.

[2] S. Brain, G. Landi, Moduli spaces of non-commutative instantons: gauging away non-commutative parameters. Q. J. Math. 63 (2012) 41-86.

[3] T. Brzeziński, Translation map in quantum principal bundles. J. Geom. Phys. 20 (1996) 349-370.

[4] T. Brzeziński, S. Majid, Quantum Group Gauge Theory on Quantum Spaces. Commun. Math. Phys. 157 (1993), 591-638.

[5] L. Castellani, Gauge theories of quantum groups. Phys. Lett. B 292 (1992) 93-98.

[6] L. Castellani, Differential calculus on ISOq(N), quantum Poincaré algebra and q-gravity. Comm. Math. Phys. 171 (1995) 383-404.

[7] A. Connes, M.R. Douglas, A.S. Schwarz, Noncommutative geometry and matrix theory: compactification on tori. J. High Energy Phys. 9802 (1998) 003, 35 pages.

[8] A. Connes, M. Dubois-Violette, Noncommutative finite-dimensional manifolds. I. Spherical manifolds and related examples. Commun. Math. Phys. 230 (2002) 539-579.

[9] L. Dąbrowski, H. Grosse, P.M. Hajac, Strong connections and Chern-Connes pairing in the HopfGalois theory. Comm. Math. Phys. 220 (2001) 301-331.

[10] S. Dăscălescu, C. Năstăsescu, S. Raianu, Hopf algebras. An introduction. Monographs and Textbooks in Pure and Applied Mathematics, 235. Marcel Dekker (2001).

[11] Y. Doi, Braided bialgebras and quadratic bialgebras. Comm. Algebra 21 (1993) 1731-1749.

[12] V.G. Drinfeld, On constant quasiclassical solutions of the Yang-Baxter quantum equation. Soviet Math. Dokl. 28 (1983) 667-671. 
[13] M. Durdevic, Quantum gauge transformations and braided structure on quantum principal bundles. arXiv:q-alg/9605010.

[14] V.G. Drinfeld, Hopf algebras and the quantum Yang-Baxter equation. Soviet Math. Dokl. 32 (1985) $254-258$.

[15] M. Dubois-Violette, G. Landi, Noncommutative products of Euclidean spaces. Lett. Math. Phys. 108 (2018) 2491-2513.

[16] L.D. Faddeev, N.Yu. Reshetikhin, L.A. Takhtadzhyan, Quantization of Lie groups and Lie algebras. Leningrad Math. J. 1 (1990)193-225.

[17] D. Husemoller, Fibre bundles. Third edition. Graduate Texts in Mathematics, 20. Springer, 1994.

[18] B. Jurco, S. Schraml, P. Schupp, J. Wess, Enveloping algebra-valued gauge transformations for nonabelian gauge groups on non-commutative spaces. Eur. Phys. J. C Part. Fields 17 (2000) 521-526.

[19] C. Kassel Principal fiber bundles in non-commutative geometry. Quantization, geometry and noncommutative structures in mathematics and physics, Math. Phys. Stud., Springer 2017, pp. 75-133.

[20] A. Klimyk, K. Schmüdgen, Quantum groups and their representations. Springer, 1997.

[21] G. Landi, W.D. Van Suijlekom, Principal fibrations from noncommutative spheres. Commun. Math. Phys. 260 (2005) 203-225.

[22] G. Landi, W.D. Van Suijlekom, Noncommutative instantons from twisted conformal symmetries. Comm. Math. Phys. 271 (2007) 591-634.

[23] S. Majid, Algebras and Hopf algebras in braided categories. Advances in Hopf Algebras. Lec. Notes Pure and Applied Maths 158 (1994) pp. 55-105.

[24] S. Majid, Foundations of quantum group theory. CUP, 1995.

[25] C. Meusburger, D.K. Wise, Hopf algebra gauge theory on a ribbon graph. arXiv:1512.03966.

[26] S. Montgomery, Hopf algebras and their actions on rings. AMS 1993.

[27] N.Yu. Reshetikhin, Multiparameter Quantum Groups and Twisted Quasitriangular Hopf Algebras. Lett. Math. Phys. 20 (1990) 331-335.

[28] A. Schirrmacher, Multiparameter R-matrices and their quantum groups. J. Phys. A: Math. Gen. 24 (1991) L1249-L1258.

[29] H.-J. Schneider, Representation theory of Hopf-Galois extensions. Israel J Math. 72 (1990) $196-231$.

[30] N. Seiberg, E. Witten, String theory and noncommutative geometry. J. High Energy Phys. 9909 (1999) 032, 93 pages.

[31] S.L. Woronowicz, Differential calculus on compact matrix pseudogroups (quantum groups). Comm. Math. Phys. 122 (1989) 125-170.

Paolo Aschieri

Università del Piemonte Orientale,

Dipartimento di Scienze e Innovazione Tecnologica

Viale T. Michel 11, 15121 Alessandria, Italy,

AND INFN Torino, via P. Giuria 1, 10125 Torino, Italy,

and Arnold-Regge Centre, Torino, via P. Giuria 1, 10125 Torino, Italy

E-mail address: paolo.aschieri@uniupo.it

Giovanni Landi

Matematica, Università di Trieste,

Via A. Valerio, 12/1, 34127 Trieste, Italy,

Institute for Geometry and Physics (IGAP) Trieste, Italy

AND INFN, TRIESTE, ITALY

E-mail address: landi@units.it

Chiara Pagani

Università del Piemonte Orientale,

Dipartimento di Scienze e Innovazione Tecnologica

Viale T. Michel 11, 15121 Alessandria, ItAly

AND INDAM-GNSAGA

E-mail address: chiara.pagani@uniupo.it 